\title{
WestVirginiaUniversity
}

THE RESEARCH REPOSITORY @ WVU

Graduate Theses, Dissertations, and Problem Reports

2010

\section{Measuring the Discriminative Capability of Metrology in Human Recognition}

\author{
Rakesh Sepuri \\ West Virginia University
}

Follow this and additional works at: https://researchrepository.wvu.edu/etd

\section{Recommended Citation}

Sepuri, Rakesh, "Measuring the Discriminative Capability of Metrology in Human Recognition" (2010). Graduate Theses, Dissertations, and Problem Reports. 4654.

https://researchrepository.wvu.edu/etd/4654

This Thesis is protected by copyright and/or related rights. It has been brought to you by the The Research Repository @ WVU with permission from the rights-holder(s). You are free to use this Thesis in any way that is permitted by the copyright and related rights legislation that applies to your use. For other uses you must obtain permission from the rights-holder(s) directly, unless additional rights are indicated by a Creative Commons license in the record and/ or on the work itself. This Thesis has been accepted for inclusion in WVU Graduate Theses, Dissertations, and Problem Reports collection by an authorized administrator of The Research Repository @ WVU. For more information, please contact researchrepository@mail.wvu.edu. 


\section{Measuring the Discriminative Capability of \\ Metrology in Human Recognition}

by

\section{Rakesh Sepuri}

Thesis submitted to the College of Engineering and Mineral Resources at West Virginia University

in partial fulfillment of the requirements

for the degree of

Master of Science

in

Electrical Engineering (Communications \& Signal Processing)

Approved by

Dr. Donald Adjeroh, Committee Chairperson

Dr. Arun Ross

Dr. Xin Li

Lane Department of Computer Science and Electrical Engineering

Morgantown, West Virginia

2010

Keywords: Metrology, CAESAR dataset, biometric system, feature extraction, soft biometrics, statistical analysis, performance measurement 


\section{ABSTRACT \\ Measuring the Discriminative Capability of Metrology in Human Recognition \\ By Rakesh Sepuri}

Biometrics is now a more established and sophisticated field. It studies the use of physiological characteristics or behavioral traits to identify and recognize a person automatically. It facilitates theft control and increased security.

Metrology has been one of the well-studied topics in computer vision. Absolute measurement values of humans can be obtained from a fully calibrated camera. These measurements are stored as a database and studied in detail to assess their significance as a biometric. In this thesis we want to assess the performance of human body measurements as a soft biometric. Every human has distinct biometric characters. They can be classified using biometric measurements.

Here the performance of Biometric systems is measured empirically without explicitly measuring the available information. We make use of soft biometric traits like height, weight, gender, age to measure the discriminative capability of metrology in human recognition. Analysis of human body measurements can be applied in various domains like video surveillance, video retrieval, human-computer interaction systems, and medical diagnosis. We establish the performance of human metrology in distinguishing between humans using a database of such measurements. We characterize the performance using measures such as distance plots, precision and recall, genuine acceptance rate (GAR), and false acceptance rate (FAR). 


\section{DEDICATION}

I dedicate this thesis to my parents and brother

Ravi Kumar Sepuri

Susheela Sepuri

Sumesh Sepuri 


\section{ACKNOWLEDGMENTS}

This work would not have been complete without the guidance and help of several individuals who directly or indirectly have contributed and extended their valuable assistance in the completion of this thesis.

First and foremost, I would like to take the opportunity to thank my advisor and committee head Dr. Donald Adjeroh for his valuable guidance and advice. He supported me throughout my work with his timely suggestions and patience. He inspired me greatly in working through this project. His ideas are innovative, and his problem solving skills are commendable. He has always motivated me to work with zeal in my research work. I feel highly honored to work under him and making my Masters program a memorable experience.

I would also like to thank Dr. Arun Ross under whom I learnt the course Pattern Recognition, which partly forms a basis for this work. His lectures were very helpful in doing my ground work. His command over the subject of pattern recognition is supreme. I am very grateful to $\mathrm{Dr}$. Xin $\mathrm{Li}$ too for being on my committee.

Also, I would like to thank my family members, friends and well wishers for their understanding and support. They were always cordial and encouraged me in my work, providing a conducive environment to carry out this work.

Last but not the least, I give thanks to the Almighty Lord for answering my prayers and giving me strength in difficult times. 


\section{TABLE OF CONTENTS}

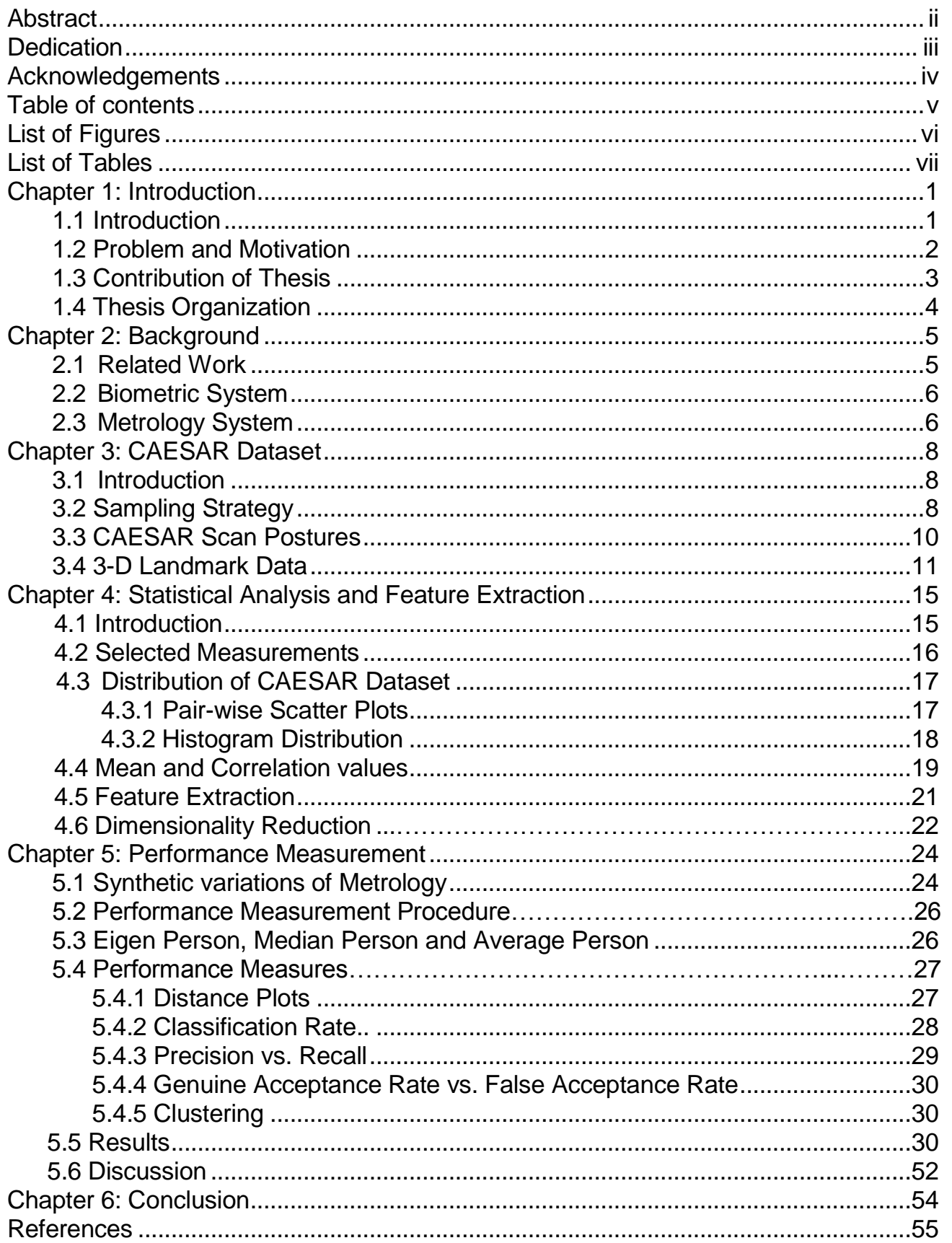




\section{LIST OF FIGURES}

Figure 1: N Visual Index of 3-D Landmarks in Pose A, Upper \& Lower Body, Front View ............12

Figure 2: Pair-wise scatter plots .......................................................................................... 18

Figure 3: Histogram distribution of each measurement over entire population ............................. 19

Figure 4: Variability of distance over entire population with number of variations $\mathrm{N}$.....................12

Figure 5: Relation between different error rates and their dependence on threshold .....................29

Figure 6: Average person-to-person distance along the population considering originals.............32

Figure 7: Average person-to-person distance among the training set for Case 1 ..........................33

Figure 8: Average person-to-person distance among the training set for Case 2 ..........................34

Figure 9: Average person-to-person distance among the training set for Case 3 .........................35

Figure 10: Classification rate as a function of threshold for originals ..........................................36

Figure 11: Classification rate as a function of threshold for each case ...........................................38

Figure 12: Classification rates for each age group considering male and female genders..............40

Figure 13: Precision vs. Recall curve for both training and complete set ....................................42

Figure 14: Genuine Acceptance Rate vs. False Acceptance Rate ..............................................43

Figure 15: Probability of occurrence of Genuine and Imposters considering 44 measurements.....45

Figure 16: Probability of occurrence of Genuine and Imposters considering 88 measurements.....46

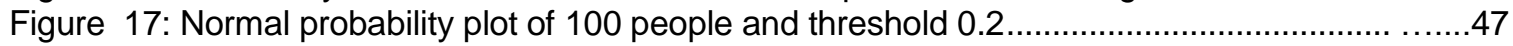

Figure 18: Probability of occurrence of genuine and imposters

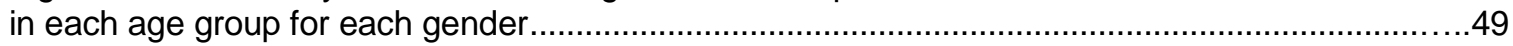

Figure 19: Clustering of training set considering 44 and 88 measurements..................................51 


\section{LIST OF TABLES}

Table 1: Total Number of subjects in each stratum in North America........................9

Table 2: Target Number of subjects in each stratum in The Netherlands ...............9

Table 3: Target Number of subjects in each stratum in Italy ...................................10

Table 4: Landmark Points for measurement and SET-44 measurements

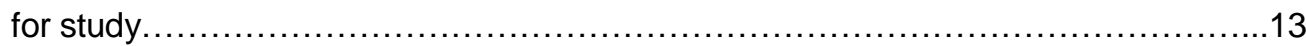

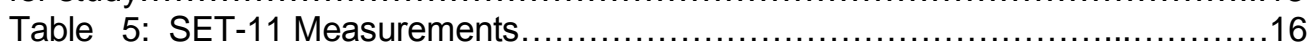

Table 6: Average value in $\mathrm{mm}$ of the SET-11 measurements...........................19

Table 7: Correlation matrix of SET-11 measurements ........................................21

Table 8: Correlation matrix of SET-44 measurements ..........................................21

Table 9: Maximum, Minimum and Average distances of 44

and 88 measurements considering originals..............................................................33

Table 10: Maximum, Minimum and Average distances of 44

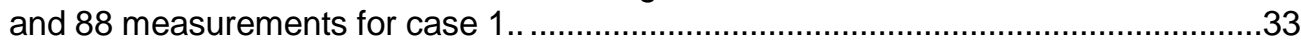

Table 11: Maximum, Minimum and Average distances of 44

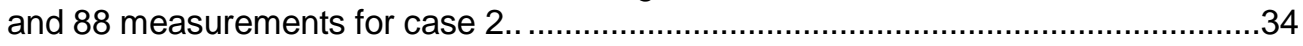

Table 12: Maximum, Minimum and Average distances of 44

and 88 measurements for case 3 . 


\section{Chapter 1: Introduction}

\subsection{Introduction}

We see many kinds of images in our daily life, surrounding us wherever we go. An image can be a photograph or a video frame. Images convey considerable amount of information about the object photographed. Objects can be 2-dimensional, such as photograph or 3-dimensional, like a statue. To be precise, an image is a 2-dimensional snapshot of the object photographed by a camera. It becomes very important to retrieve the information from this image correctly. Taking measurements on the image will not give correct results of the original object. For reliable measurements, it is wise to take measurements on the original objects.

Metrology is the scientific study of measurements. It is the act of assigning a particular value to a physical variable. Measurements provide a basis for opinion on processing information, quality control, and process control. The choice of the measuring device chosen is also important as it depends on resolution and range of desired measurement. A dependable technology nowadays is Video Metrology, performed with video measuring systems. The availability of sophisticated video cameras has thrown more interest on metrology using computer vision techniques.

A camera captures an image, and the desired portion of image is optically magnified. Later the magnified image is converted into a video signal which is studied under various electronics and software methods to determine its features. Reasons why video metrology is prominent are - (i) video contains multiple frames. Hence it provides more reliable results. (ii) It results in effortless automation in video processing. For video metrology, there are two types of cameras in use stationary cameras and planar motion cameras. The current video-based metrology methods are extended from image-based metrology approaches [1]. Image-based metrology has limitations like the algorithms are not open to error analysis and it depends on parallel line segments. On the other hand, video-based metrology composes of multiple frames, hence these limitations are overcome.

An interesting application of metrology is in the study of human feature extraction and measurements. The features are different measurable body parts. Every Human has some dissimilar measurements, even though other measurements are identical. 
The main objective of this work is to examine whether the data provided is reliable in discriminating between people. This can be answered based on performance measures such as Precision \& Recall, and ROC curves. It is critical to know the performance of a system completely before validating the system. The tangible dataset used in this work is made available through CAESAR survey [2].

\subsection{Problem and Motivation}

In recent years, biometric recognition has become more challenging. Biometric recognition plays a vital role in various fields like surveillance and activity monitoring, human computer interaction, intelligent environments, etc. Biometric features can't be borrowed, stolen, or forgotten, and making a false biometric feature is not easily achievable. Some popular methods for biometric recognition include iris scan, fingerprint recognition, face recognition, voice recognition, etc. These methods were extensively studied and used widespread. A brief description of each biometric recognition method can be found in [3]. Some methods related to human metrology are briefed here.

Facial recognition is to identify or verify a person automatically from an image or video frame. This is done by comparing the selected facial features from that person and comparing with facial database. Every face has numerous distinguishable landmarks. These are the different peaks and valleys making up facial features. Some features are distance between the eyes, width of the nose, shape of cheekbones, etc. Each human face consists of around 80 nodal points [4]. All these nodal points are measured resulting in a numerical code known as faceprint [4], which is that person's face in the facial database. This recognition method is non-intrusive and a cheap technology. However it suffers from flaws when there is a lighting change, or person covers his face (mask), or growth of facial hair with age.

Hand Geometry recognition is one of the longest implemented biometric recognition methods. The recognition system constitutes measuring and recording the length, width, thickness, and surface area of the person's hand while placed on a scanner. These systems use a camera to capture a silhouette image of the placed hand. They are a popular choice because of their ease of use, stand-alone capabilities, and less data requirements. It can be easily integrated into other devices/systems. However it has some constraints like the hardware is very expensive, and significant in size. Also some people can't place their hand properly on the scanner.

Gait recognition is to identify a person by recognizing the way that person walks. Much biometric advancement in this field has led to the improvement of gait recognition algorithms. 
Gait has many advantages while acquiring images. The images can be obtained easily from a simple camera, even in public places and without the knowledge of the subject. But it has limitations, like we do not know the degree to which a person's gait is unique. Moreover gait can be affected by factors like footwear, fatigue, terrain, and injury.

Human identification can be done through the above recognition methods. However, the available recognition methods have their respective drawbacks. A common drawback of the human recognition methods is the subject should be willing to make the scans on him/her. This may not be the case always. Sometimes we want to know biometric details of a person without their knowledge. A major factor influencing this kind of identification is the distance of subject from camera. The person under study can be located at any distance from the camera. In such scenarios, video metrology plays a key role in human recognition.

Study of distanced objects from the camera is of profound interest to many researchers. Government and Private Agencies have carried out many related projects in this field before. For example, The Information Processing Technology Office (an agency of DARPA) ran a program known as "Human Identification at a Distance" which developed technologies that are capable of identifying a person at up to $500 \mathrm{ft}$ by their facial features.

Hence it is significant to recognize humans at a distance from the camera, which is commonly observed in the real world. This thesis studies performance of different human measurement features in discriminating between people.

\subsection{Contribution of the Thesis}

As mentioned in the previous human recognition methods, most of these methods have the constraint that the human under study should be within the proximity of the measuring instrument. If this condition isn't satisfied, the system doesn't recognize the human due to erroneous measurements.

The principal aim of this work has been to establish performance of human metrology based on distance plots, precision \& recall, and false acceptance rate \& genuine acceptance rate. We have used the CAESAR database for calculating the performance measurements. From the complete dataset provided, we selected some features of both male \& female subjects which are commonly used, and later feature extraction is performed to achieve uncorrelated dataset.

This thesis details the performance measurement through some ROC curves. Also some characteristic plots are shown to augment the performance of this database. 


\subsection{Thesis Organization}

Chapter 2 is a brief overview of studies carried out before in the field of human metrology. Related research dealing with CAESAR data or statistical analysis is briefed. Also biometric system is explained in brief, along with function of metrology system.

Chapter 3 discusses about CAESAR dataset in detail. This chapter gives a description of the survey and the data collected and produced, along with the samples strategy. Later the CAESAR scan postures are illustrated along with 3-D Landmark data points.

Chapter 4 deals with statistical analysis, the variation of CAESAR data among the population. Later we study about the different pattern recognition methods we made use in this work. This covers classification models, clustering, feature extraction and feature selection.

Chapter 5 is devoted to the performance measurement of CAESAR data. Here we implement the algorithm for this measurement. Later the performance measures are discussed in detail along with the plots.

Chapter 6 concludes our study with suggestions for future directions. 


\section{Chapter 2: Background}

\subsection{Related Work}

Object and feature identification has been an active area in machine vision learning for the past two decades. Among this, few studies focused on identification and analysis of human body motions captured by video images [5]. In 1984, Akita [6] focused on coarse recognition tasks of human body parts. In their study, each sub program which are entitled as body parts (head, legs, arms, etc), corresponds to the labeled binary output image representing different parts of the body. But most of the previous related techniques were utilizing mono imagery for recovery of non-metrical information from images.

In the recent past, Researchers have generated interest in analyzing human body shapes, their reconstruction, and search for a reliable way to cluster humans. CAESAR dataset has been used extensively before, depending on the requirements. For example, Allen et al. described a template-based reconstruction strategy to establish correspondence among same structures, but which have significant deviation in shape [7]. They formulated an objective function to trade off the fit within the data, and fit high resolution template meshes for human body scans using sparse 3D markers. They demonstrated that the scans could be matched to reasonable degree. But a major drawback of their work is the pose of the template should be similar to the target position.

Also, based on user's specifications and need, it is possible to synthesize 3D human body shapes [8]. This is done using a corpus of complete 3D human body laser range scans of different people. A common template mesh is warped on each scanned image, leading to a vertex correspondence between body shapes. Later the variation of body shapes is related with tangible parameters. It also deals with generating human character models automatically.

Godil et al [9] cited static anthropometric distances to simulate computer vision identification biometric system for human identification. They designed a biometric composed of distances among rigid body parts. The advantage of this design is it is invariant to body posture.

Another effort was put in to explain a framework to similarity based retrieval and clustering from a 3D human database [10]. Four methods were developed for searching human database based on similarity of human body and head shape. These head and body descriptors fairly represent the CAESAR bodies accurately. 


\subsection{Biometric System}

A biometric system is a pattern recognition system which recognizes a person based on a feature vector derived from specific intrinsic physical or behavioral characteristics possessed by that person. After the features are extracted, they are stored in a database. Biometric characteristics are of two types:

- Physiological traits which are related to shape of the body, like DNA, fingerprint, face recognition, iris recognition, odor, etc.

- Behavioral traits which mean the behavior of a person, like gait, voice, keystroke, signature, etc.

In terms of reliability, a biometric system based on physiological characteristics is more preferred compared to a biometric system adopted on behavioral characteristics. A biometric system operates on two modes [2]:

- Verification mode where system captures a biometric data of the person and compares with original stored templates of that person in the system database. This is done to verify that the individual is who he claims to be. This is generally done using a smartcard, PIN, username or ID number.

- Identification mode where the captured biometric data of a person is compared against templates corresponding to all users. This is done to identify an unknown individual. The identification of unknown person takes place only if the comparison is within a set threshold.

A human characteristic can be used for recognition through a biometric system in terms of some parameters. Biometric systems must be continuously evaluated and verified in order to be accepted. Biometrics is the core component in a video metrology system. The basic functionality of a biometric system is retained in human recognition through video measurement.

\subsection{Metrology system}

As mentioned before metrology is the science of measurement. It includes all theoretical and practical aspects of measurement. It is always good to get correct measurements, because wrong or inaccurate measurements can lead to wrong decisions. This can have serious consequences, costing money and sometimes lives. It is important to have reliable and accurate measurements approved by relevant authorities worldwide. Also the ever increasing demand for greater accuracy and increased reliability should be satisfied. 
An important concept in metrology is traceability. It is the property of the result of a measurement which can be related to stated references. The level of traceability establishes the level of compatibility. Traceability is obtained by calibration, thereby establishing a relation between the measuring instrument and measurement standard. Calibration is the process where metrology is applied to measuring equipment and ensures conformity with a known standard of measurement. These standards are coordinated by National Laboratories.

Metrology as a science of measurement attempts to validate the data obtained from test equipment. Practically, it is the verification and validation of predefined standards for these purposes:

- Accuracy - it is the degree to which final product corresponds to measurement standard

- Precision - is ability of a measurement to be reproduced consistently

- Reliability - is consistency of accurate results over consecutive measurements

- Traceability - it is validation that measurement of product conforms to some standards

Accuracy is critical in determining whether a system meets desired requirements. The way system responds can be characterized by two error statistics: False Accept Rate (FAR) and False Reject Rate (FRR).

False Accept Rate is the percentage of imposters accepted, and False Reject Rate is percentage of authorized users rejected. They will be discussed in detail in Chapter 5 of the thesis. 


\section{Chapter 3: CAESAR Dataset}

\subsection{Introduction}

This chapter gives a general description of the survey and the data collected and produced. This data is the principal source of input for this work. Based on some features extracted from these measurements, we determine the performance of human metrology. Materials for this chapter are taken from [11]. More details are provided there.

The Civilian American and European Surface Anthropometry Resource (CAESAR) project was a survey of the civilian populations of three countries representing the North Atlantic Treaty Organization (NATO) countries - The United States of America (USA), The Netherlands, and Italy. The Survey was carried out by US Air Force in alliance with other organizations. The Civilian population of these three countries was sampled in an effort to characterize the population of NATO countries as a whole. The main reason for choosing these populations is because of the diversity the three countries offer - United States has the largest and most diverse population, Netherlands has the tallest population, and Italy has one of the shortest populations.

Typically, the principal product from an anthropometric survey is a document with summary of population statistics, often included by means, standard deviations and percentiles. But for engineering applications just this information is not sufficient. In practical applications we need 3-D data which cannot be summarized from means, standard deviations and percentiles. To satisfy these requirements, CAESAR project was introduced. The outcome is the raw data, including for the first time ever complete 3-D models of all subjects. Thus the product of CAESAR survey was to characterize anthropometric variability of populations, including complete 3-D models of all the subjects.

\subsection{Sampling Strategy}

The populations were sampled by age, race, and gender. The reason for using age as strata was to ensure that all racial groups' body sizes and shapes are adequately represented. A stratified sampling plan was used with equal sample size in each cell according to ISO recommendations. There are total 42 sampling cells; 18 in North America, 12 in The Netherlands, and 12 in Italy. The minimum sample size for each cell was calculated based on

the following formula:

$$
\frac{|\bar{X}-v| * \sqrt{n_{i}}}{\sigma}=\zeta
$$


where $\zeta=$ eccentricity, $\sigma=$ standard deviation, $n_{i}=$ sample size, $v=$ true mean of the group, $\bar{X}=$ sample mean of the group, and $|\bar{X}-v|=$ desired within cell accuracy.

The total number target for sample in one country was sum of sample sizes in subgroups. Stature measurement was used to estimate the sample size of that subject. This is because stature measure gives the most conservative estimate. A reasonable within cell standard deviation estimate for stature is $70 \mathrm{~mm}$. The desired within cell accuracy was set to $10 \mathrm{~mm}$.

The calculation of within cell sample size now becomes:

$$
\frac{|10| * \sqrt{n_{i}}}{70}=1.96
$$

Which results in $n_{i}=188$. This value is set as target number of subjects per cell. It is the number to provide a sample mean value that is within $10 \mathrm{~mm}$ of true population mean with a confidence of $95 \%$. The following tables show number of subjects studied in each country for different age groups and races.

\begin{tabular}{|l||l|l|l|l||l|l|l|l|l|}
\hline \multicolumn{1}{|c|}{} & \multicolumn{5}{c||}{ Female } & \multicolumn{3}{c|}{ Male } \\
\hline Age & $18-29$ & $30-44$ & $45-65$ & Sum & & $18-29$ & $30-44$ & $45-65$ & Sum \\
\hline White & 188 & 373 & 394 & 955 & & 191 & 353 & 320 & 864 \\
\hline Black & 61 & 48 & 38 & 147 & & 39 & 52 & 25 & 116 \\
\hline Other & 58 & 56 & 37 & 151 & & 51 & 56 & 30 & 137 \\
\hline Sum & 307 & 477 & 469 & 1253 & & 281 & 461 & 375 & 1117 \\
\hline Total Sum & & & & & 2370 & & & & \\
\hline
\end{tabular}

Table 1: Total Number of subjects in each stratum in North America

\begin{tabular}{|l||l|l|l|l||l||l|l|l|l|}
\hline \multicolumn{4}{|c||}{} & \multicolumn{4}{c|}{ Female } & \multicolumn{4}{c|}{ Male } \\
\hline Age & $18-29$ & $30-44$ & $45-65$ & Sum & & $18-29$ & $30-44$ & $45-65$ & Sum \\
\hline Dutch & 167 & 200 & 177 & 544 & & 156 & 152 & 172 & 480 \\
\hline Other & 41 & 48 & 58 & 147 & & 29 & 23 & 32 & 84 \\
\hline Sum & 208 & 248 & 235 & 691 & & 185 & 175 & 204 & 564 \\
\hline Total Sum & & & & & 1255 & & & & \\
\hline
\end{tabular}

Table 2: Target Number of subjects in each stratum in The Netherlands 


\begin{tabular}{|l||l|l|l|l||l||l|l|l|l|}
\hline \multicolumn{4}{|c||}{} & \multicolumn{4}{c|}{ Female } & \multicolumn{4}{c|}{ Male } \\
\hline Age & $18-29$ & $30-44$ & $45-65$ & Sum & & $18-29$ & $30-44$ & $45-65$ & Sum \\
\hline Italian & 252 & 67 & 57 & 376 & & 235 & 103 & 50 & 388 \\
\hline Other & 5 & 4 & 1 & 10 & & 14 & 7 & 1 & 22 \\
\hline Sum & 257 & 71 & 56 & 386 & & 249 & 110 & 51 & 410 \\
\hline Total Sum & & & & & 796 & & & & \\
\hline
\end{tabular}

Table 3: Target Number of subjects in each stratum in Italy

Now we get the total number of subjects in CAESAR dataset from three countries including both genders as:

North America $=2370 ;$ The Netherlands $=1255 ;$ Italy $=796 ;$ Total $=4431$

In North America, 12 locations were shortlisted for data collection. These locations were selected in proportion to distribution of population in each of 4 regions during that time.

\subsection{CAESAR Scan Postures}

The populations were sampled by age, race, and gender. The products from CAESAR survey consisted of raw files and documentation like demographic data of each subject, 3-D models and landmarks in different postures for each subject, any text files with notes on subject anomalies, summary reports, etc.

Each subject was scanned in three different postures. Pose A is a standing posture. Pose B is a seated posture where the subject assumes a "comfortable working posture". Pose $C$ is another seated posture in which the subject raises his/her arms and head to provide the greatest possible scan coverage.

From these poses, different measurements are obtained. Traditional-style dimensions are measured on right side of body for following body parts: shoulder, ankle, arm, buttock, elbow, eye, foot, hand, knee, thigh, wrist, and scapular and triceps skin folds. For all the measurements, the investigator makes sure the subject is suitably positioned to get an accurate reading. 
There are other measurements taken from 3-D scans. The 3-D scans were processed to combine the information from different scan head within a scan into one object. This results in a complete model for each pose. High-resolution measurements of body surfaces were made using a new data collection technology - 3D surface anthropometry. This technology captures thousands of points in few seconds. It provides details about the surface shape as well as 3D locations of measurements relative to each other. The resulting scan is independent of the person, making it convenient to standardize.

\subsection{3-D Landmark Data}

Before scanning, the subject's body is marked with 72 Anthropometry landmarks using stickers for later identification. 12 stickers were 3-D stickers which are truncated square pyramids in shape. The rest were white circular paper stickers, $12 \mathrm{~mm}$ in diameter. In each pose (A, B, and C), the body was marked with these stickers, as shown in Figure 1, before scanning.

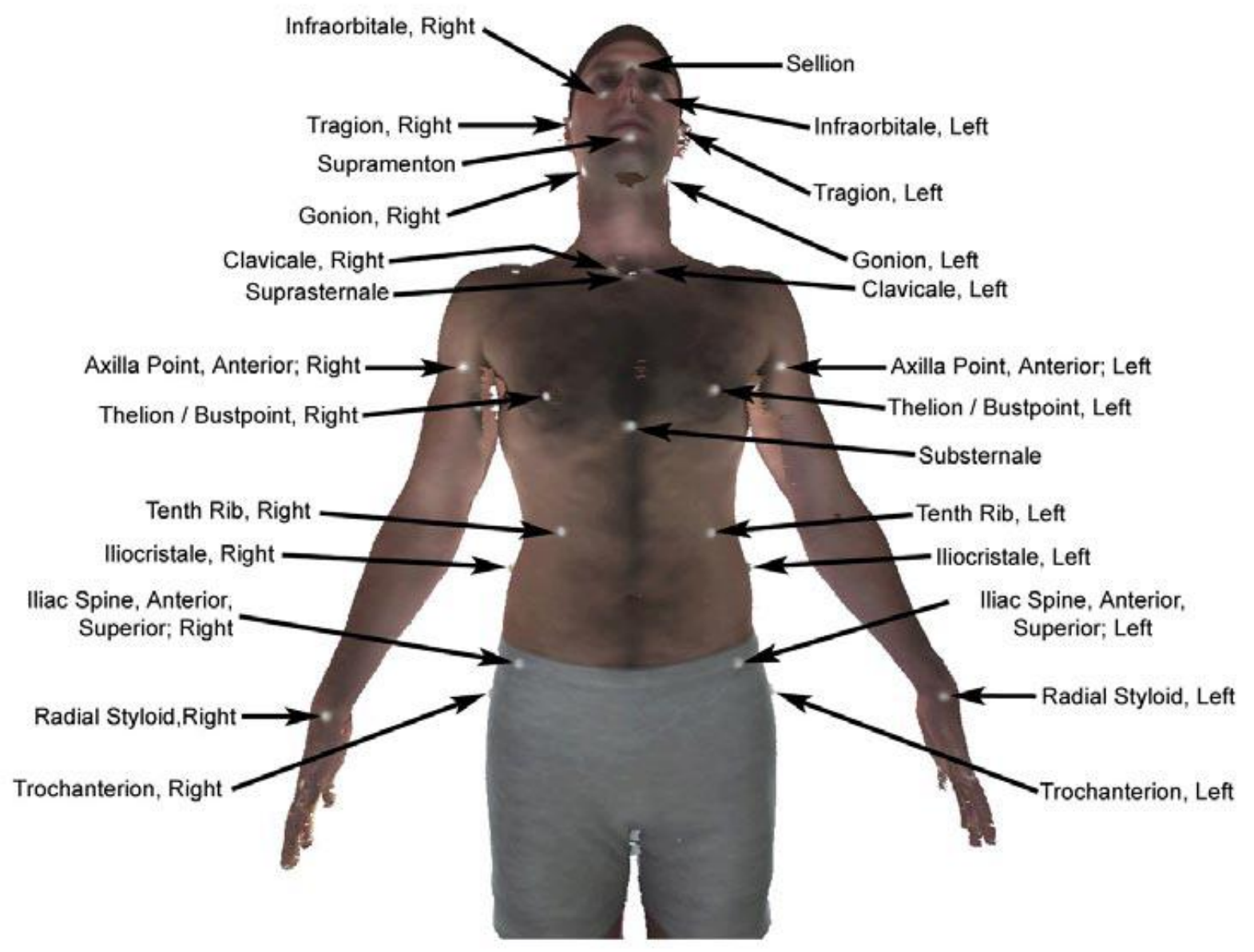

Figure 1a: Visual Index of 3-D Landmarks in Pose A, Upper Body, Front View 

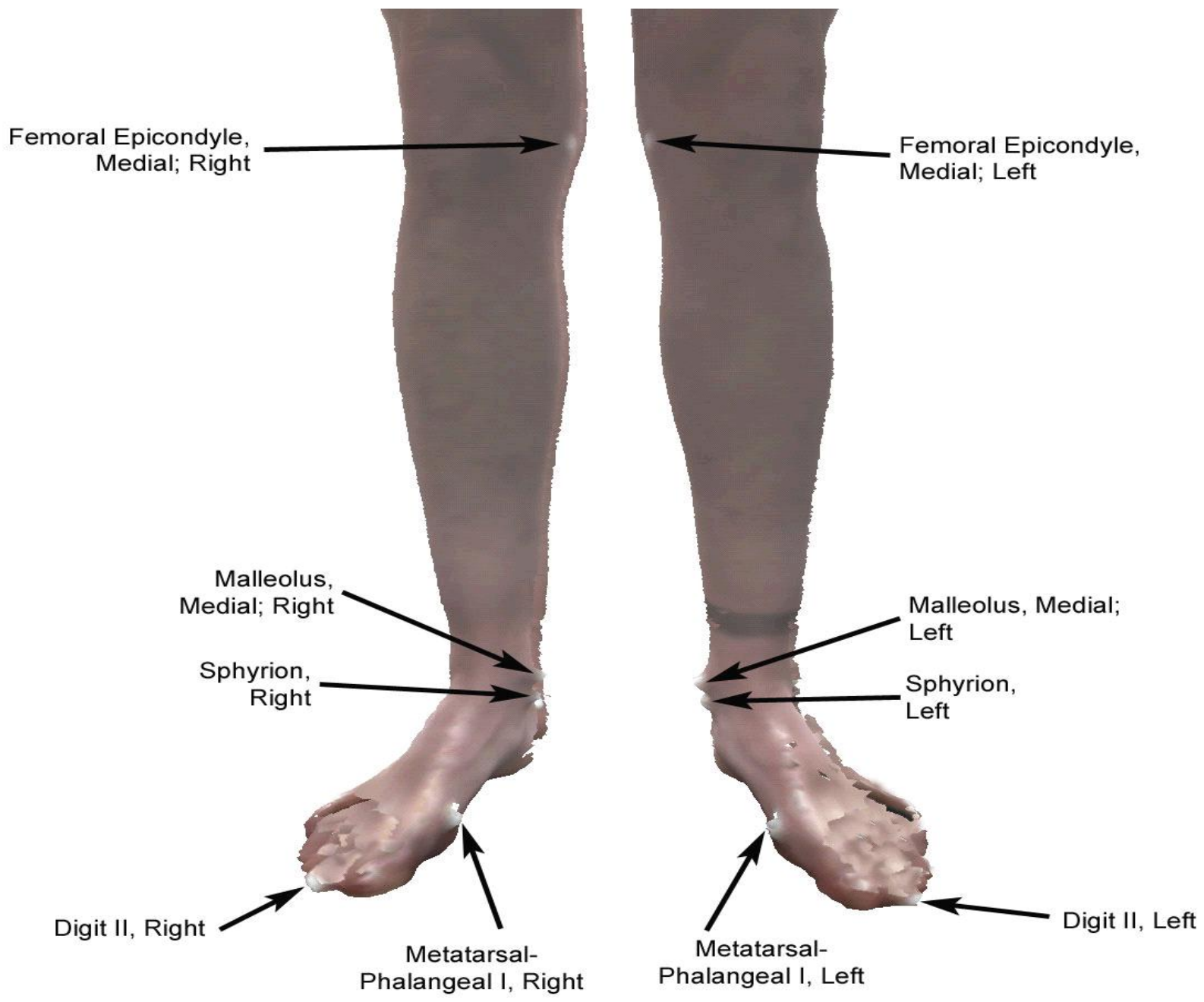

Figure 1b: Visual Index of 3-D Landmarks in Pose A, Lower Body, Front View

The Complete Landmark points for measurement in CAESAR dataset is shown in Table 4a [8]. Table 4b lists 44 measurements we have considered for this study. 
CAESAR Dataset

\begin{tabular}{|c|c|}
\hline $\begin{array}{l}\text { 1. Sellion } \\
\text { 2. Rt. Infraorbitale } \\
\text { 3. Lt. Infraorbitale } \\
\text { 4. Supramenton } \\
\text { 5. Rt. Tragion } \\
\text { 6. Rt. Gonion } \\
\text { 7. Lt. Tragion } \\
\text { 8. Lt. Gonion } \\
\text { 9. Nuchale } \\
\text { 10. Rt. Calvicale } \\
\text { 11. Suprasternale } \\
\text { 12. Lt. Calvicale } \\
\text { 13. Rt. } \\
\text { Thelion/Bustpoint } \\
\text { 14. Lt. } \\
\text { Thelion/Bustpoint } \\
\text { 15. Substernale } \\
\text { 16. Rt. 10 } \\
\text { 17. Rt. ASIb } \\
\text { 18. Lt. } 10^{\text {th }} \text { Rib } \\
\text { 19. Lt. ASIS } \\
\text { 20. Rt. Iliocristale } \\
\text { 21. Rt. } \\
\text { Trochanterion } \\
\text { 22. Lt. Iliocristale } \\
\text { 23. Lt. } \\
\text { Trochanterion } \\
\text { 24. Cervicale } \\
\text { 25. 10 Rib } \\
\text { Midspine } \\
\text { 26. Rt. PSIS } \\
\text { 27. Lt. PSIS } \\
\text { 28. Waist, } \\
\text { Preferred, Post } \\
\text { 29. Rt. Acromion } \\
\text { 30. Rt. Axilla, Ant } \\
\text { 31. Rt. Radial } \\
\text { Styloid } \\
\text { 32. Rt. Axilla, Post } \\
\text { 33. Rt. Olecranon } \\
\text { 34. Rt. Humeral } \\
\text { Lateral Epicn } \\
\text { 35. Rt. Humeral } \\
\text { Medial Epicn } \\
\text { 36. Rt. Radiale } \\
\text { 37. Rt. } \\
\text { Metacarpal-Phal. } \\
\text { II } \\
\end{array}$ & $\begin{array}{l}\text { 38. Rt. Dactylion } \\
\text { 39. Rt. Ulnar Styloid } \\
\text { 40. Rt. Metacarpal-Phal. } \\
\text { V } \\
\text { 41. Lt. Acromion } \\
\text { 42. Lt. Axilla, Ant } \\
\text { 43. Lt. Radial Styloid } \\
\text { 44. Lt. Axilla, Post } \\
\text { 45. Lt. Olecranon } \\
\text { 46. Lt. Humeral Lateral } \\
\text { Epicn } \\
\text { 47. Lt. Humeral Medial } \\
\text { Epicn } \\
\text { 48. Lt. Radiale } \\
\text { 49. Lt. Metacarpal-Phal. } \\
\text { II } \\
\text { 50. Lt. Dactylion } \\
\text { 51. Lt. Ulnar Styloid } \\
\text { 52. Lt. Metacarpal-Phal. } \\
\text { V } \\
\text { 53. Rt. Knee Crease } \\
\text { 54. Rt. Femoral Lateral } \\
\text { Epicn } \\
\text { 55. Rt. Femoral Medial } \\
\text { Epicn } \\
\text { 56. Rt. Metatarsal-Phal. } \\
\text { V } \\
\text { 57. Rt. Lateral Malleolus } \\
\text { 58. Rt. Medial } \\
\text { Malleoulus } \\
\text { 59. Rt. Sphyrion } \\
\text { 60. Rt. Metatarsal-Phal. I } \\
\text { 61. Rt. Calcaneous, Post } \\
\text { 62. Rt. Digit II } \\
\text { 63. Lt. Knee Crease } \\
\text { 64. Lt. Femoral Lateral } \\
\text { Epicn } \\
\text { 65. Lt. Femoral Medial } \\
\text { Epicn } \\
\text { 66. Lt. Metatarsal-Phal. } \\
\text { V } \\
\text { 67. Lt. Lateral Malleolus } \\
\text { 68. Lt. Medial Malleolus } \\
\text { 69. Lt. Sphyrion } \\
\text { 70. Lt. Metatarsal-Phal. I } \\
\text { 71. Lt. Calcaneous, Post } \\
\text { 72. Lt. Digit II } \\
\text { 73. Crotch } \\
\text { 74. Functional Butt Block }\end{array}$ \\
\hline
\end{tabular}

Mean (mm) Std

\begin{tabular}{|c|c|c|}
\hline 1. Acromial Heiaht Sitting & $50<270$ & 27656 \\
\hline 2. Ankle Circumference & 253507 & 21065 \\
\hline 3. Spin-to-Shoulder & 204.407 & 17.208 \\
\hline 4. Spine-to-Elbow & 530.339 & 35.958 \\
\hline 5. Arm Length (Spine to Wrist) & 817.026 & 57.865 \\
\hline 6. Arm Length (Shoulder to Wrist) & 612.618 & 46.005 \\
\hline 7. Arm Length (Shoulder to Elbow) & 325.931 & 24.465 \\
\hline $\begin{array}{l}\text { 8. Armscye Circumference (Scye Circ Over } \\
\text { Acromion) }\end{array}$ & 417.26 & 52.236 \\
\hline 9. Bizygomatic Breadth & 138.502 & 8.207 \\
\hline 10. Chest Circumference & 996.615 & 123.464 \\
\hline 11. Buttock-Knee Length & 602 & 39.969 \\
\hline 12. Chest Girth at Scye (Chest & 984.383 & 117.991 \\
\hline \multicolumn{3}{|l|}{ Circumference at Scye) } \\
\hline 13. Crotch Height & 773.518 & 55.805 \\
\hline 14. Sitting Height & 893.734 & 48.811 \\
\hline *15. Stature & 1704.673 & 102.711 \\
\hline 16. Subscapular Skinfold & 20.747 & 11.103 \\
\hline 17. Thigh Circumference & 607.204 & 69.461 \\
\hline 18. Thigh Circumference Max Sitting & 607.288 & 68.647 \\
\hline 19. Thumb Tip Reach & 774.221 & 56.254 \\
\hline 20. Thumb Tip Reach 1 & 772.582 & 56.685 \\
\hline 21. Thumb Tip Reach 2 & 775.116 & 56.545 \\
\hline 22. Thumb Tip Reach 3 & 775.092 & 56.682 \\
\hline 23. Elbow Height, Sitting & 238.982 & 28.028 \\
\hline 24. Eye Height, Sitting & 780.235 & 45.173 \\
\hline *25. Face Length & 116.530 & 8.537 \\
\hline 26. Foot Length & 253.07 & 19.915 \\
\hline 27. Hand Circumference & 197.71 & 17.685 \\
\hline 28. Hand Length & 192.142 & 14.91 \\
\hline *29. Head Breadth & 150.763 & 7.26 \\
\hline 30. Head Circumference & 564.753 & 21.481 \\
\hline *31. Head Length & 193.888 & 9.480 \\
\hline 32. Hip Breadth, Sitting & 395.957 & 43.966 \\
\hline 33. Hip Circumference, & 1049.87 & 111.802 \\
\hline \multicolumn{3}{|l|}{ Maxim } \\
\hline 34. Hip Circ Max Height & 851.514 & 69.315 \\
\hline${ }^{*} 35$. Knee Height & 534.173 & 39.921 \\
\hline 36. Neck Base & 437.326 & 40.193 \\
\hline \multicolumn{3}{|l|}{ Circumference } \\
\hline *37. Shoulder Breadth & 460.953 & 48.268 \\
\hline 38. Triceps Skinfold & 18.607 & 9.769 \\
\hline 39. Total Crotch Length (Crotch Length) & 676.654 & 71.942 \\
\hline 40. Vertical Trunk & 1645.225 & 129.204 \\
\hline \multicolumn{3}{|l|}{ Circumference } \\
\hline 41. Waist Circumference, Preferred & 847.877 & 143.491 \\
\hline 42. Waist Front Length & 418.649 & 65.603 \\
\hline *43. Waist Height, & 1025.878 & 59.908 \\
\hline Preferred & & \\
\hline 44. Weight & 77.014 & 19.660 \\
\hline
\end{tabular}

Table 4b: 44 measurements selected for this study along with their average values and standard deviations

NOTE: * indicates those in our smaller set of 11 measurements

Table 4a: Landmark Points for measurement 
Based on the landmarks listed in Table 4a, there were total 99 measurements. Since many of the traditional measurements have been used for many years, it is more reliable to take some measurements the traditional way. As a result 40 measurements were taken with calipers and tape measures, and 59 measurements were point to point or point to surface, that are calculated from the scan points. In the latter measurements, 43 were calculated from 3-D landmarks from standing pose and 16 were calculated from 3-D landmarks from seated pose. The measurements are in alphabetical order within each section according to their CAESAR name. The measurements are stored in both English units (inches) and metric units (millimeters). The CAESAR name is consistent with ISO rules. The body part or point is listed first, followed by the type of measurement. This is followed by the pose, if necessary, and later followed by the side of body when both are measured (Left or Right). In the data ISO names are mentioned, along with name used in raw data file provided. This complete data is provided in both ASCII text and EXCEL spreadsheet files.

In case of any missing values in the measurement of a subject, we estimate the expected value of this measurement from this subject's remaining measurements. Here we predict any missing data based on the other measurements of a person. In the complete dataset of 2370 persons, there were 5 persons with few missing measurements. 


\section{Chapter 4: Statistical Analysis and Feature Extraction}

\subsection{Introduction}

Data can be collected from existing sources or can be obtained from observation and experimental studies. The patterns in data can be studied for randomness in the observations and we can draw inferences about the population distribution.

The input data for this work is the CAESAR dataset. We want to look at performance of this dataset in classifying humans. First, we want to answer a few questions like what is the nature of the dataset? What is the relation of dataset in underlying population?

Probability is vital in decision making because it provides a mechanism for measuring, expressing, and analyzing uncertainties associated with the data in future events.

In this work, we approach the study of performance by considering the four-step process in learning from data [12]:

1. Defining the problem

2. Selecting the data

3. Summarizing the data

4. Analyzing the data, interpreting the analyses, and communicating the results.

All the above four steps are equally important. The CAESAR dataset has intrinsic behaviors under varying conditions. Before application of CAESAR dataset in scientific applications, it is important to have a thorough knowledge of the behavior of this data. We want to achieve our goal of performance measurement as:

- We want to summarize the whole data in a shorter form through feature extraction

- Use some basic statistical analysis methods on these features

- Design and implement an algorithm to study variations in these measurements

- Establish performance of this data through ROC curves

Understanding the statistics of a dataset thoroughly means to deal with uncertainty introduced by errors in measurement as well as by other fluctuations. Obviously performance should be of 
even greater value in situations of high precision than in those in which the data are affected by large errors.

In this thesis we will be doing some analysis not only on the original data provided, but also on few variations of these original measurements with the help of varying thresholds. We also predict and forecast some features to get expected features. This is discussed in the further sections of this chapter. It is necessary to carefully plan how many features are needed from the complete dataset, and how are they extracted from the original set. Also, what are these features? These questions are answered in the next section.

\subsection{Selected Measurements}

The data for this work is the 99 measurements obtained from CAESAR survey. From this dataset, we initially select 11 features which are easier to extract automatically, and are more often employed. Later we consider 44 features, and performance measurement is established on both these set of features. We call these sets SET-11 and SET-44 measurements respectively.

The SET-11 measurements selected from the original dataset are listed in Table 5:

\begin{tabular}{|c|c|c|c|c|c|c|c|}
\hline $\begin{array}{c}\text { measurement } \\
\text { name }\end{array}$ & code & $\begin{array}{c}\text { landmark } \\
\text { points }\end{array}$ & ID & $\begin{array}{c}\text { measurement } \\
\text { name }\end{array}$ & code & $\begin{array}{c}\text { landmark } \\
\text { points }\end{array}$ & ID \\
\hline body height & bh & 1 & (i) & face breadth & $\mathrm{fb}$ & $2 \& 9$ & (vii) \\
\hline eye height & eh & $2 \& 3$ & (ii) & face length & $\overline{\mathrm{fl}}$ & $1 \& 4$ & (viii) \\
\hline chest height & ch & $13 \& 14$ & (iii) & head breadth & $\mathrm{hb}$ & $5 \& 7$ & (ix) \\
\hline elbow height & eh & $33 \& 45$ & (iv) & head length & $\overline{\mathrm{hl}}$ & $1 \& 9$ & $(\mathrm{x})$ \\
\hline waist height & wh & 28 & (v) & \multirow{2}{*}{$\begin{array}{l}\text { shoulder } \\
\text { breadth }\end{array}$} & \multirow[t]{2}{*}{$\mathrm{sb}$} & \multirow[t]{2}{*}{$29 \& 41$} & \multirow[t]{2}{*}{ (xi) } \\
\hline knee height & $\mathrm{kh}$ & $55 \& 65$ & (vi) & & & & \\
\hline
\end{tabular}

Table 5: SET-11 measurements

For a more comprehensive study of the performance, we later select SET-44 measurements. These are listed in Table 4b. Some measurements present in SET-11 are also indicated with “*' in this table. 


\subsection{Distribution of Measurements in CAESAR dataset}

For uniformity, each selected feature is normalized using two different methods. They are:

$$
\begin{aligned}
& \text { (i) Normalized value }=\frac{(M-\min (M))}{(\max (M)-\min (M))} \\
& \text { (ii) Normalized value }=\frac{(M-\mu)}{\sigma}
\end{aligned}
$$

where, $M$ is the feature vector, $\mu$ and $\sigma$ are mean and standard deviation of feature vector $M$ respectively, $\min (M)$ and $\max (M)$ are the minimum and maximum values of vector $M$ respectively. Each feature is normalized against its own values.

Before performance analysis, it may be informative to analyze the data at hand. We want to observe distribution of the dataset and discover any related patterns. This can be achieved using scatter plots and histogram distributions.

\subsubsection{Pair-wise Scatter Plots}

Scatter plots are bivariate or trivariate plots of variables against each other. They aid in understanding relationships among the variables of a dataset. A downward sloping scatter plot indicates that as we increase the variable on the horizontal axis, the variable on vertical axis decreases. An analogous statement can be made for upward sloping scatters.

Figure 2 shows the pair wise scatter plots between all SET-11 measurements along with a best-fit trend line (marked in red) for the entire population of 2370 subjects. A total of 110 scatter plots were obtained; 10 for each distribution of one feature against all other features. The horizontal and vertical axes range from 0 to 1 due to normalization using Equation 2a.

The plots were used to get a basic idea of the distribution of these easily extractable features. We can know whether the data is displaying an accelerating, decelerating, or stationary trend. Also a "best-fit" trend line is applied to the data points. The proportion of data points above and below the trend line shows variation from the expected values. The initial step in statistical analysis will be to find the correlation and covariance of the provided 
data. From scatter plots we can see that some features are well correlated, and some are uncorrelated. This shows randomness in measured data.

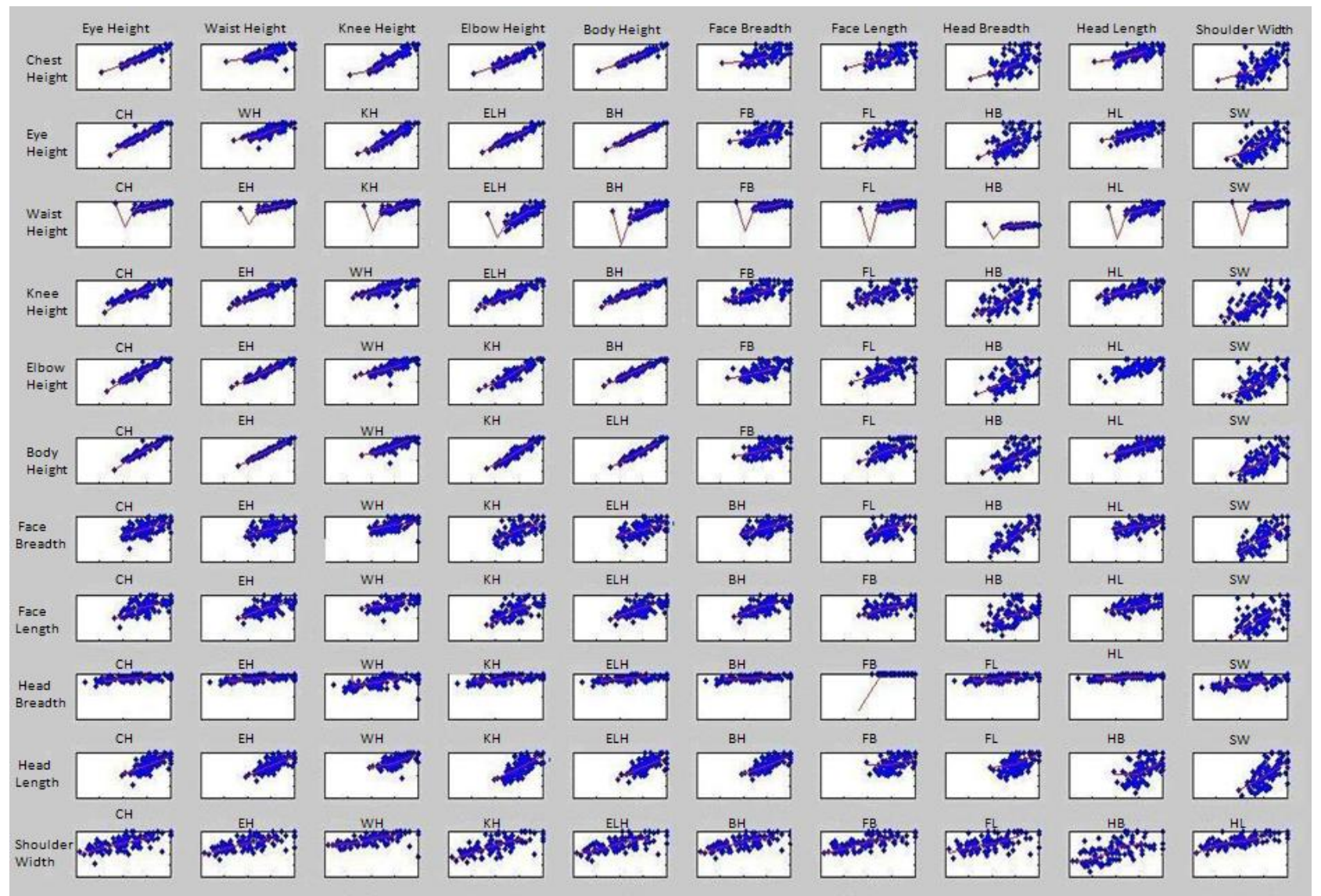

Figure 2: Pair-wise scatter plots for SET-11 measurements over entire population

\subsubsection{Histogram Distribution}

For each measurement, a histogram is also plotted to give more detail about the measurements variation in the population. Figure 3 shows the histogram distribution of SET11 measurements over the entire CAESAR population of $n=2370$ subjects.

From the histogram below, we get an idea of the shape of distribution of this large dataset. They are useful in describing large differences in shape and symmetry. However they can't be used in more precise judgments such as depicting individual values.

Scatter plots and Histograms provide a basic idea of distribution of the measurements in the CAESAR dataset. But to perform further analysis, we have to think of other methods. Visual 

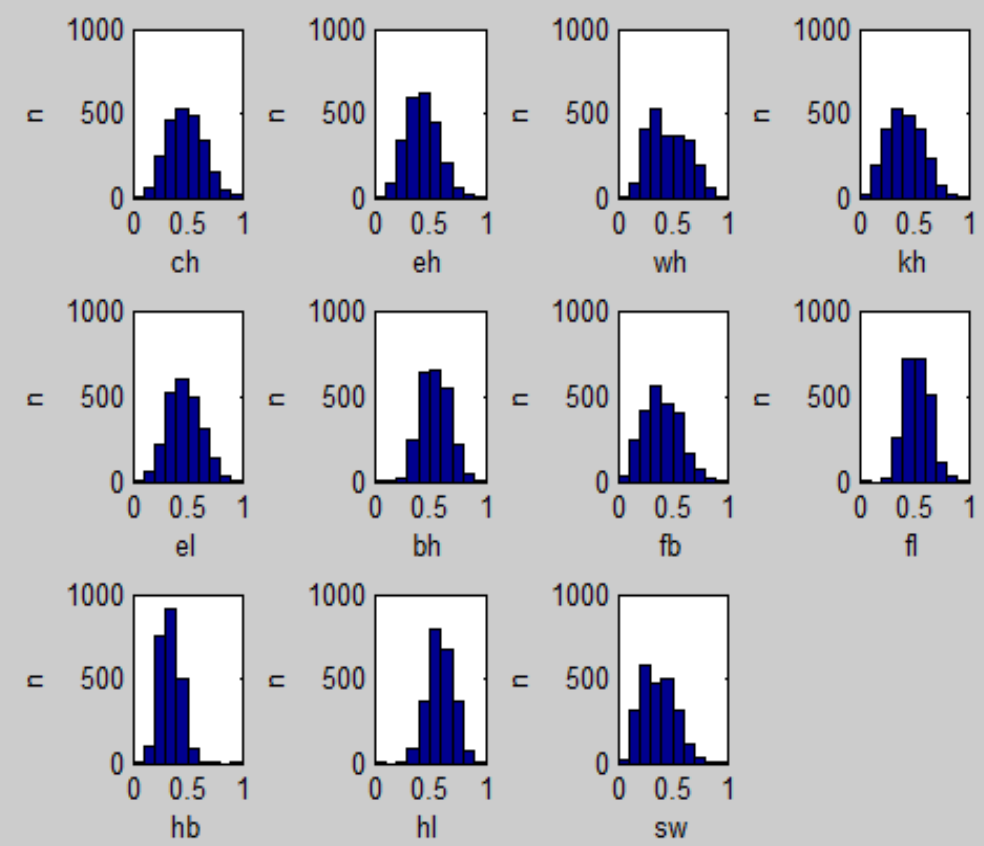

Figure 3: Histogram distribution for SET-11 measurements over entire population

inspection was the method of analysis used for these two plots, along with computation of covariance and correlation of this dataset.

\subsection{Mean and Correlation values}

Over the complete CAESAR data, the average values of the SET-11 features chosen initially are shown in Table 6. The average value of SET-44 features is shown in Table 4b.

\begin{tabular}{|c|c|}
\hline Feature & Average value (mm) \\
\hline Body Height (bh) & 1704.673 \\
\hline Eye Height (eh) & 1470.431 \\
\hline Chest Height (ch) & 1230.884 \\
\hline Elbow Height (el) & 1099.438 \\
\hline Waist Height (wh) & 1025.878 \\
\hline Knee Height (kh) & 534.173 \\
\hline Shoulder Breadth (sb) & 460.953 \\
\hline Face Length (fl) & 116.530 \\
\hline Face Breadth (fb) & 138.43 \\
\hline
\end{tabular}




\begin{tabular}{|c|c|}
\hline Head Length (hl) & 193.888 \\
\hline Head Breadth (hb) & 150.763 \\
\hline
\end{tabular}

Table 6: Average value in $\mathrm{mm}$ of SET-11 measurements

\section{Correlation}

Pearson product-moment correlation coefficient is the most familiar quantity to measure covariance between two variables. The correlation coefficient $\rho_{X, Y}$ between two random variables $X$ and $Y$ is given by

$$
\rho_{X, Y}=\operatorname{corr}(X, Y)=\frac{\operatorname{cov}(X, Y)}{\sigma_{X} \sigma_{Y}}=\frac{E\left[\left(X-\mu_{X}\right)\left(Y-\mu_{Y}\right)\right]}{\sigma_{X} \sigma_{Y}}
$$

where, $\mu_{X} \& \mu_{Y}$ are mean or expected values of variables $X$ and $Y$ respectively, $\sigma_{X} \& \sigma_{Y}$ are corresponding standard deviations of variables $X$ and $Y, E$ is the expected value operator, cov is covariance, and corr is denoted for correlation.

Correlation is a statistical measurement of relationship between two variables. Correlation values range from +1 to -1 . A zero correlation indicates there is no relationship between the features. That means the features are uncorrelated. A correlation value of -1 represents perfect negative (decreasing) linear relationship and a correlation value of +1 represents perfect positive (increasing) linear relationship. Correlations are useful because they indicate a predictive relationship which is useful in understanding about data variation [13].

In MATLAB, correlation is an inbuilt function, denoted by $\operatorname{corr}(X, Y)$ where $X$ and $Y$ can be any of the SET-11 or SET-44 measurements. Function $\operatorname{corr}(X, Y)$ returns a $p_{1}-$-by- $p_{2}$ matrix containing the pair wise correlation coefficient between each pair of columns in the $n-b y-p_{1}$ and $n-b y-p_{2}$ matrices of $X$ and $Y$. The correlation matrices of SET-11 and SET-44 measurements are shown in Table 7 and Table 8 respectively.

\begin{tabular}{|c|c|c|c|c|c|c|c|c|c|c|c|}
\hline & bh & eh & ch & el & wh & kh & sb & fl & fb & hl & hb \\
\hline \hline bh & 1.00 & 0.985 & 0.965 & 0.961 & 0.778 & 0.947 & 0.634 & 0.608 & 0.475 & 0.623 & 0.219 \\
\hline eh & 0.985 & 1.00 & 0.964 & 0.962 & 0.762 & 0.943 & 0.617 & 0.593 & 0.453 & 0.598 & 0.1943 \\
\hline ch & 0.965 & 0.964 & 1.00 & 0.95 & 0.737 & 0.942 & 0.59 & 0.562 & 0.433 & 0.58 & 0.165 \\
\hline el & 0.961 & 0.962 & 0.95 & 1.00 & 0.757 & 0.918 & 0.631 & 0.576 & 0.476 & 0.597 & 0.221 \\
\hline wh & 0.778 & 0.762 & 0.737 & 0.757 & 1.00 & 0.722 & 0.607 & 0.539 & 0.511 & 0.591 & 0.309 \\
\hline
\end{tabular}




\begin{tabular}{|c||c|c|c|c|c|c|c|c|c|c|c|}
\hline $\mathrm{kh}$ & 0.947 & 0.943 & 0.942 & 0.918 & 0.722 & 1.00 & 0.616 & 0.594 & 0.461 & 0.599 & 0.196 \\
\hline \hline $\mathrm{sb}$ & 0.634 & 0.617 & 0.59 & 0.631 & 0.607 & 0.616 & 1.00 & 0.559 & 0.673 & 0.595 & 0.360 \\
\hline \hline $\mathrm{fl}$ & 0.608 & 0.593 & 0.562 & 0.576 & 0.539 & 0.594 & 0.559 & 1.00 & 0.520 & 0.589 & 0.388 \\
\hline $\mathrm{fb}$ & 0.475 & 0.453 & 0.433 & 0.476 & 0.511 & 0.461 & 0.673 & 0.52 & 1.00 & 0.47 & 0.615 \\
\hline $\mathrm{hl}$ & 0.624 & 0.598 & 0.58 & 0.597 & 0.591 & 0.599 & 0.595 & 0.589 & 0.47 & 1.00 & 0.409 \\
\hline $\mathrm{hb}$ & 0.219 & 0.194 & 0.165 & 0.221 & 0.309 & 0.196 & 0.36 & 0.388 & 0.615 & 0.409 & 1.00 \\
\hline
\end{tabular}

Table 7: Correlation matrix of SET-11 measurements

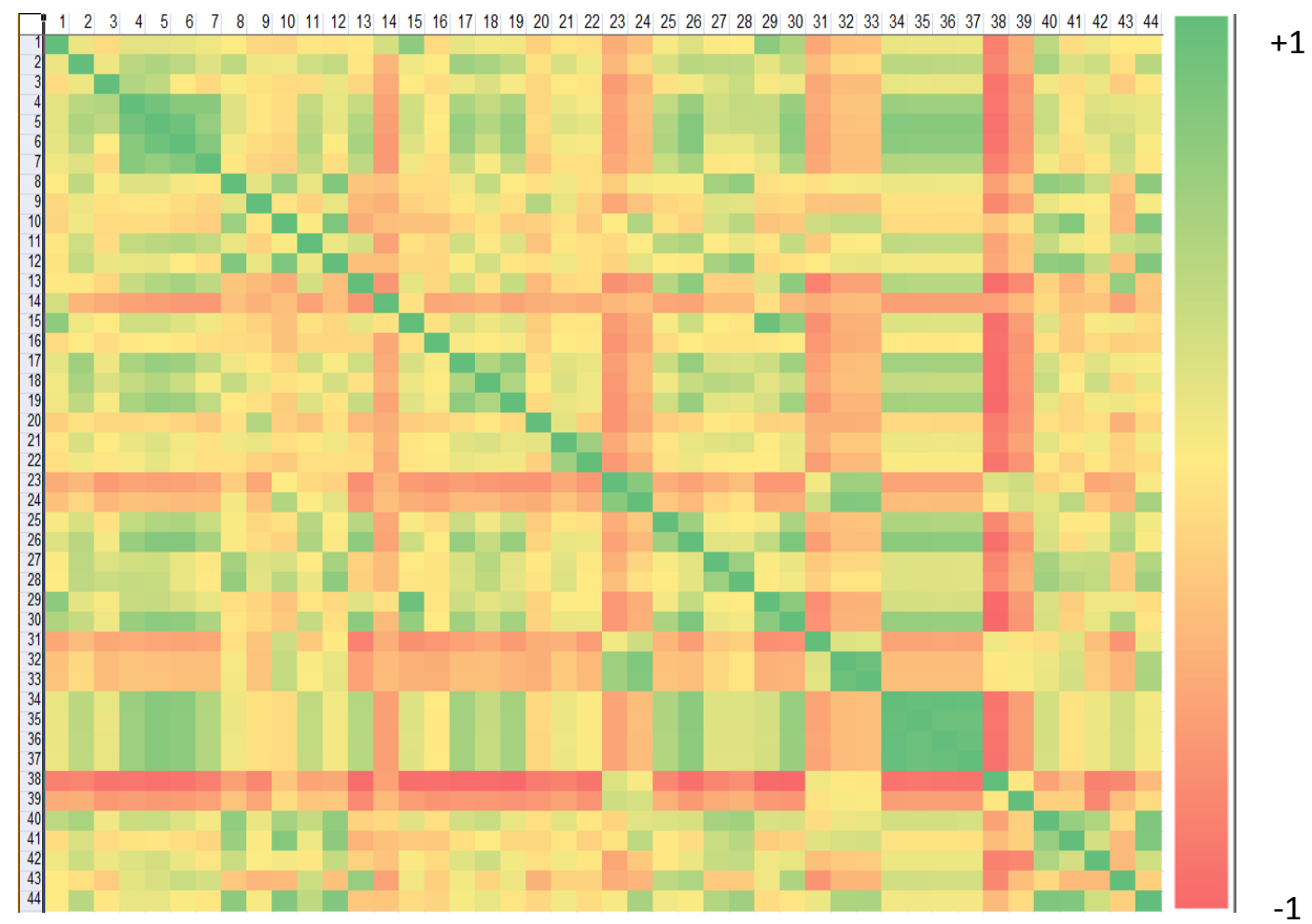

Table 8: Correlation matrix of SET-44 measurements

From the pair-wise scatter plots in Figure 2, we get an idea of relations in the chosen measurements. We observe that some measurements have linear dependence, while some are randomly distributed.

For example if we consider body height and elbow height or eye height, the plots show a linear dependence between them. This is because for tall persons, it is expected to have more eye height or elbow height. These features are highly correlated. Moreover, if we look at the head/face variation with body height, they are randomly distributed. This is due to the reality that a tall person needn't have all his measurements large, or vice versa. The head/face 
measurements are independent of the body height measurement, and there is less correlation between such features.

\subsection{Feature Extraction}

In most practical applications, the original input variables are preprocessed to transform them into some new space of variables. This reduces variability within each class and makes pattern classification much easier. This pre-processing stage is called feature extraction. Preprocessing is also used sometimes to speed up computation e.g., using only selected extracted features. Care must be taken during preprocessing because it is possible that important information is discarded. This results in decreasing the accuracy of the system. Even our work is based on 99 features obtained from CAESAR database. We need to do feature extraction as many features can be redundant. As mentioned before in Section 4.3, we normalize the complete dataset using two normalization methods as in Equation (2). We first select 11 measurements from the original dataset. From these features, we extract other features as follows:

(i) Difference from global mean for each feature - feature vector with 11 variables

$$
d_{i}^{\text {global }}=f_{i}-\mu_{i}, \quad i \in[1,11]
$$

where $f_{i}$ is one of the feature in SET-11 and $\mu_{i}$ is global mean of that particular feature

(ii) The measurements are grouped into sub-groups, say 10, based on their values. We compute the differences of sub-groups from corresponding local means - feature vector with 11 variables

$$
d_{i}^{\text {local }}=f_{i}-\mu_{k}^{\text {local }}, \quad i \in[1,11], k \in[1,10]
$$

where $f_{i}$ is one of the feature in SET-11 and $\mu_{k}^{\text {local }}$ is the local mean of $k^{\text {th }}$ group.

(iii) Given one reference feature, we compute the expected value of other features. The expected value is obtained from pair wise relations using best-fit trendline. Now we calculate the difference between actual feature and expected/predicted value of that particular feature - feature vector with 55 variables

$$
d_{i j}=f_{i}-f_{i}^{\text {exp }}(j), \quad, \quad i \in[1,11], j \in[1,2370]
$$

where $f_{i}$ is one of the feature in SET-11 and $f_{i}^{\text {exp }}(j)$ is expected value of $i^{\text {th }}$ feature and person $j$. We thus have a feature vector consisting of SET-11 original features and $77(11+11+55)$ extracted features, making a total of 88 . On this set we carry out performance measurements. 
The principal idea behind choosing the other features in such a manner is to exploit different kinds of possible variations in the selected features.

\subsection{Dimensionality Reduction}

Dimensionality reduction is the process of reducing a large number of features into reduced number of features using either feature extraction or feature selection. It refers to preprocessing on original dataset. Performing statistical analysis on high dimensional data faces many mathematical challenges. A major problem with high dimensional datasets is not all the measured features are important in understanding the underlying phenomena of interest. It also becomes expensive to construct predictive models with high accuracy for large datasets.

Larger datasets also face setbacks while fitting a curve along the data points. Practical applications of pattern recognition deal with high dimensionality spaces. The severe difficulty that can arise in spaces of many dimensions is sometimes called as curse of dimensionality. It becomes easy to project the data from higher dimension to lower dimension for visualization and study purpose. A major linear procedure for dimensionality reduction is the principal component analysis.

Principal Component Analysis - The data transformation method used in this work is Principal Component Analysis (PCA). It is a classical statistical method to transform any possibility of correlated data into a smaller number of uncorrelated data. It performs a linear mapping of data to lower dimensions such that the variance of data in low dimensions is maximized. The correlation matrix of original data is constructed and the eigenvectors of this matrix are computed. The eigenvectors which correspond to the largest eigen values which are the principal components are used to reconstruct a large fraction of variance of original data. PCA is a non-parametric method and its applicability is limited to the assumption that the observed dataset is linear combination of certain basis. In this work we have considered principal components which account to a variance for $99 \%$ of the variance. 


\section{Chapter 5: Performance Measurement}

Performance is the criteria based on which we know whether a system is reaching its desired results or not. We want to see how good the provided metrology-based features are in distinguishing humans. First, let's discuss the algorithm used in this work.

\subsection{Synthetic Variations of Metrology}

Here we discuss the variations we injected for the performance measurement in order to obtain evaluation metrics similar to other biometric systems. The dataset we have is measurement of different body parts for each person. These are attributes of genuine persons. In the real world, there will be imposters trying to match identities with original persons. We want to capture this and thus introduced variations in each measurement of a person.

Consider a Person $A$ with measurements:

$$
M^{\mathrm{A}}=\left\{m_{1}^{\mathrm{A}}, m_{2}^{\mathrm{A}}, \ldots \ldots \ldots . m_{f}^{\mathrm{A}}\right\}
$$

where $f$ is number of features considered, $f \in[1,88]$

The above vector is measurement (can be original or extracted features) of Person A with zero variations given by $P_{0}^{\mathrm{A}}$. Let the measurements be varied by a small value $\delta$. Then the variation of Person $A$ will be given as

$$
P_{1}^{\mathrm{A}}=\left\{m_{1}^{\mathrm{A}} \pm \delta_{1}^{\mathrm{A}}, m_{2}^{\mathrm{A}} \pm \delta_{2}^{\mathrm{A}}, \ldots \ldots \ldots m_{f}^{\mathrm{A}} \pm \delta_{f}^{\mathrm{A}}\right\}, \quad 0 \leq\left(\frac{\delta_{i}^{\mathrm{A}}}{m_{i}^{\mathrm{A}}}\right) \leq \tau, \forall i \in[1, f]
$$

$\tau$ is a threshold which controls the variations. In this work we used the range $0.05 \leq \tau \leq$ 0.20 , that is $5 \%$ to $20 \%$ variation of measurements. The actual value of the added error in this range is determined using a random number generator.

For the complete CAESAR population of $\mathrm{n}$ persons, we consider " $\mathrm{N}$ " variations of each person. Hence we have one original measurement, along with $\mathrm{N}$ variations of that original measurement for each person

$$
\begin{aligned}
& \text { i.e., } \quad \text { for Person } \mathrm{A}, P_{0}^{\mathrm{A}}, P_{1}^{\mathrm{A}}, \ldots \ldots \ldots P_{N}^{\mathrm{A}} \\
& \text { for Person } \mathrm{B}, P_{0}^{\mathrm{B}}, P_{1}^{\mathrm{B}}, \ldots \ldots \ldots P_{N}^{\mathrm{B}}
\end{aligned}
$$




$$
\text { for Person } \mathrm{n}, P_{0}^{\mathrm{n}}, P_{1}^{\mathrm{n}}, \ldots \cdots \cdots P_{N}^{\mathrm{n}}
$$

$P_{0}^{\mathrm{i}}$ represents original measurements of the $i^{\text {th }}$ person.

$\tau$ and $N$ are the critical parameters in this work. We need to be careful in choosing the values for these parameters as they have a serious impact on the performance of this dataset. For a fixed value of $\tau$, after some values of $N$, the variability of distance remains same over the complete database. That is, even if we increase the number of variations of each original person, there is a saturation point of $\mathrm{N}$ after which the variability of average distance between each person and all their respective variations in the entire dataset remains constant.

For a training set of 100 people, the result for variability of distance with $\mathrm{N}$ is shown in Figure 4 for different thresholds of 0.05 and 0.1 .

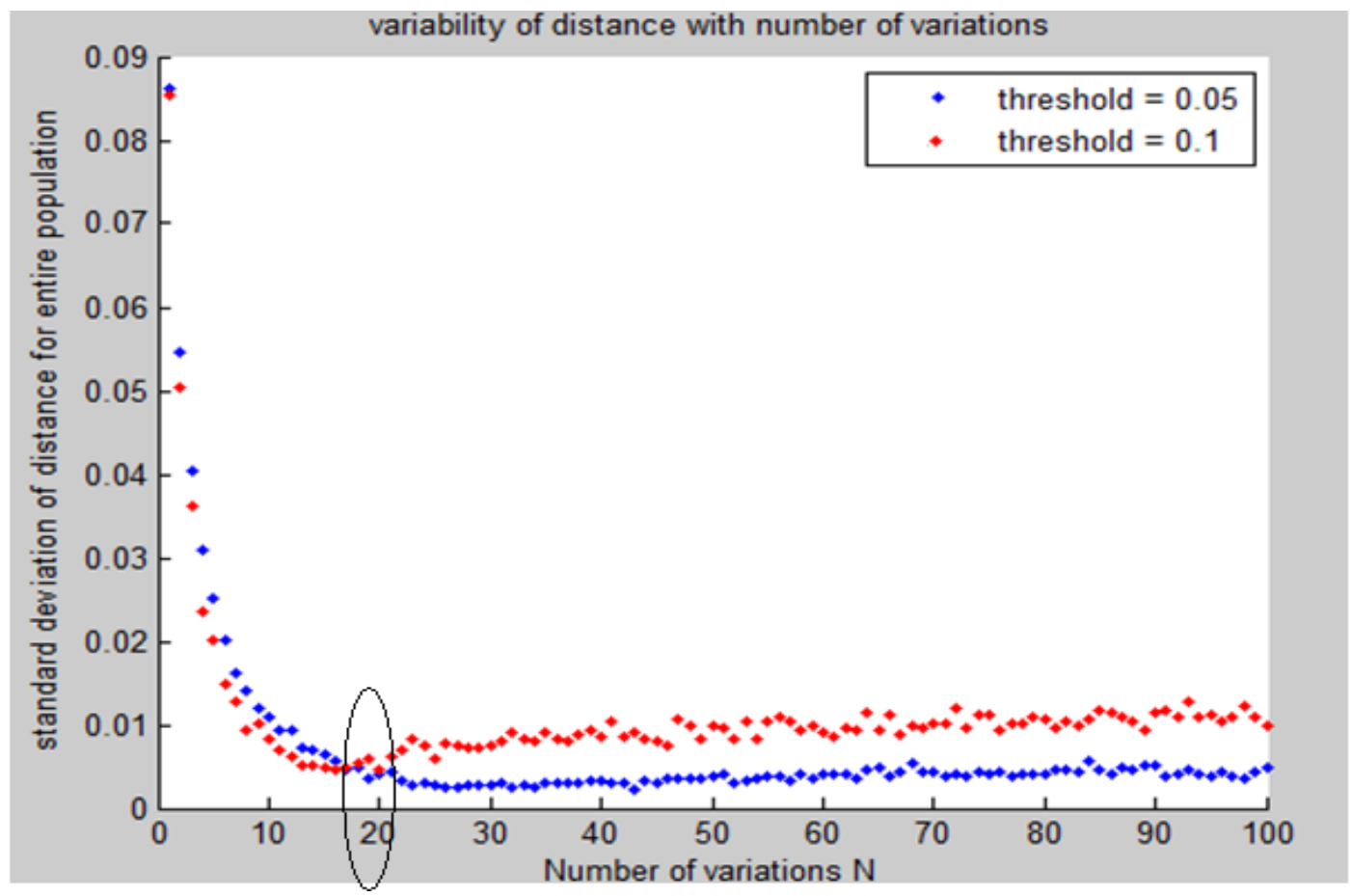

Figure 4: Variability of distance over entire population with number of variations $\mathrm{N}$

As can be observed from the above figure, if the threshold increases, the standard deviation for particular value of $N$ also increases and for both the thresholds, the deviation remains constant after $N=20$. 


\subsection{Performance Measurement Procedure}

For performance measurement of the dataset, we follow these steps:

1. Generate synthetic variations of measurements of each person.

2. We compare variations of a person with variations of other people, including original people. We store the comparisons as a distance matrix.

3. Using the distance matrix, we compute precision \& recall; genuine acceptance rate \& false acceptance rate.

4. We compute Eigen Person, Median Person, and Average Person for both Male \& Female groups.

5. For the above mentioned Persons, we plot classification rate as a function of distance threshold.

6. We also see distribution of error rates and observe genuine persons and imposters through frequency-distance plots.

\subsection{Eigen Person, Median Person and Average Person}

We divide the dataset based on gender and compute eigen Person, median Person, and average Person. These are defined as follows:

Eigen Person - Eigen person is obtained from set of eigenvectors derived from covariance matrix. Eigen person can be generated by performing PCA on the large CAESAR dataset containing different body measurements. The Eigen person is person with measurements corresponding to eigen value of each measurement.

Median Person - Median person is a single person whose measurements are median values of measurements of all persons in dataset.

Average Person - Average person represents measurements corresponding to average values of all the measurements over the complete dataset.

Given two people, say A and B, we can then evaluate how similar they are by considering their respective distances from say the eigen person. Thus we compute 4 distances as:

$$
\begin{gathered}
d_{e}(A, B)=d\left\{d\left(A, E P_{K}\right), d\left(B, E P_{K}\right)\right\}, \\
d_{m}(A, B)=d\left\{d\left(A, M P_{K}\right), d\left(B, M P_{K}\right)\right\}, \\
d_{a}(A, B)=d\left\{d\left(A, A P_{K}\right), d\left(B, A P_{K}\right)\right\}
\end{gathered}
$$




$$
\text { and } d(A, B)=d\left(P^{A}, P^{B}\right)
$$

where $d()$ represents euclidean distance between the specified persons; $E P_{K}, M P_{K}$, and $A P_{K}$ are eigen person, median person, and average person respectively, and $K=M$ or $F$ to represent Male or Female gender, respectively. $d(A, B)$ is the euclidean distance between $P^{A}, P^{B}$.

Here all the 4 metric properties are satisfied for each of the above distances. They are: (i) $d(A, B) \geq 0$ (non-negativity), (ii) $d(A, B)=0$ if and only if $A=B$ (identity of indiscernibles), (iii) $d(A, B)=d(B, A)$ (symmetry), and (iv) $d(A, C) \leq d(A, B)+d(B, C)$ (triangle inequality). Using the eigen Person, median Person, \& average Person, and the features, we can then measure the performance of metrology-based features in human classification and identification.

\subsection{Performance Measures}

Here we define the performance measures of human metrology which facilitate person identification:

\subsubsection{Distance Plots}

For the SET-11 measurements and their extracted features as discussed in Section 4.5, we compute euclidean distance over the complete CAESAR dataset. For a given person, the value in the distance plot shows the average distance between this individual and all other people. Since each individual is supposed to be distinct for every other person, if metrology features are effective in people discrimination, then each person should have a high average distance from every other person. Thus, higher values in the plot should indicate better performance. Similarly we get the distance plot for SET-44 measurements listed in Table $4 \mathrm{~b}$ over the complete dataset.

We also compute distance plots for each of the following cases to exploit different possible occurrences:

\section{Case 1: Original person against only their variaitons}

Case 2: Original person against remaining original people and their variations

Case 3: Original person against their variaitons and remaining original people and their variaitons 


\subsubsection{Classification Rate}

For the 88 features representing 11 original and 77 extracted features, we compute distances of eigen person, median person and average person based on Equation (3). Their classification rates are computed for eigen, median and average persons, the combined person and considering euclidean distances at different thresholds and plotted. Similarly, we repeat the process for SET-44 measurements.

We define the classification condition between two persons $\mathrm{A}$ and $\mathrm{B}$ as: If $d(A, B)>\tau,(\tau$ is some threshold), A and B are not similar. We call this correct classification at threshold $\tau$.

Thus the classification rate becomes

$$
\Gamma=\frac{\# \text { pairs with } d(A, B)>\tau}{\text { Total possible pairs }}=\frac{\# \text { pairs with } d(A, B)>\tau}{\left(\frac{n *(n-1)}{2}\right)}
$$

where $n$ is number of persons considered.

We later compute the combined distance of eigen Person, median Person, and average Person and get the combined classification rate of all these persons using the following equation:

$$
d(A, B)=\left(w_{e} * d_{e}(A, B)+w_{m} * d_{m}(A, B)+w_{a} * d_{a}(A, B)\right)
$$

where $w_{e}, w_{m}$, and $w_{a}$ are weights such that $w_{e}+w_{m}+w_{a}=1$; and $d_{e}(A, B), d_{m}(A, B)$, and $d_{a}(A, B)$ are obtained from Equation (3). Equation (6) is also used to perform classification.

We initially chose some random weights such that their sum is 1 . Based on few trial and error plots for the classification rate, we observed that the eigen person's classification rate is better compared to median person or average person, and median and average person's classification rates are somewhat similar. This directed to choose the weights $w_{e}=0.4, w_{m}=$ 0.3 , and $w_{a}=0.3$.

We also compute classification rate by performing optimal linear discriminant analysis (LDA) on the training set and complete set and remove any redundant features. We used the Fisher's linear discriminant analysis on the data. In this analysis, we maximize the betweenclass variance, and minimize the within-class variance. We also choose the best features from original SET-11 and SET-44 measurements using PCA, and calculate the classification rate for these PCA features. LDA has a potential advantage over PCA because PCA only finds direction of largest variance and it is an unsupervised technique. PCA doesn't include label information of the data, and these drawbacks are overcome in LDA. 
We also compute the classification rate for different age groups and each gender (male or female), as shown in the results.

\subsubsection{Precision vs. Recall}

Precision and recall are widely used measures for evaluating the accuracy of a pattern recognition algorithm. Precision is the degree of mutual agreement or repeatability among a series of individual measurements, values or results. It indicates the closeness with which measurements agree with one another. A precise estimate has small bias and variance.

Precision vs. recall graph provides an immediate, visual sense of the selected category's performance. When the plotted line is in the upper-right portion of the graph, the selected system is performing well. When the plotted line is in the lower-left portion of the graph, this indicates the system's performance is poor. We define precision and recall as follows:

$$
\begin{array}{r}
\mathrm{P}_{r}=\left(\frac{\# \text { correct retrieval }}{\text { Total retrieved }}\right)=\frac{|C|}{|T|}, \\
\text { and } \mathrm{R}_{c}=\left(\frac{\# \text { correct retrieval }}{\text { Total correct }}\right)=\frac{|C|}{N+1}
\end{array}
$$

Based on Equation (7), we compute precision and recall for 88 features and 44 measurements and plot them against each other. For the 44 features, we introduce $N$ variations and compare originals against variations of other persons too, by setting a distance threshold of 0.1 .

\subsubsection{Genuine Acceptance Rate (GAR) vs. False Acceptance Rate (FAR)}

False acceptance rate (FAR) or false match rate (FMR) is the probability that the system incorrectly matches a new data to a non-matching data present in its database. It gives a measure of invalid inputs incorrectly accepted.

False rejection rate (FRR) or false non-match rate (FNMR) is the probability that the system fails in detecting a match between a new data and its matching data present in the database. It gives a measure of valid inputs incorrectly rejected.

Genuine acceptance rate (GAR) is the overall accuracy measurement of a biometric system. We have GAR = 1 - FRR. GAR and FAR can be calculated at a particular rank or threshold as follows:

$$
G A R=\left(\frac{\# \text { correct retrieval }}{\text { Total retrieved }}\right)=\frac{|C|}{|T|},
$$




$$
F A R=\left(\frac{\# \text { falsely accepted items }}{\text { Total retrieval attempts }}\right)=\frac{|F|}{|R|}=\frac{|T|-|C|}{(N+1) *(n)-1}
$$

Figure 5 shows a general distribution of these error rates. The Receiver Operator Characteristic (ROC) curve is defined as the plot of

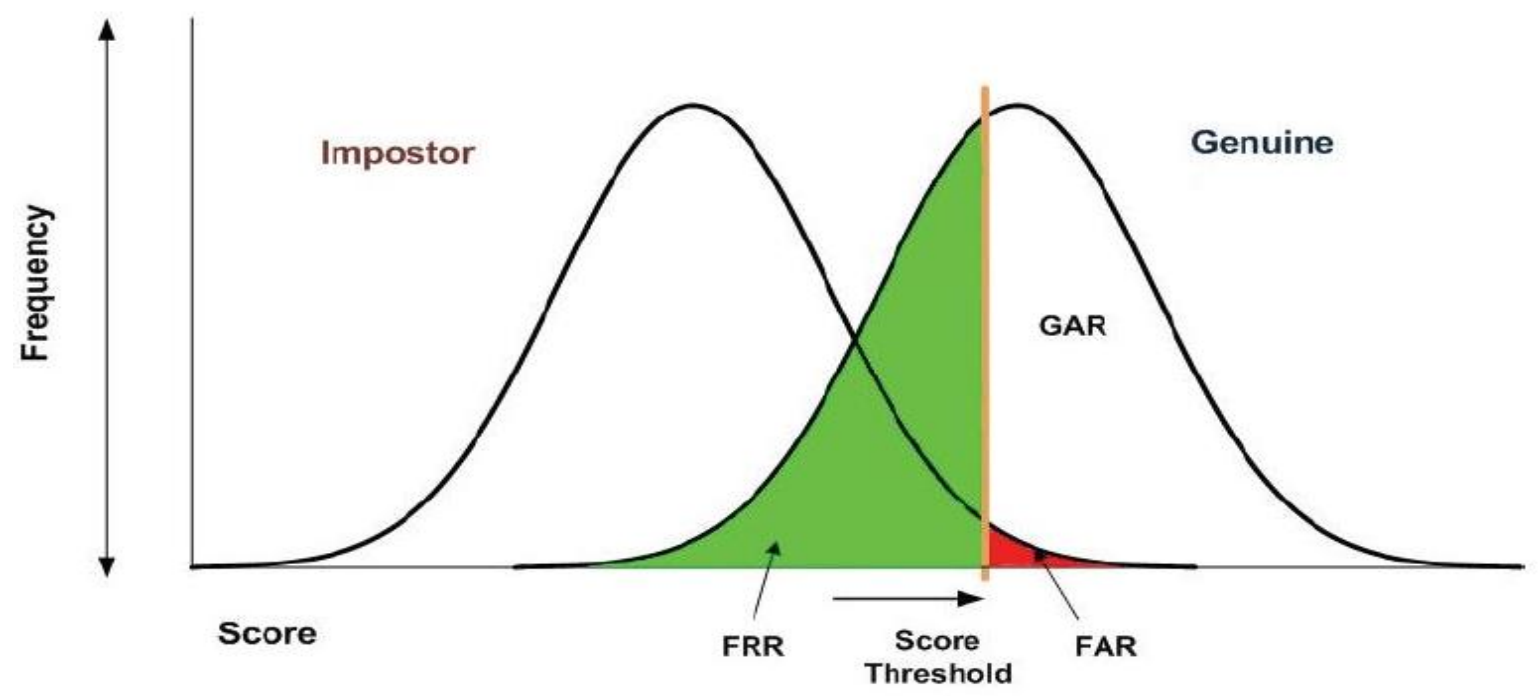

Figure 5: Relation between different error rates and their dependence on threshold

GAR against FAR. A plot between these two rates is shown in the results for both 88 features and 44 measurements.

We plot the frequency of occurrence of genuine persons and imposters. For this we consider 50 variations of each person and compare these variations with all original persons and their variations too.

For biometrics, GAR-FAR is more relevant because it helps in recognizing genuine persons and imposters. This is the key reason in designing a biometric system. Precision-recall is used in pattern recognition and machine learning to verify the correctness of an algorithm in retrieving data. The principal reason to introduce precision-recall in this work is to verify how correctly the algorithm is able to retrieve an original person's variations in a pool of many possible cases. The computational cost however depends on size of the data because more memory is needed to store large values and later test those values for correct retrieval.

\subsubsection{Clustering}

Clustering is an example of unsupervised learning method. Clustering deals with identifying groups, or clusters of data points in a multidimensional space. Data points in the same cluster are similar in some meaningful sense. Clusters consist of data points whose inter- 
point distances are small compared to the distances to points outside the cluster. In this work we use $K$-means clustering and agglomerative clustering. We did clustering on 88 and 44 measurements as shown in the results.

We have used kmeans function in MATLAB to cluster the data. The output is a vector of indices indicating to which of the $K$ clusters a point has been achieved. The method creates a single level of clusters. $K$-means computes cluster centroids differently for each measure. It uses an iterative algorithm which minimizes the sum of distances from each point to its cluster centroid for all the clusters. The algorithm moves points between clusters until the sum cannot be decreased further. This results in a set of clusters which are as compact and well separated as possible. A notable feature of $K$-means algorithm is at each iteration, every data point is assigned to one and only one cluster.

Agglomerative is a bottom-top method where each cluster is divided into sub-clusters. An advantage of this hierarchical method is we can generate smaller clusters which may be useful for further studies. But we have to know the number of clusters before hand.

\subsection{Results}

In this section, we show the results related to the performance measurement of human metrology. We used a training set of 100 persons (50 male and 50 female). In some cases, we also used the complete dataset of 2370 people. The plots are divided into two groups based on (i) training set and (ii) complete set of 2370 persons.

\subsubsection{Distance Plots}

Using SET-11 and SET-44 measurements, we show the distances for originals and each of the following variaitons:

\section{Case 1: Original person against only their variaitons}

Case 2: Original person against remaining original people and their variations

Case 3: Original person against their variaitons and remaining original people and their variaitons

We calculate the person-to-person distance for different sets of features. The results are shown in following figures. Figure 6 shows the plots for originals. 


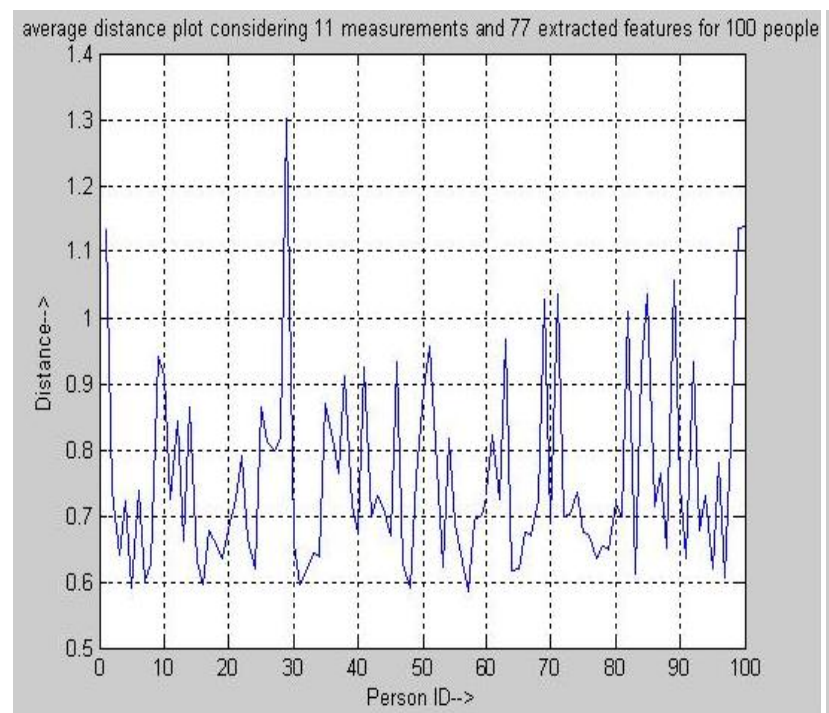

(a) 88 measurements and 100 people

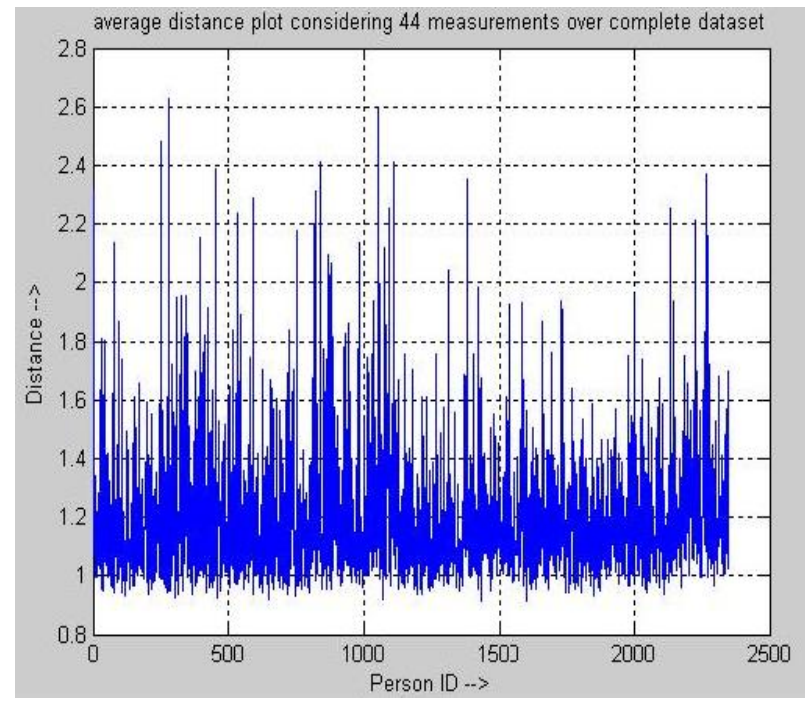

(c) 88 measurements over complete set

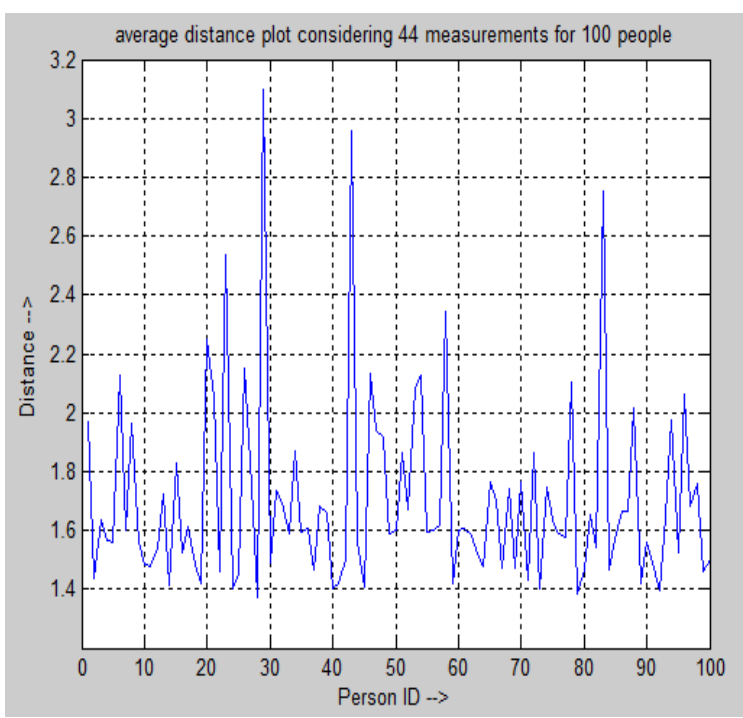

(b) 44 measurements and 100 people

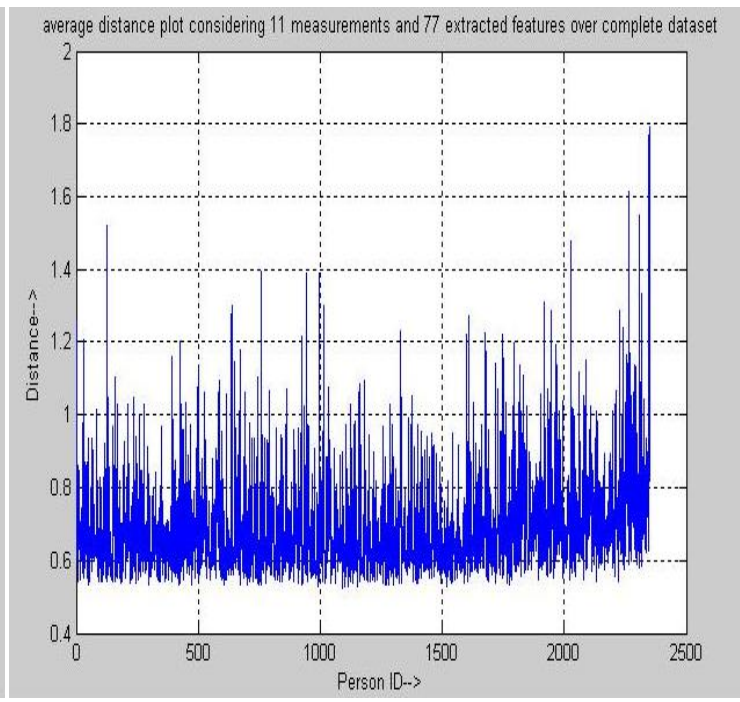

(d) 44 measurements over complete set

Figure 6: Average person-to-person distance among the population considering originals

Figure 6 shows the average person-to-person distance of original persons within the population for both training and complete set. The key observation is that the minimum distance is not zero. That is, at a certain threshold, it may be possible to separate different individuals to some extent, based on metrology features. We can also observe that the average person-to-person distance increases if the number of features considered increases. We get an idea of minimum, maximum and average distances, for each set of measurement selected, and also the number of persons. Training set contains 100 persons and complete set contains 2370 persons. The values are listed in Table 9 for both SET-11 and SET-44 measurements. 


\begin{tabular}{|c||c|c|c|c|c|c|c||}
\hline $\begin{array}{c}\mathbf{8 8} \\
\text { measurements }\end{array}$ & $\begin{array}{c}\text { Max. } \\
\text { Distance }\end{array}$ & $\begin{array}{c}\text { Min. } \\
\text { Distance }\end{array}$ & $\begin{array}{c}\text { Avg. } \\
\text { Distance }\end{array}$ & $\begin{array}{c}\mathbf{4 4} \\
\text { measurements }\end{array}$ & $\begin{array}{c}\text { Max. } \\
\text { Distance }\end{array}$ & $\begin{array}{c}\text { Min. } \\
\text { Distance }\end{array}$ & $\begin{array}{c}\text { Avg. } \\
\text { Distance }\end{array}$ \\
\cline { 1 - 3 } & 1.3020 & 0.5853 & 0.7574 & Training Set & 3.0959 & 1.3718 & 1.7028 \\
\hline Complete Set & 1.7979 & 0.5251 & 0.6945 & Complete Set & 2.6273 & 0.9116 & 1.1972 \\
\hline
\end{tabular}

Table 9: Maximum, Minimum, and Average distance values of 44 and 88 measurements considering originals

The above table gives an idea of the max, min and avg. values taking just the originals into consideration. Comparing the originals with variations of originals is more important because in practice, there will be some imposters trying to act as an original. The distance plot for comparison of originals with variation of only the originals is shown in Figure 7.

Case 1: Original person against only their variaitons.
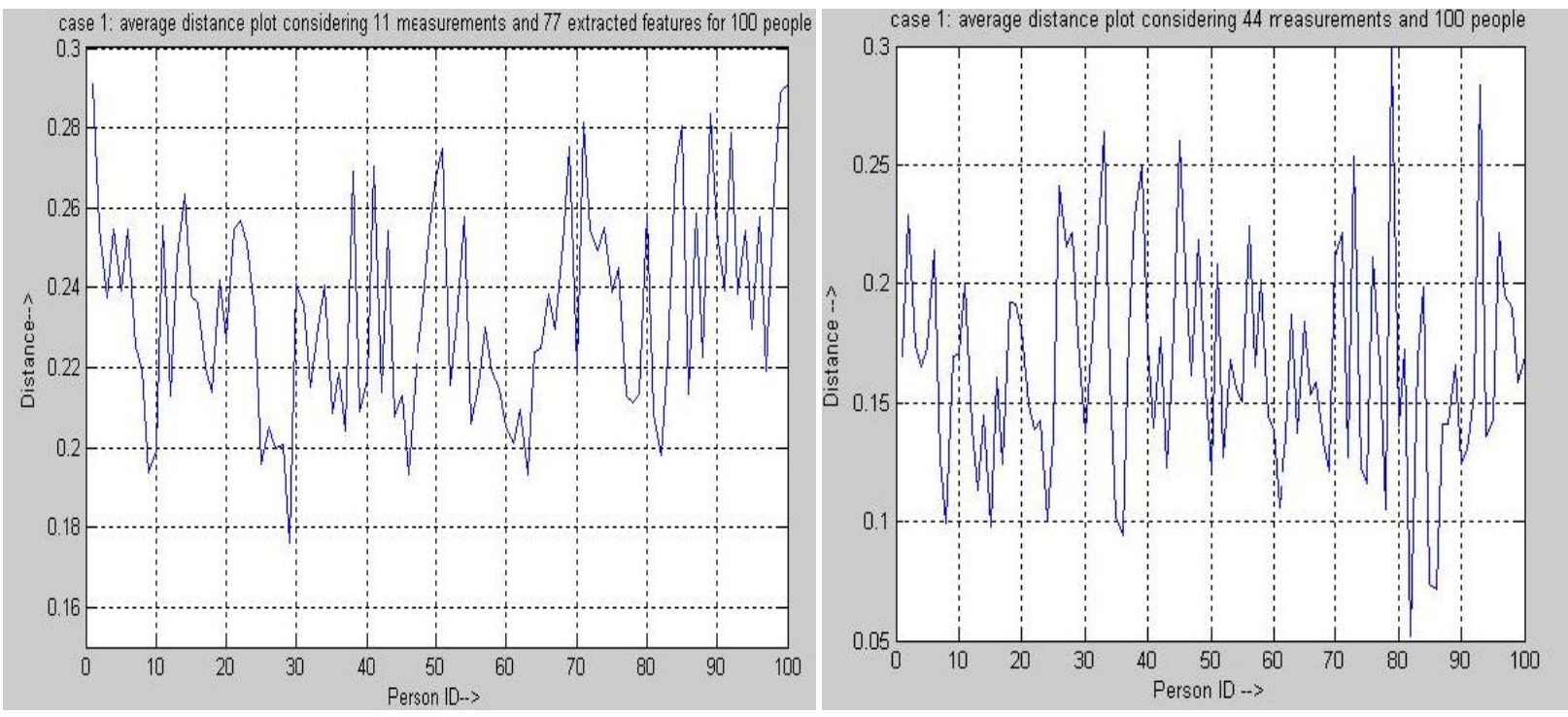

Figure 7: Average person-to-person distance for training set for Case 1

The maximum, minimum, and average value of average distances for Case 1 is shown in Table 10.

\begin{tabular}{|c||c|c|c||c||c|c|c||}
\hline $\begin{array}{c}\mathbf{8 8} \\
\text { measurements }\end{array}$ & $\begin{array}{c}\text { Max. } \\
\text { Distance }\end{array}$ & $\begin{array}{c}\text { Min. } \\
\text { Distance }\end{array}$ & $\begin{array}{c}\text { Avg. } \\
\text { Distance }\end{array}$ & $\begin{array}{c}\mathbf{4 4} \\
\text { measurements }\end{array}$ & $\begin{array}{c}\text { Max. } \\
\text { Distance }\end{array}$ & $\begin{array}{c}\text { Min. } \\
\text { Distance }\end{array}$ & $\begin{array}{c}\text { Avg. } \\
\text { Distance }\end{array}$ \\
\hline Training Set & 0.2910 & 0.1761 & 0.2344 & Training Set & 0.2976 & 0.0519 & 0.1648 \\
\hline
\end{tabular}

Table 10: Maximum, Minimum, and Average distance values of 44 and 88 measurements for Case 1

From the table, we observe that the values are very small. This is expected because the persons will be just variations of originals by $\tau=0.1$. We can say from the table that at a 
threshold of 0.1761 , all the persons (originals and their respective variations) in this case can be distinguished.

We later consider Case 2, where originals are compared with remaining originals and their variations. The distance plots are shown in Figure 8.
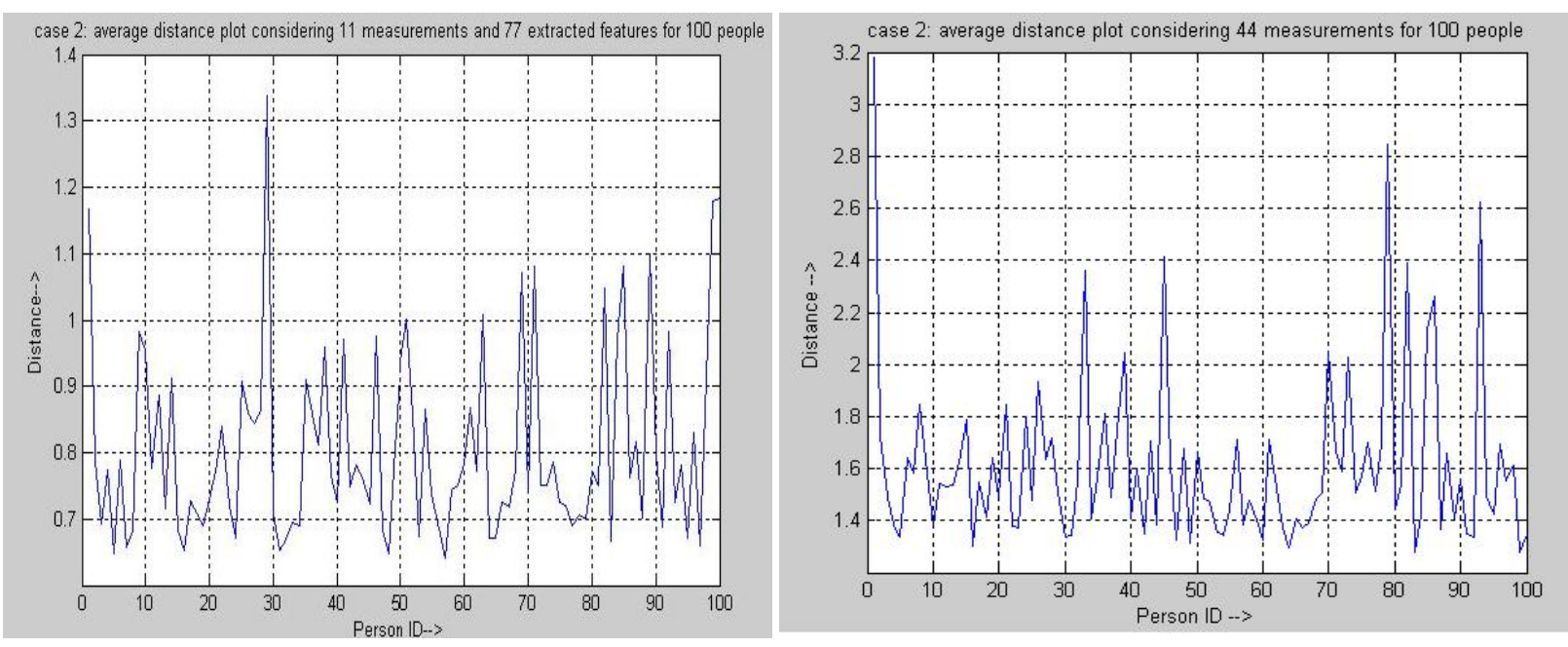

Figure 8: Average person-to-person distance for training set for Case 2

The table values are shown in Table 11.

\begin{tabular}{||c||c|c|c||c||c|c|c||}
\hline \hline $\begin{array}{c}\mathbf{8 8} \\
\text { measurements }\end{array}$ & $\begin{array}{c}\text { Max. } \\
\text { Distance }\end{array}$ & $\begin{array}{c}\text { Min. } \\
\text { Distance }\end{array}$ & $\begin{array}{c}\text { Avg. } \\
\text { Distance }\end{array}$ & $\begin{array}{c}\text { 44 } \\
\text { measurements }\end{array}$ & $\begin{array}{c}\text { Min. } \\
\text { Distance }\end{array}$ & $\begin{array}{c}\text { Avg. } \\
\text { Distance }\end{array}$ \\
Distance
\end{tabular}

Table 11: Maximum, Minimum, and Average distance values of 44 and 88 measurements for Case 2

We observe that the values increased in this case compared to Case 2. This is reasonable because here the originals are compared to originals as well as their variations.

We later consider Case 3 where originals are compared with their variations, remaining original people and their variations too. The result is shown in Figure 9. 

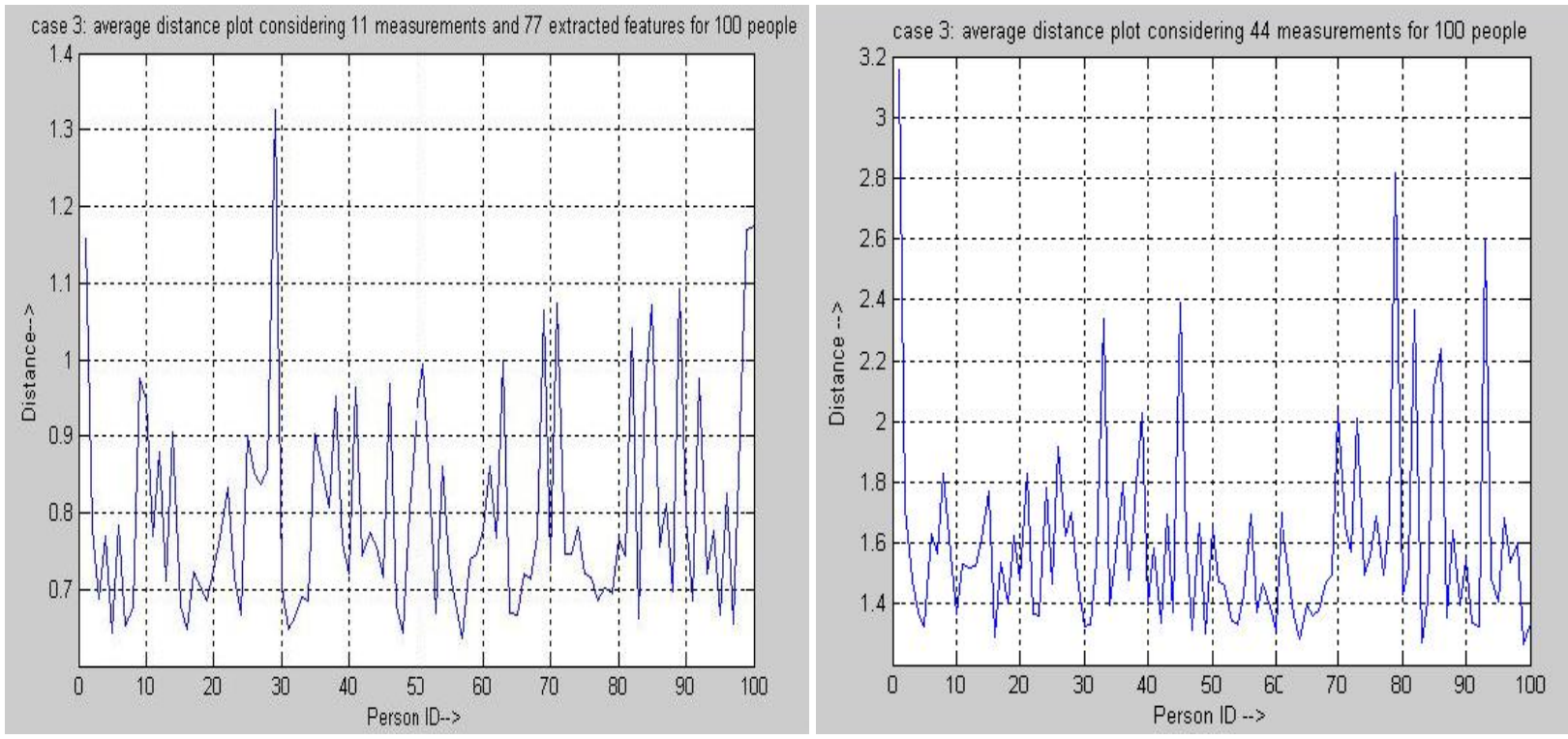

Figure 9: Average person-to-person distance for training set for Case 3

Table 12 shows the maximum, minimum, and average values of this case.

\begin{tabular}{||c||c|c|c||c||c|c|c||}
\hline $\begin{array}{c}\mathbf{8 8} \\
\text { measurements }\end{array}$ & $\begin{array}{c}\text { Max. } \\
\text { Distance }\end{array}$ & $\begin{array}{c}\text { Min. } \\
\text { Distance }\end{array}$ & $\begin{array}{c}\text { Avg. } \\
\text { Distance }\end{array}$ & $\begin{array}{c}\mathbf{4 4} \\
\text { measurements }\end{array}$ & $\begin{array}{c}\text { Max. } \\
\text { Distance }\end{array}$ & $\begin{array}{c}\text { Min. } \\
\text { Distance }\end{array}$ & $\begin{array}{c}\text { Avg. } \\
\text { Distance }\end{array}$ \\
\hline Training Set & 1.3260 & 0.6373 & 0.8008 & Training Set & 3.1538 & 1.2694 & 1.5976 \\
\hline
\end{tabular}

Table 12: Maximum, Minimum, and Average distance values of 44 and 88 measurements for Case 3

From the above table, we observe there is very small difference from values compared to Table 11. This is because of the variations of originals introduced in Case 3 .

\subsubsection{Classification Rate}

The observations on the minimal distances above imply that we could perform some classification study by varying the thresholds. Here, each person is considered a class in itself. A classification error occurs if below a given threshold more than one person is found to be similar to a given subject. The number of such errors point to the error rate. Based on Equations (4), (5) and (6), we compute the classification rates considering the same training set, and later on the complete set. We calculate it for each case 1, 2 and 3 as like the distance plots. The results are shown below.

In this figure, the classification rate is shown based on euclidean distances, eigen distances, average distances, median distances, and cosine distances and after performing LDA and PCA, as discussed in Section 5.4.2. We also make use of Equation (6) to get the combined distance. 
The results are shown in Figure 10(b) \& 10(d) for the SET-44 measurements. As can be seen from the plots, classification rate is higher for PCA and LDA until some distance threshold and falls steeply after that.

From the results we observe that euclidean distance results in the highest classification rate, and cosine distance in the lowest classification rate (Figure 10(b) \& $10(\mathrm{~d})$ ). Because of this we exclude cosine distances for the remaining classification rate plots.

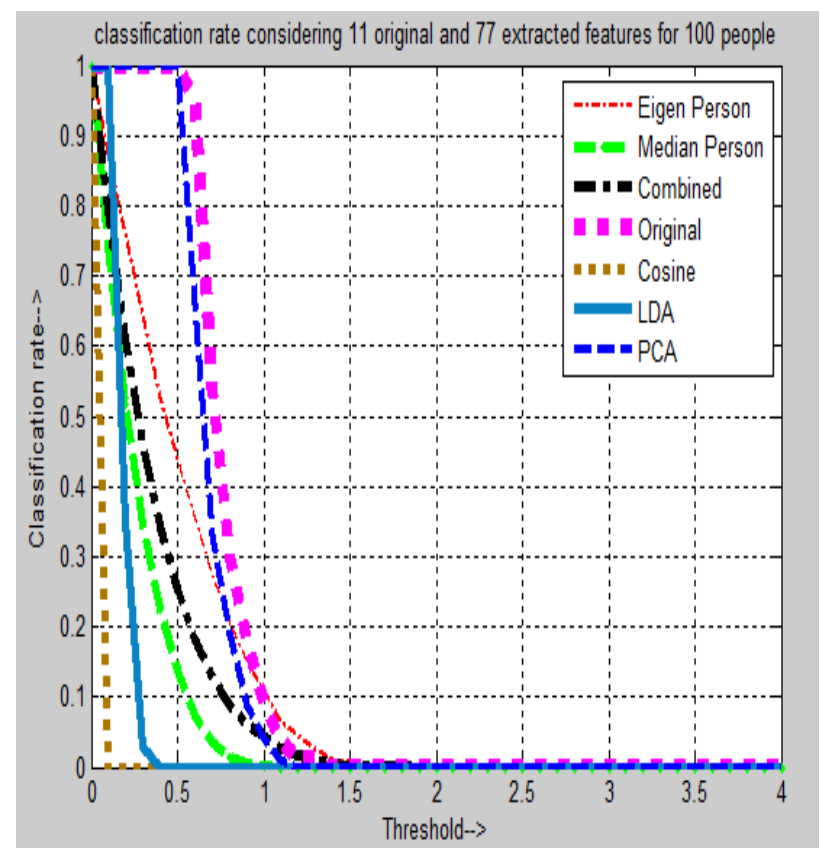

(a) 88 measurements and 100 people

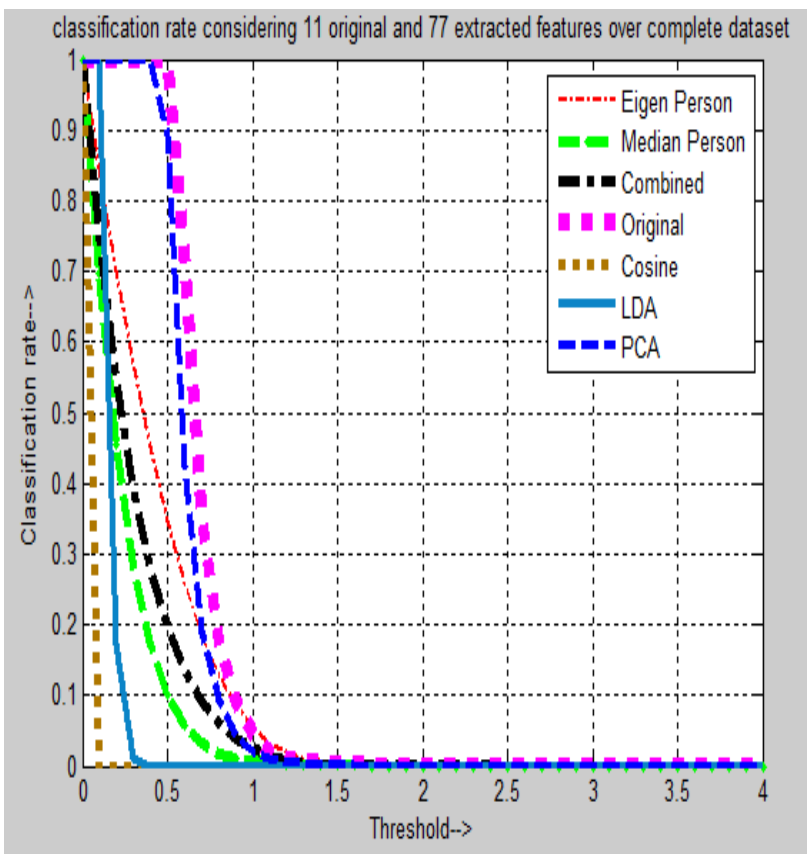

(c) 88 measurements over complete set

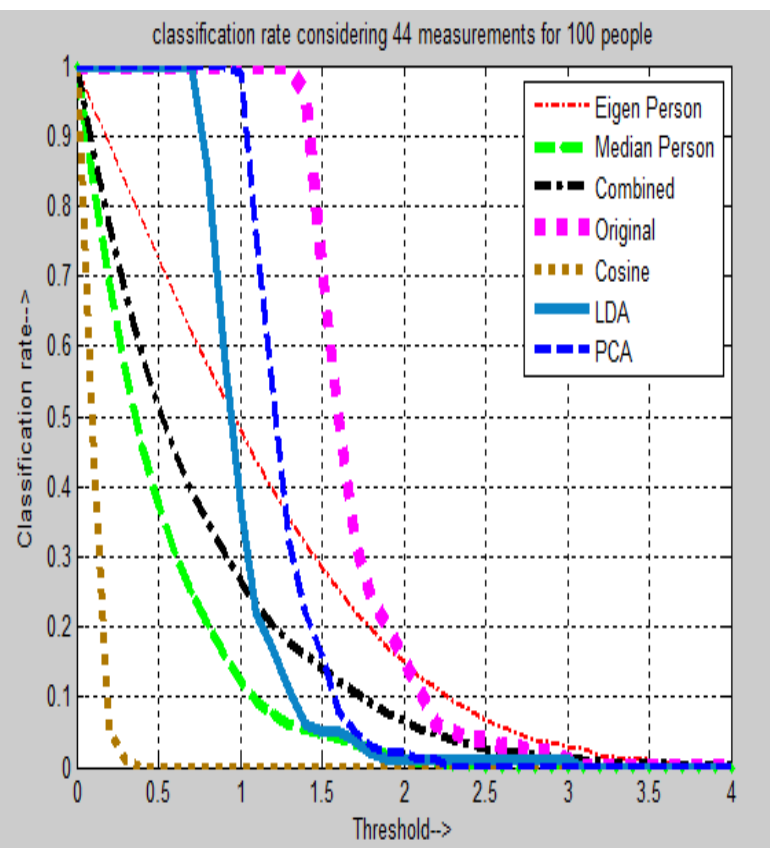

(b) 44 measurements and 100 people

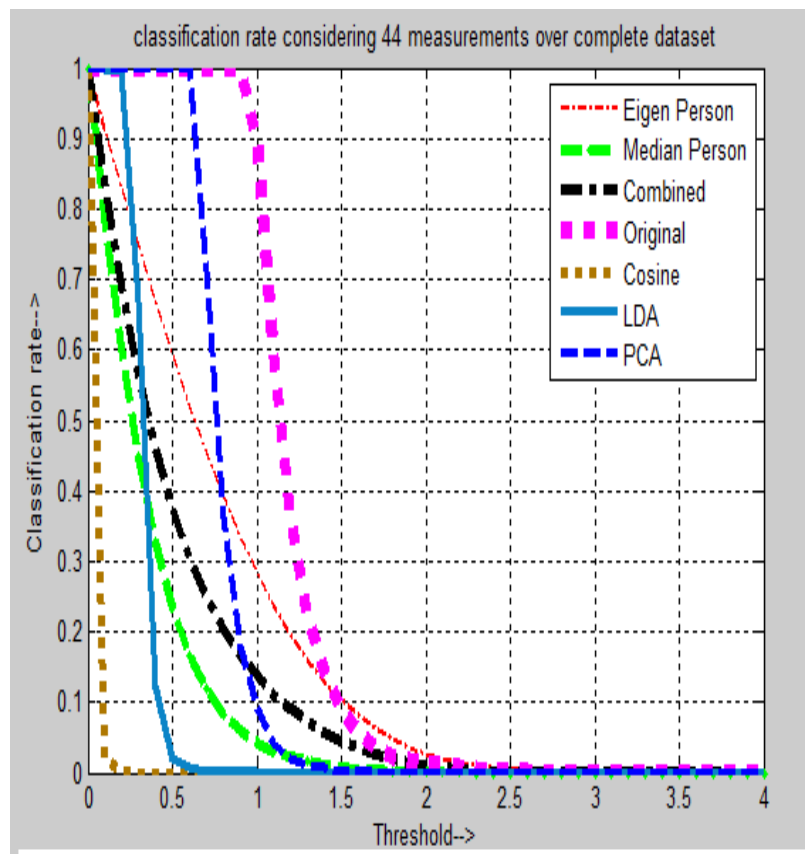

(d) 44 measurements over complete set

Figure 10: Classification rate as a function of threshold for original persons 
Figure 11 shows the classification rates for each of the cases 1, 2 and 3.

Case 1: original person against only their variations

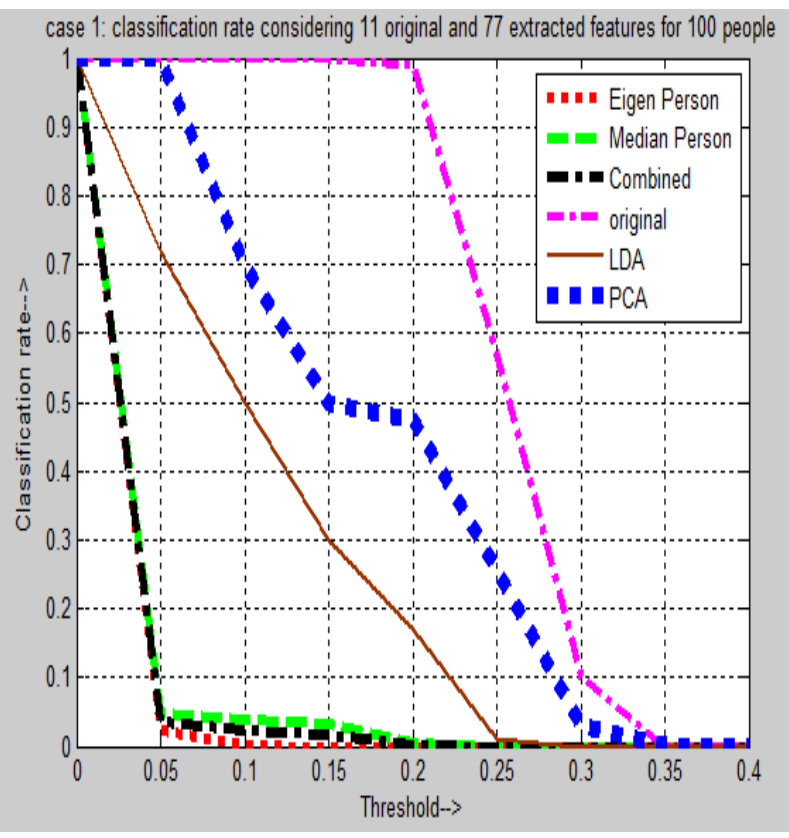

(a) 88 measurements and 100 people

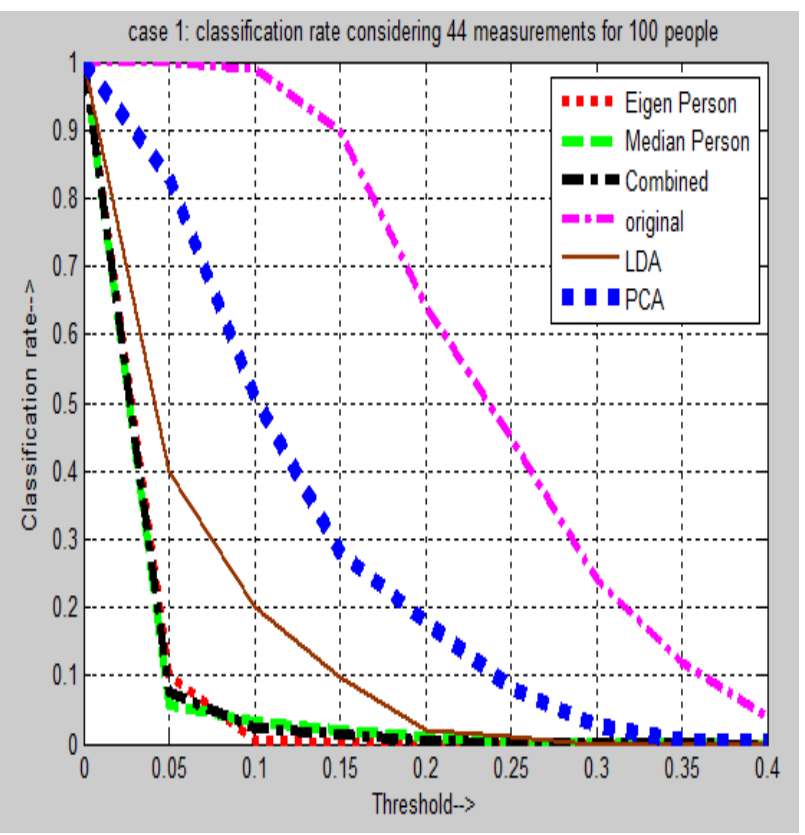

(b) 44 measurements and 100 people

Case 2: original person against all original people and their variations

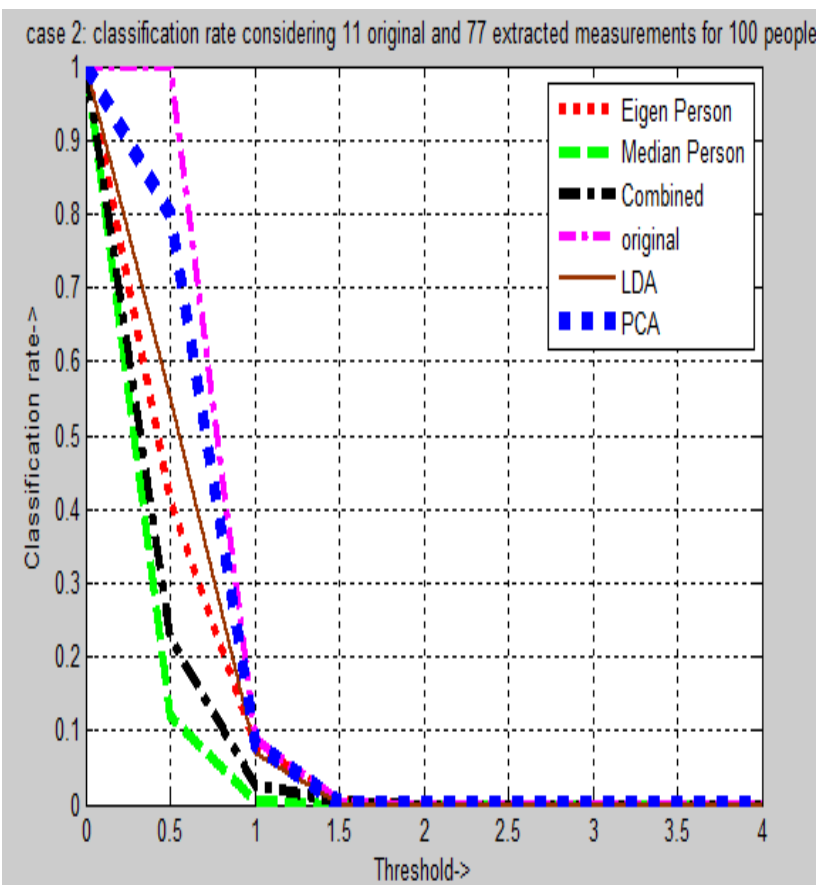

(c) 88 measurements and 100 people

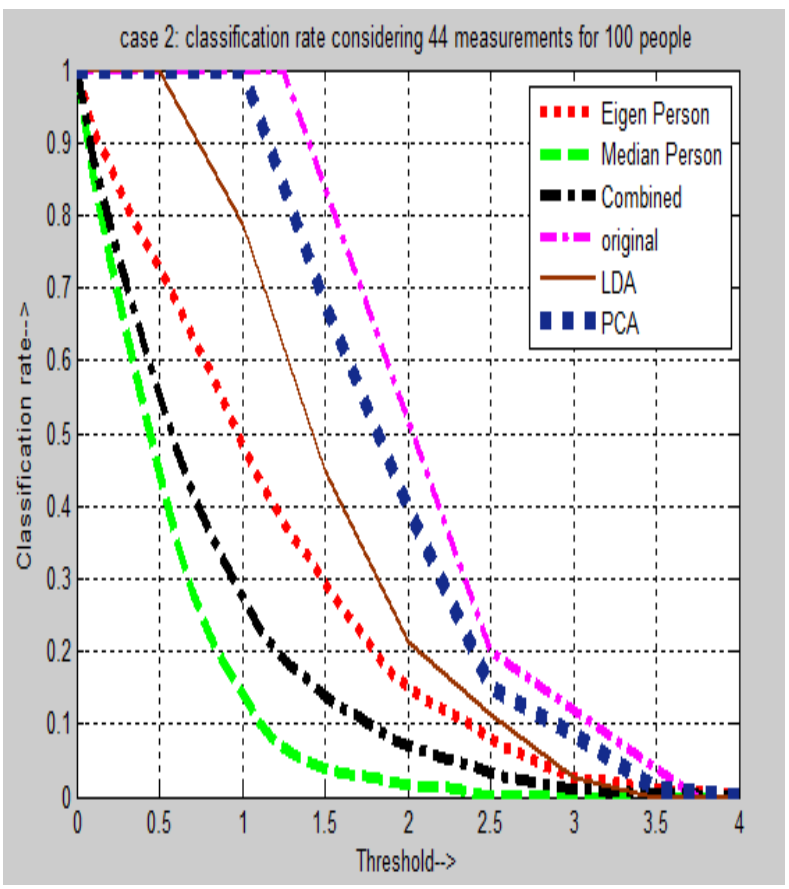

(d) 44 measurements and 100 people 
Case 3: original person against their variations and remaining original people and their variations

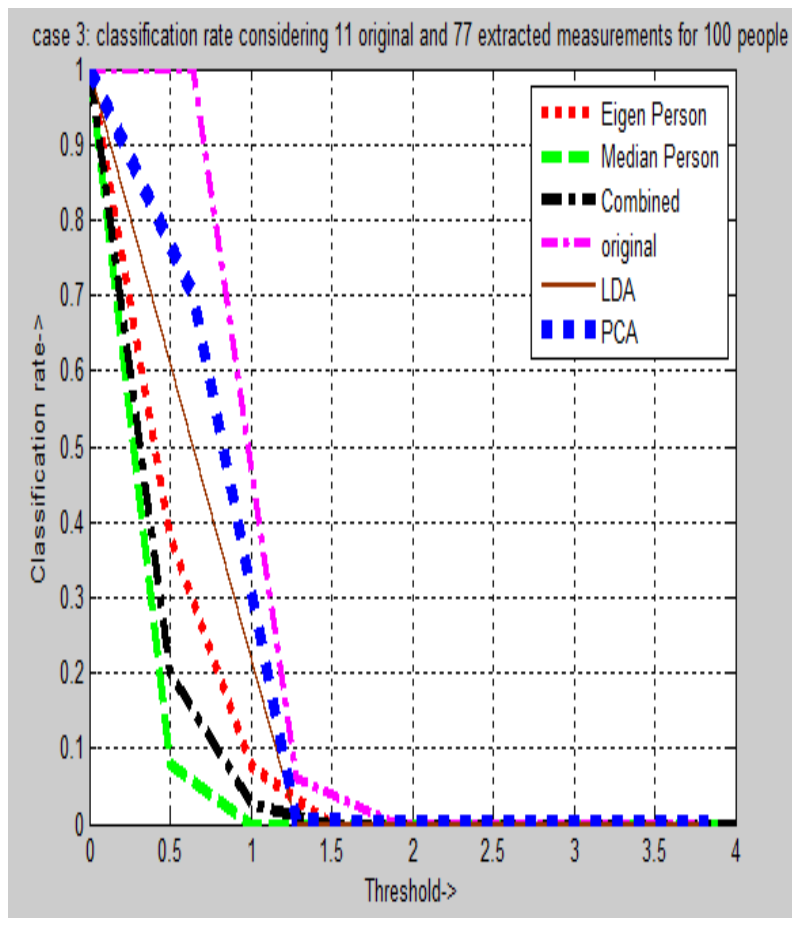

(e) 88 measurements and 100 people

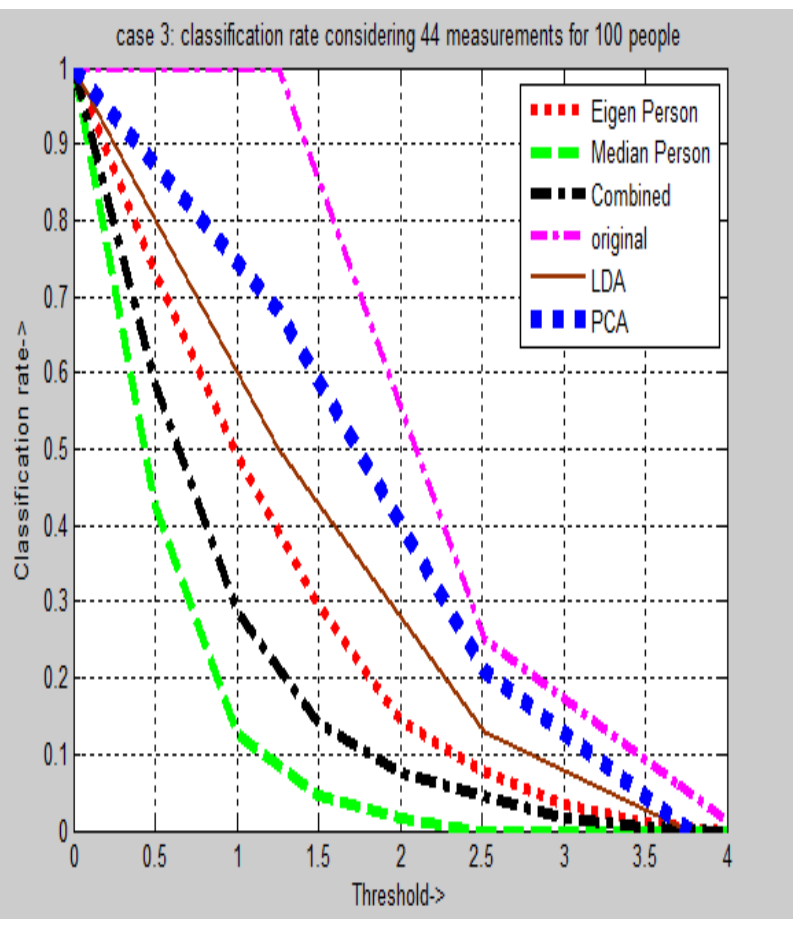

(f) 44 measurements and 100 people

Figure 11: Classification rate as a function of threshold for each case

At a particular threshold and given dataset, we can know the classification rate for different measurements in each case from the above plots. As can be observed in the above figures, the classification rate is better for SET-44 compared to 88 features (SET-11 original and 77 extracted features). Also as number of persons increase, the classification rate decreases.

\subsubsection{Factor of age and gender}

The classification plots in above figures are conditional distributions because we considered both male and female genders as a single group. We now consider each gender separately and look at their performance measure. We also consider the factor of age in distinguishing between humans. From the provided dataset, we divide the persons into following groups based on both age and gender:

(i) Age group 1 - (18 yrs to $25 \mathrm{yrs}$ ), (ii) Age group 2 - (26 yrs to $40 \mathrm{yrs}$ ),

(iii) Age group 3 - (41 yrs to 59 yrs), and (iv) Age group 4 - (above 60 yrs)

The maximum age in Male group and Female group was 79 years and 69 years respectively. We plot the classification rates for each age group ((i) - (iv)) and gender (male or female). We 
consider a training set of 50 male and 50 female in each age group. The results are shown in Figure 12. The male plots are on the left side and female plots to the right.
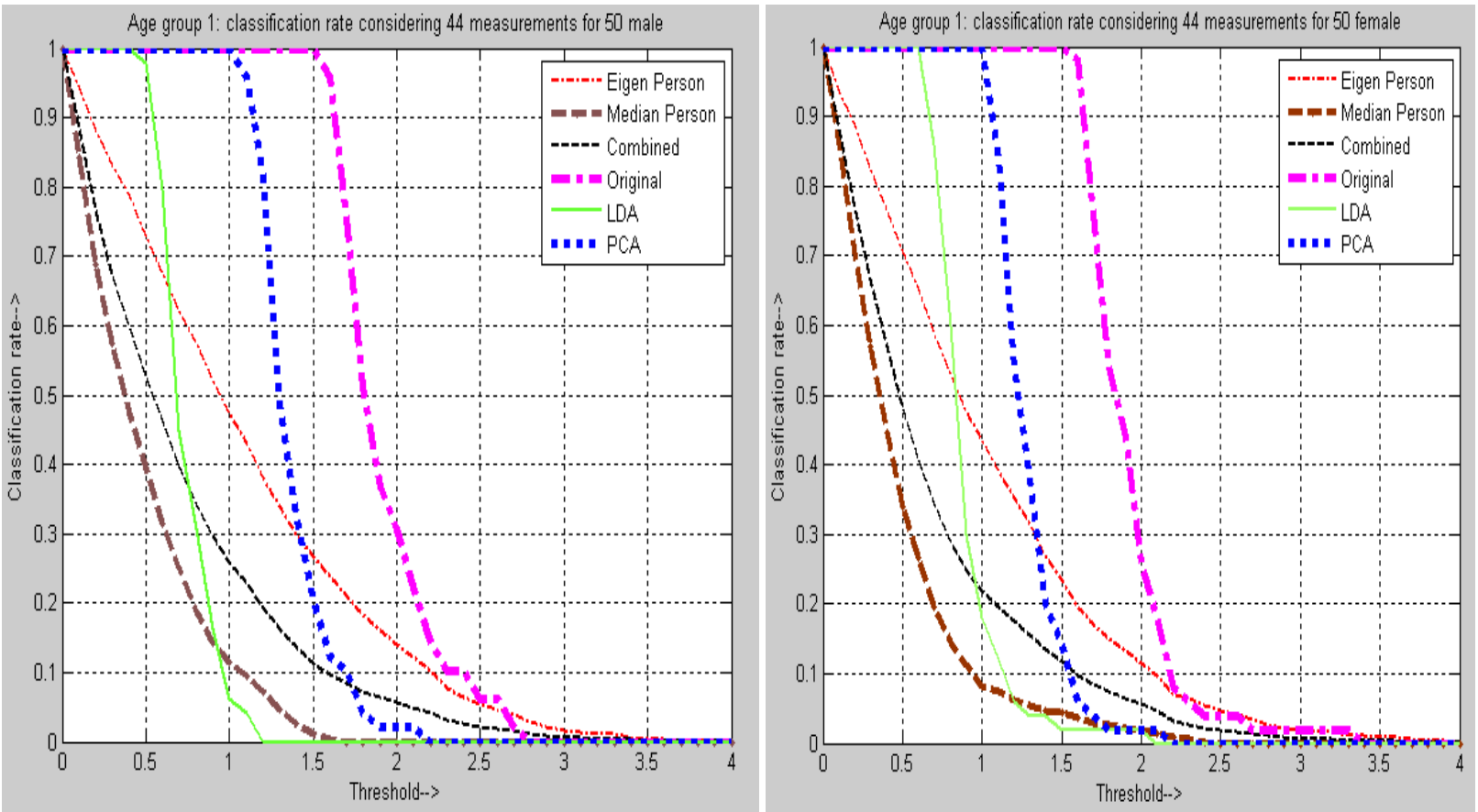

(a) Classification rate for 50 male and 50 female in age group (i) - (18 yrs to $25 \mathrm{yrs}$ )
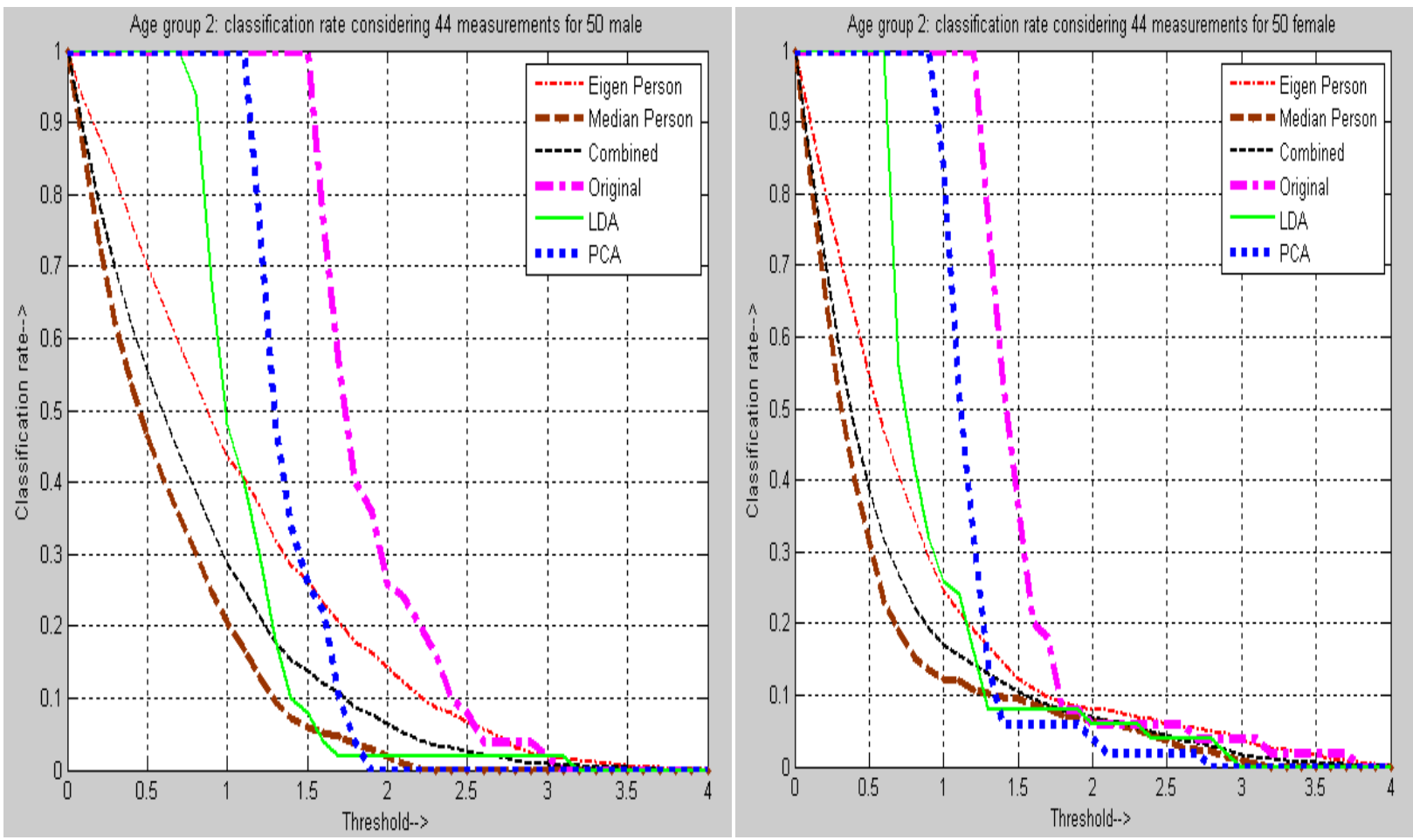

(b) Classification rate for 50 male and 50 female in age group (ii) - (26 yrs to 40 yrs) 

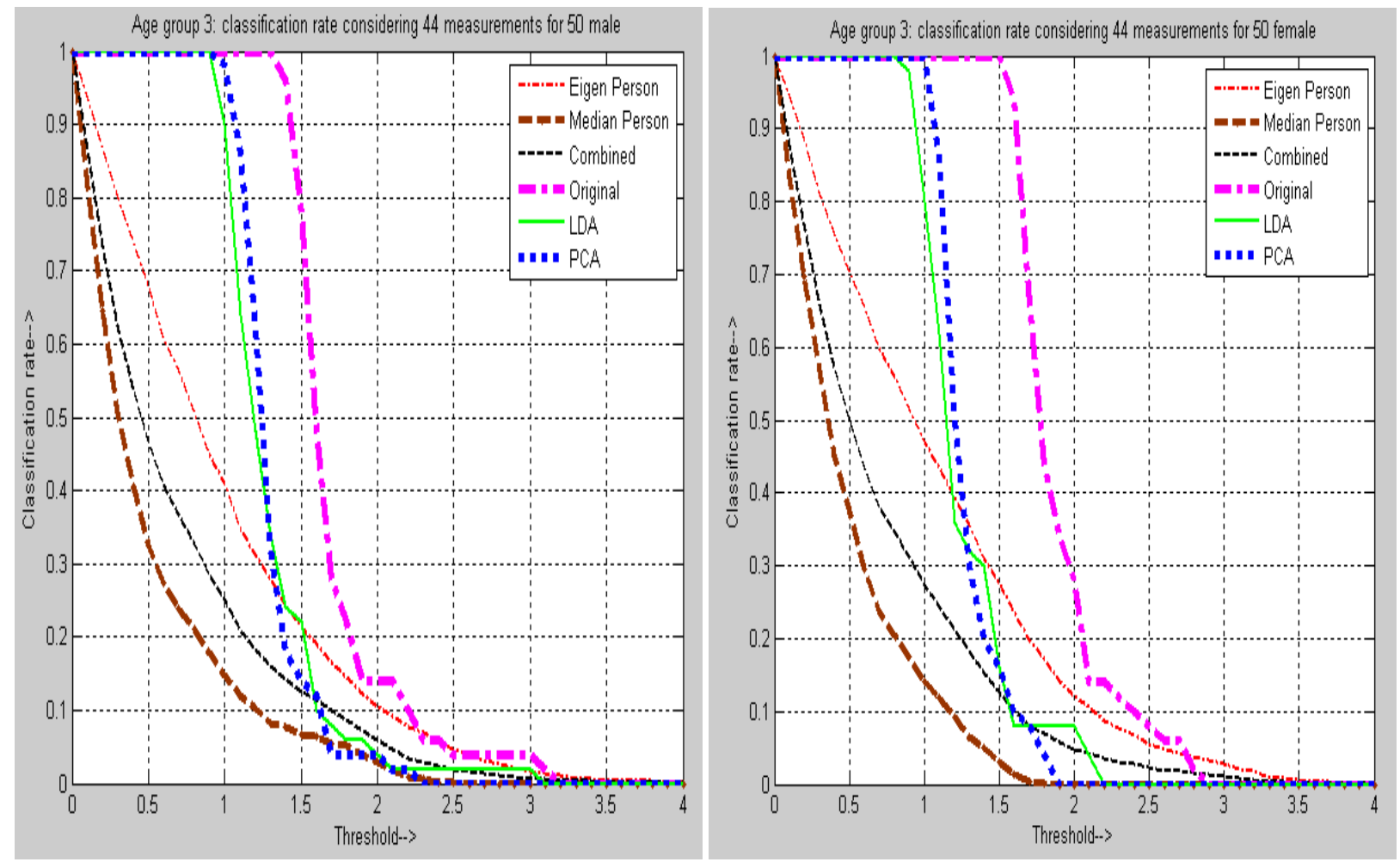

(c) Classification rate for 50 male and 50 female in age group (iii) - (41 yrs to 59 yrs)
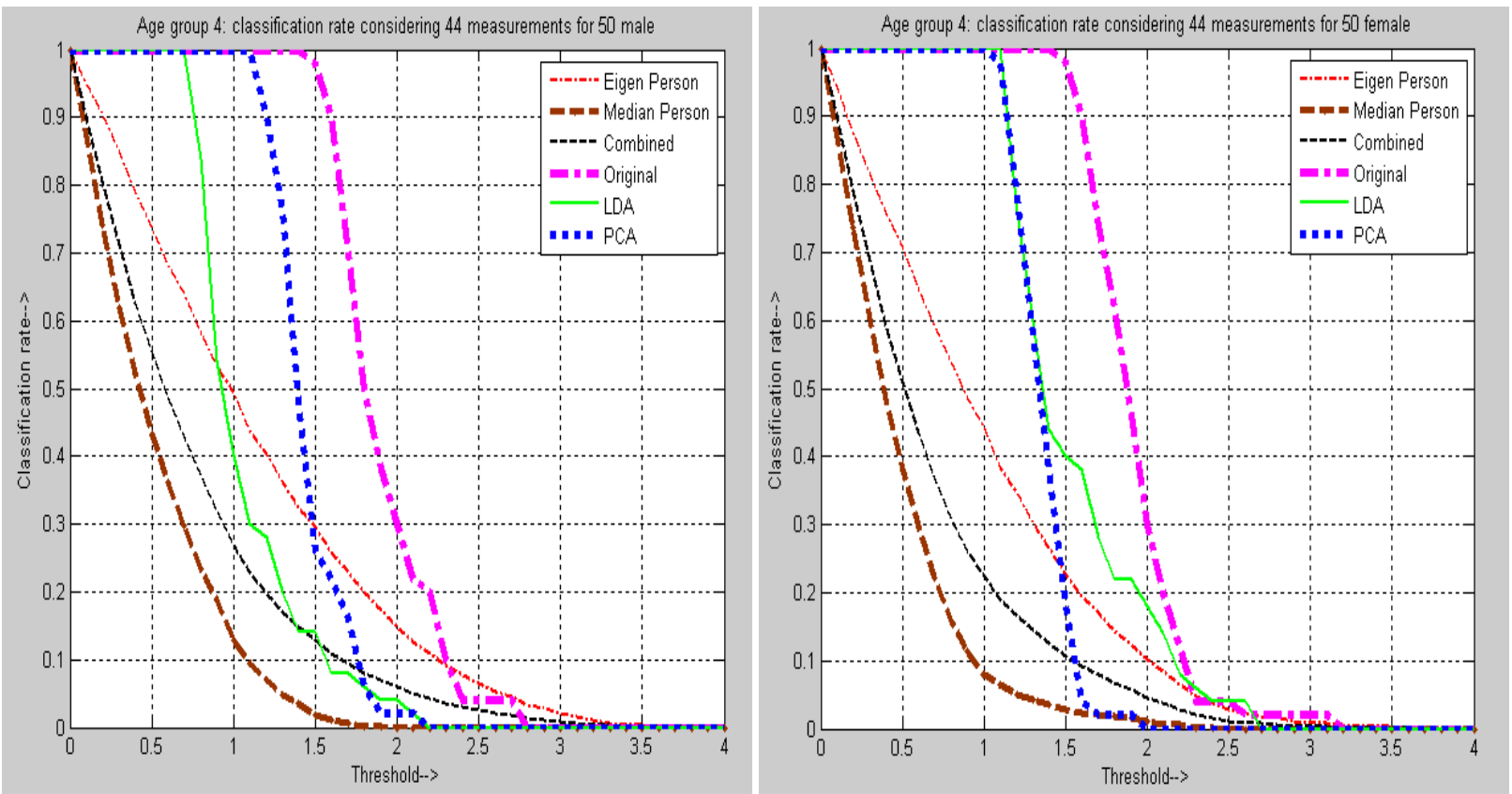

(d) Classification rate for 50 male and 50 female in age group (iv) - (above 60 yrs)

Figure 12: Classification rates for each age group considering male and female genders 
The above plots give an idea of effect of age in determining the classification rate for the chosen training set of 50 male and 50 female. A closer look at the plots suggest that for age groups of 26 to 59 years, in this training set, the classification rate for female group is higher compared to male group. For the remaining age groups, the classification rates are close for both genders.

The individual classification rate of each gender in a particular age group is less compared to classification rate of both genders combined as a single group. This is observed by comparing above figures with Figure 10, where the people were from all age groups.

\subsubsection{Precision vs. Recall}

We compute precision and recall as a characteristic performance measure. As described in Equation (7), we calculate the precision and recall assuming $N=50$ variations for each individual person, and a threshold of 0.1. The plots are shown in Figure 13. We show the plots for original and PCA features. The significance of using PCA is to discover if the selected measurements (SET-11 and SET-44) can be represented by a smaller number of measurements.

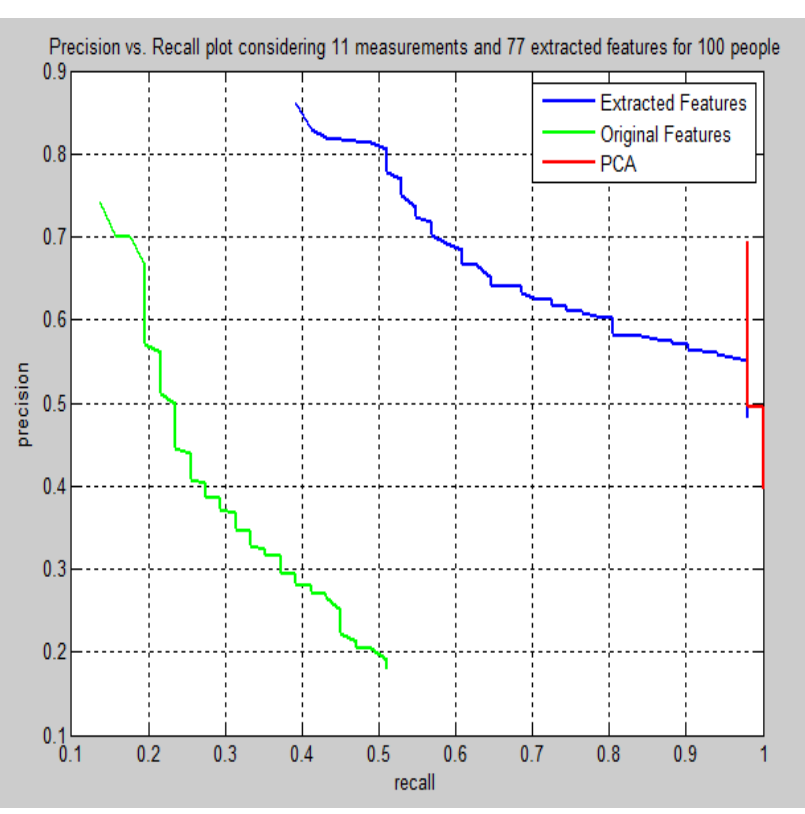

(a) 88 measurements and 100 people

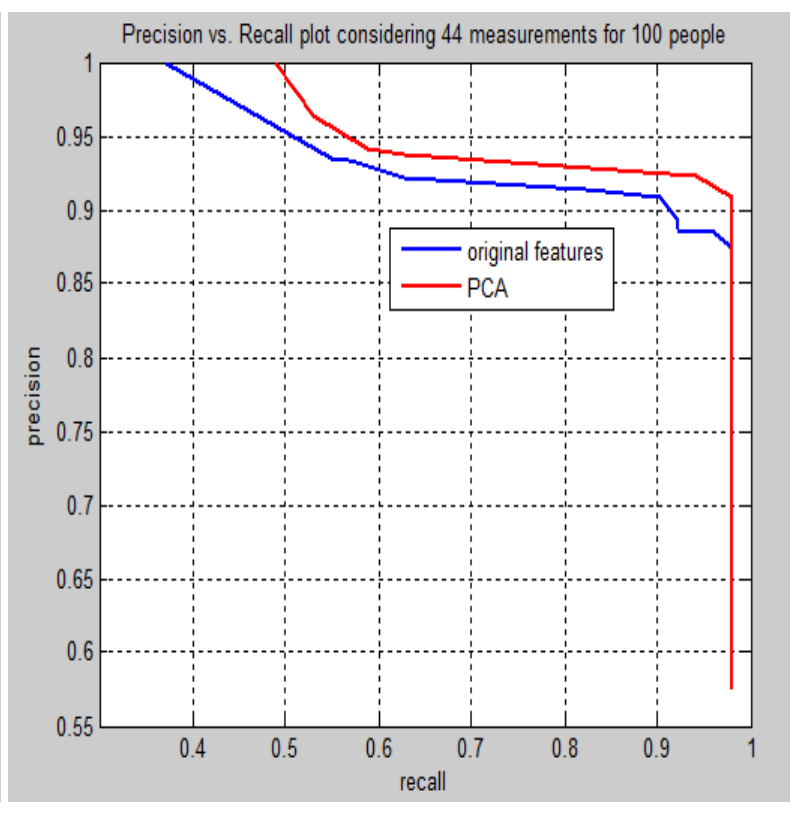

(b) 44 measurements and 100 people 


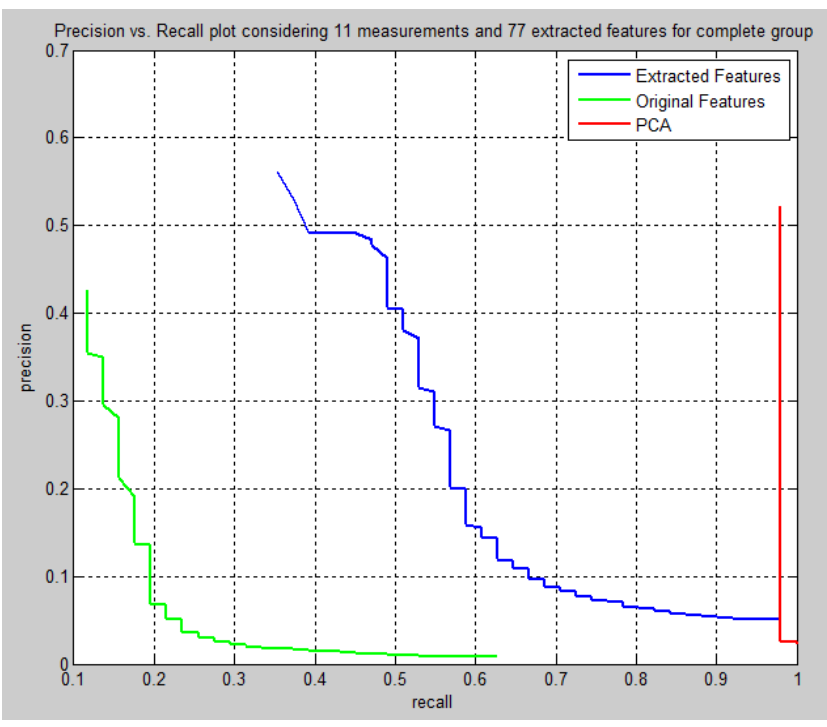

(c) 88 measurements over complete set

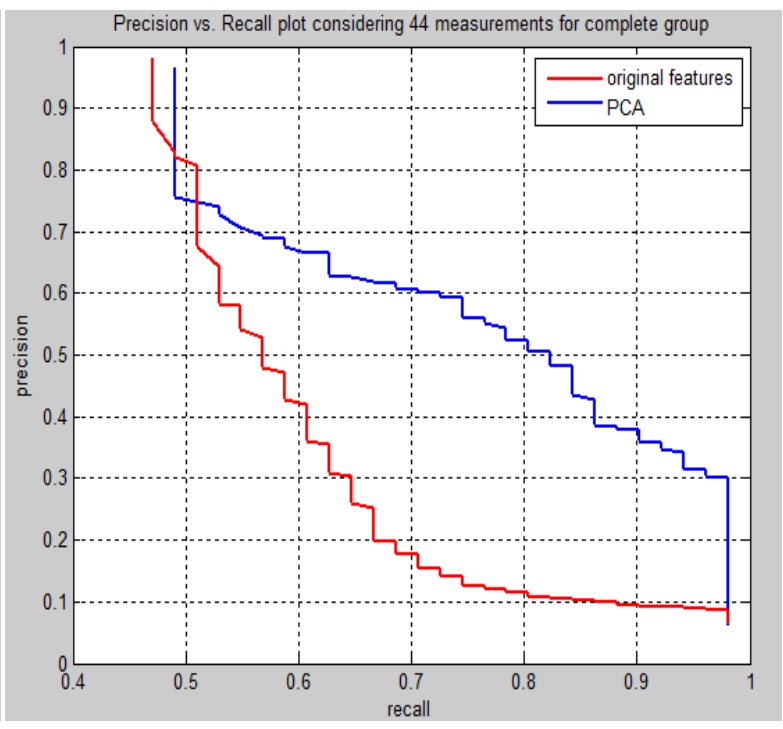

(d) 44 measurements over complete set

Fiaure 13: Precision vs. Recall curve for both trainina and comblete set

Precision-recall curve represents accuracy of a system. From above results, it can be inferred that PCA is doing much better compared to original or extracted features. Also the accuracy is more for SET-44 compared to SET-11 features.

\subsubsection{Genuine Acceptance Rate (GAR) vs. False Acceptance Rate (FAR)}

Based on Equation (8), we calculate GAR and FAR, and plot them against each other for different measurements and sets. For this $\mathrm{N}=50$ and threshold $=0.1$. The plots are shown in Figure 14.

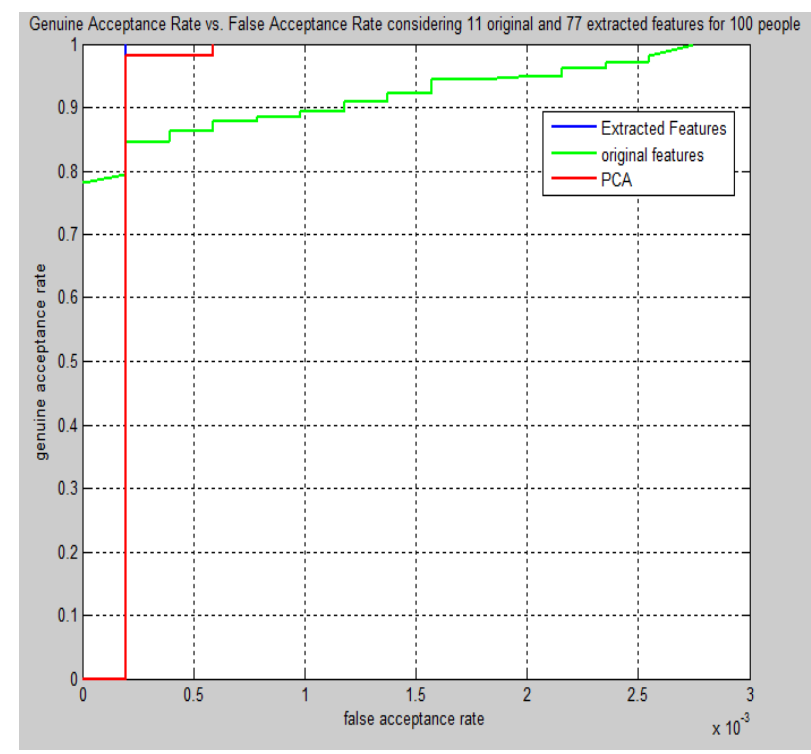

(a) 88 measurements and 100 people

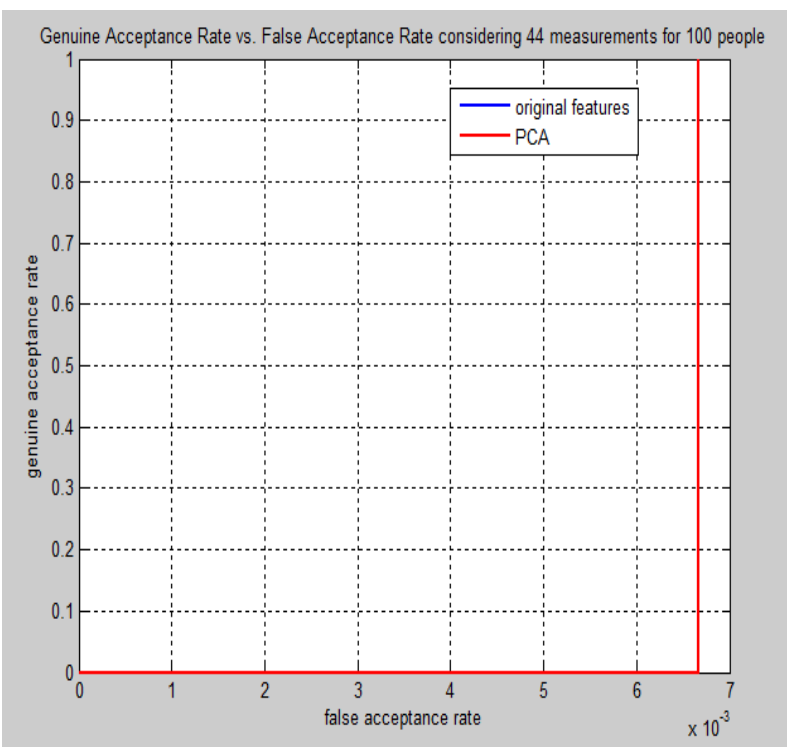

(b) 44 measurements and 100 people 


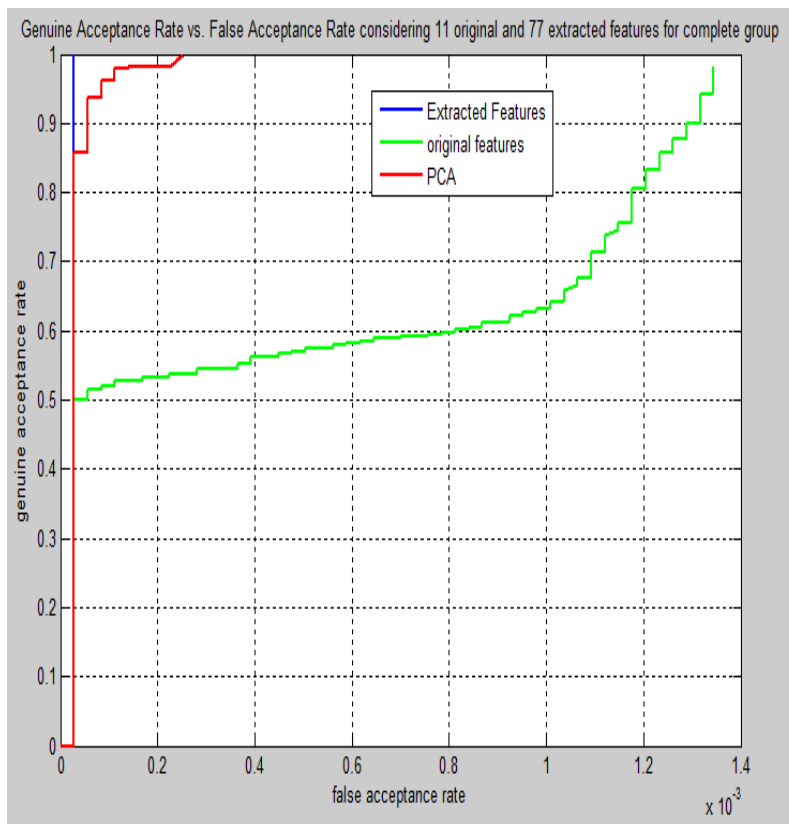

(c) 88 measurements over complete set

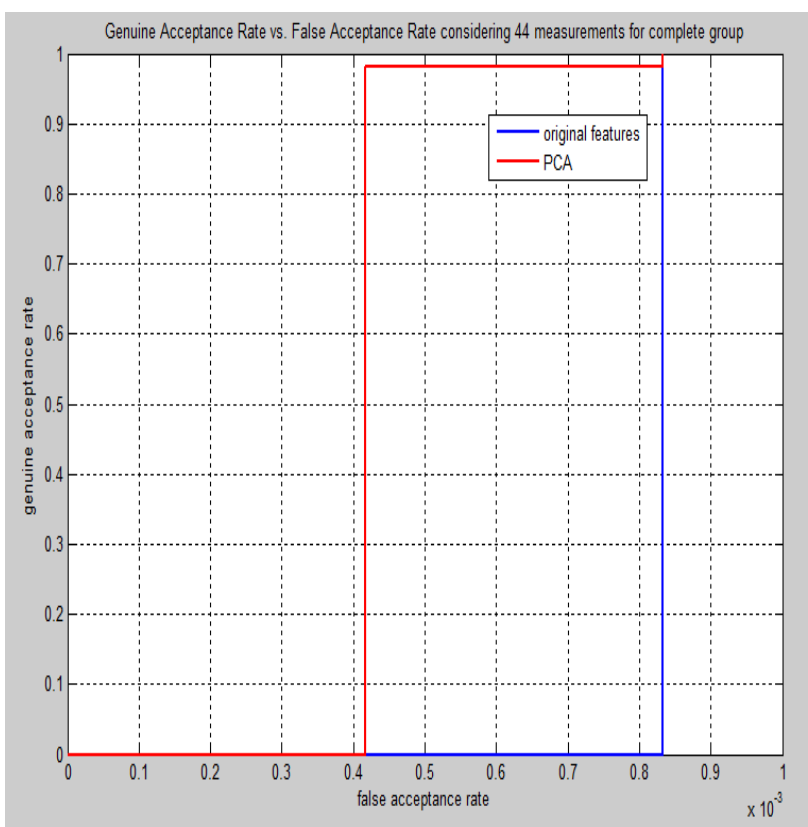

(d) 44 measurements over complete set

Figure 14: Genuine Acceptance Rate vs. False Acceptance Rate

The above results show performance based on gar and far. The performance is good when compared to other biometric systems and gives an initiative of performance of different feature sets used in the dataset.

Later we plot detailed Genuine and Imposters probabilities to observe at what distance they are occurring. We introduce 50 variations of each person, where each measurement is varied by a threshold of $5 \%$ or $20 \%$. The plots are shown in Figure 15 and Figure 16. 


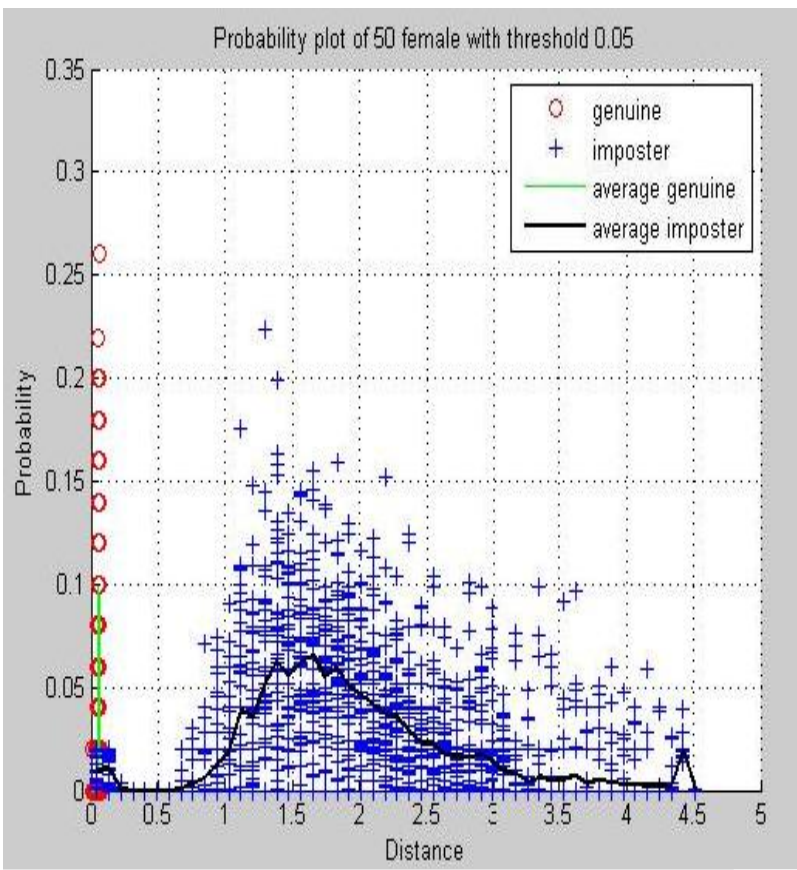

(a) 50 Female with 44 measurements and threshold 0.05

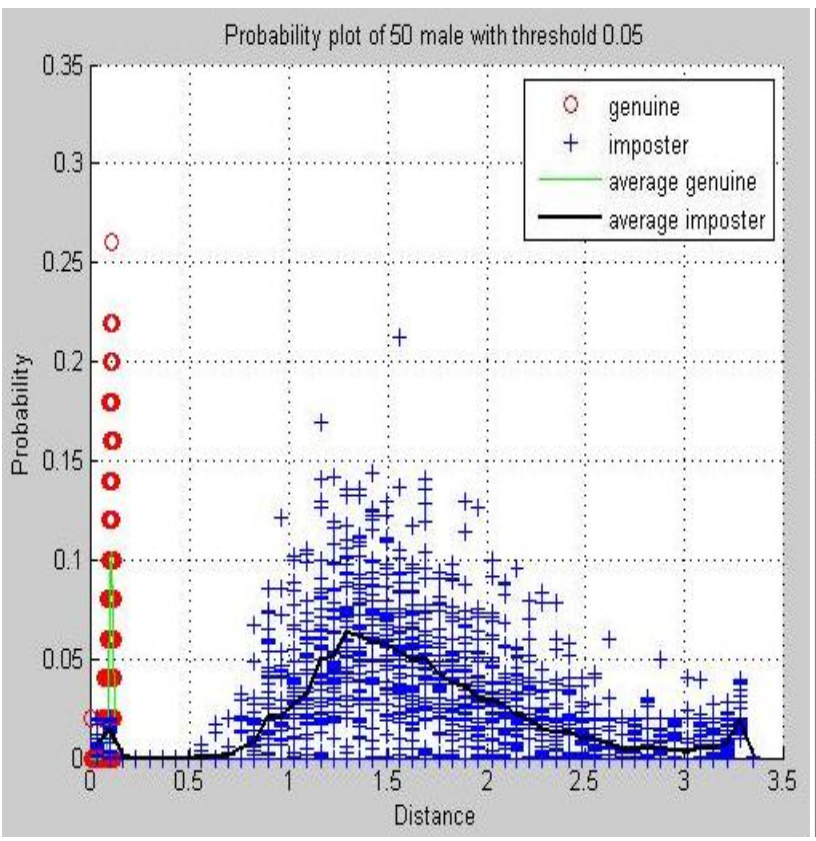

(c) 50 Male with 44 measurements and threshold 0.05

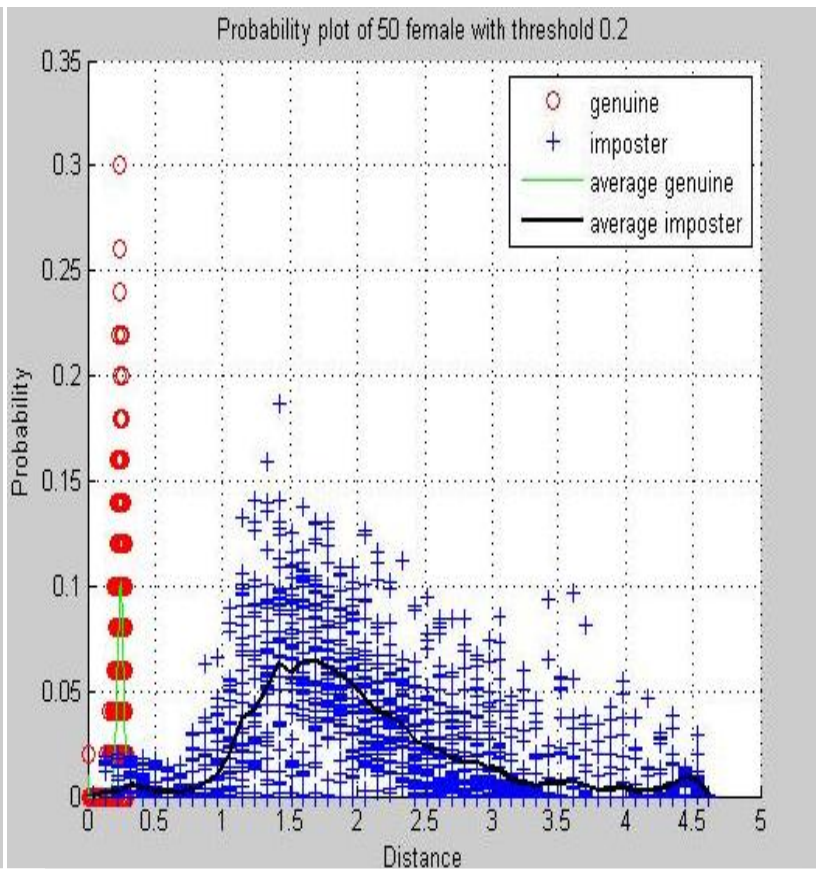

(b) 50 Female with 44 measurements and threshold 0.2

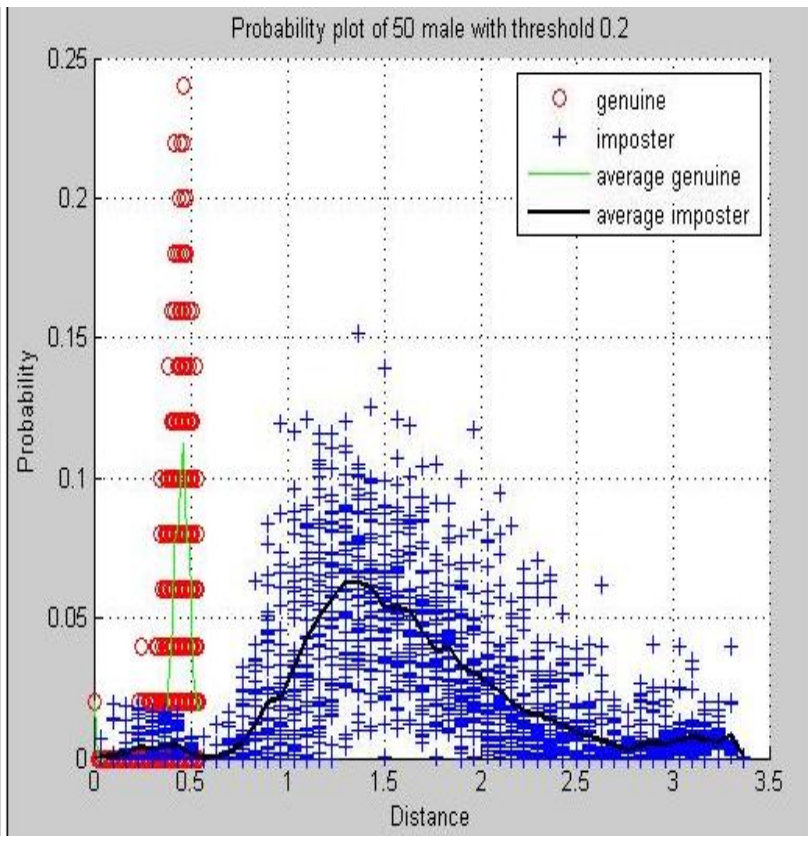

(d) 50 Male with 44 measurements and threshold 0.2 


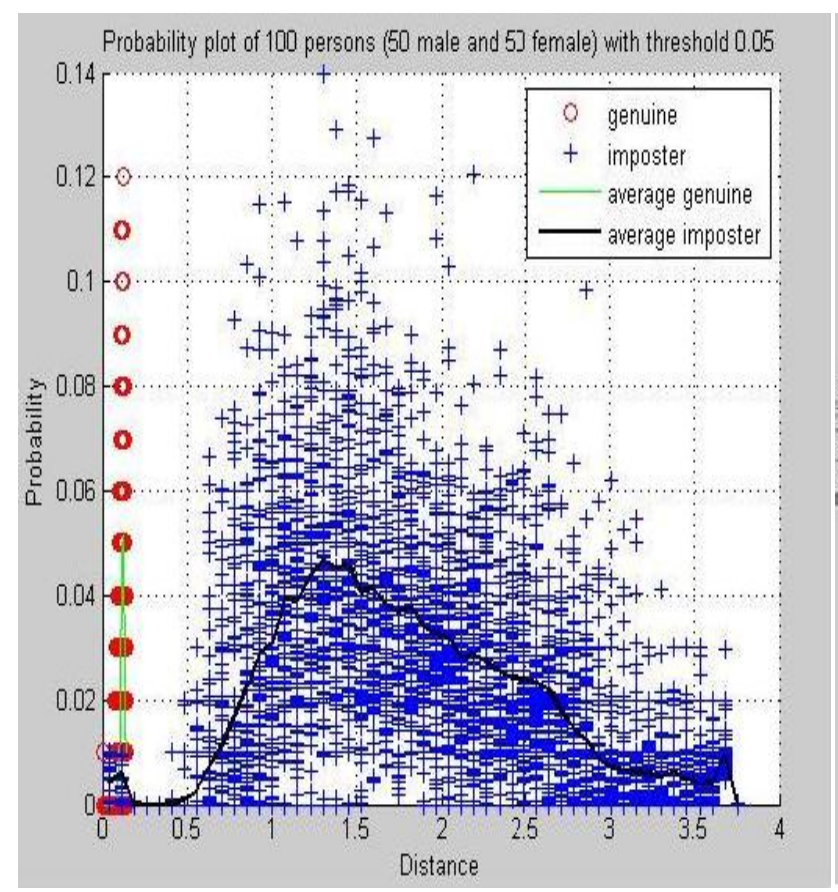

(e) 50 Male and 50 Female with 44 measurements and threshold 0.05

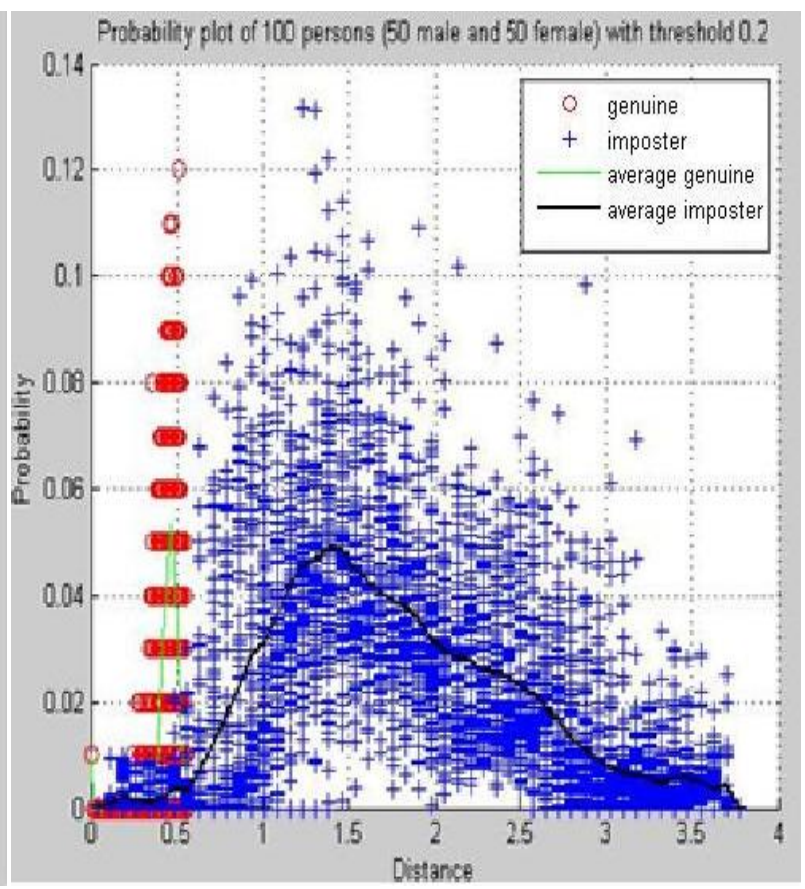

(f) 50 Male and 50 Female with 44 measurements and threshold 0.2

Figure 15: Probability of occurrence of Genuine and Imposters considering 44 measurements

We similarly get the scatter plots for 88 measurements in Figure 16 .

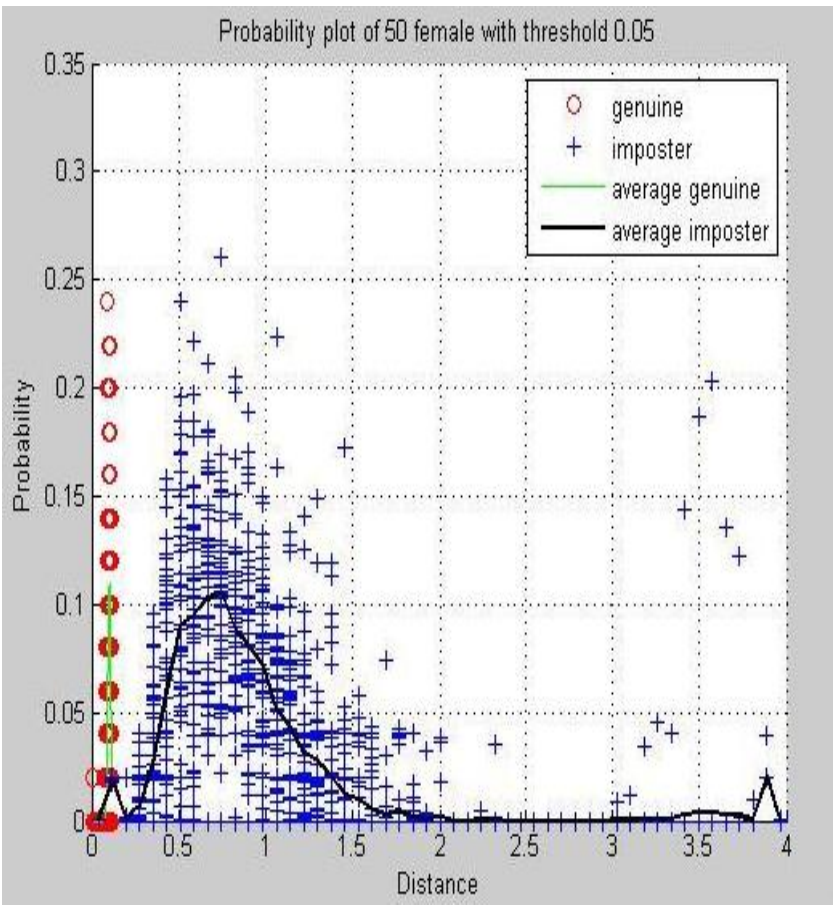

(a) 50 Female with 88 measurements and threshold 0.05

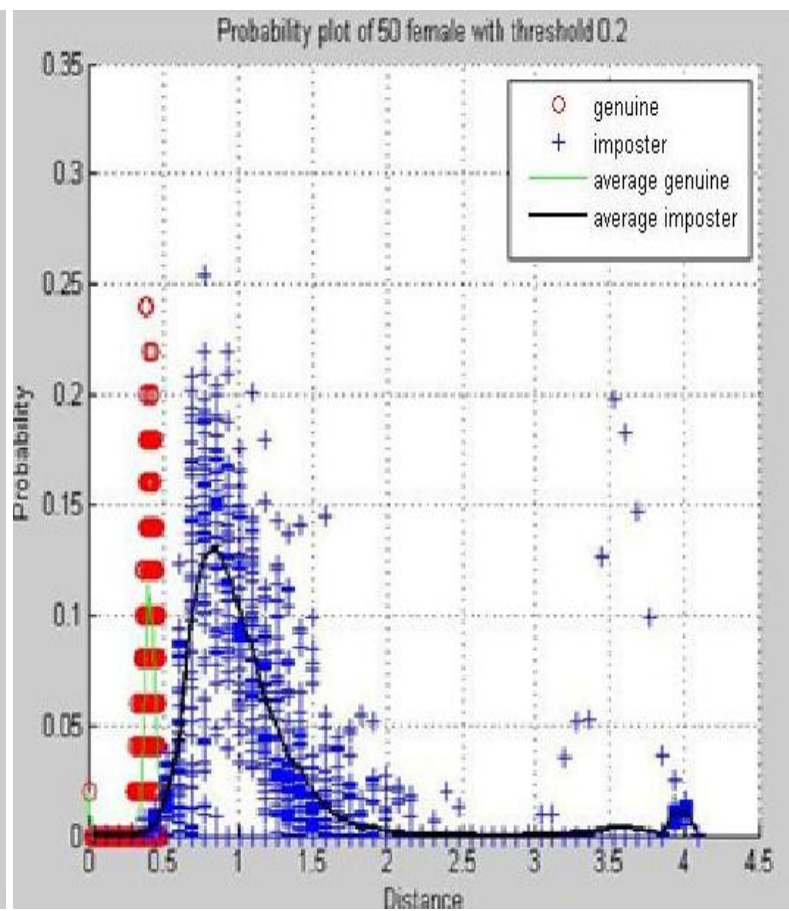

(b) 50 Female with 88 measurements and threshold 0.2 


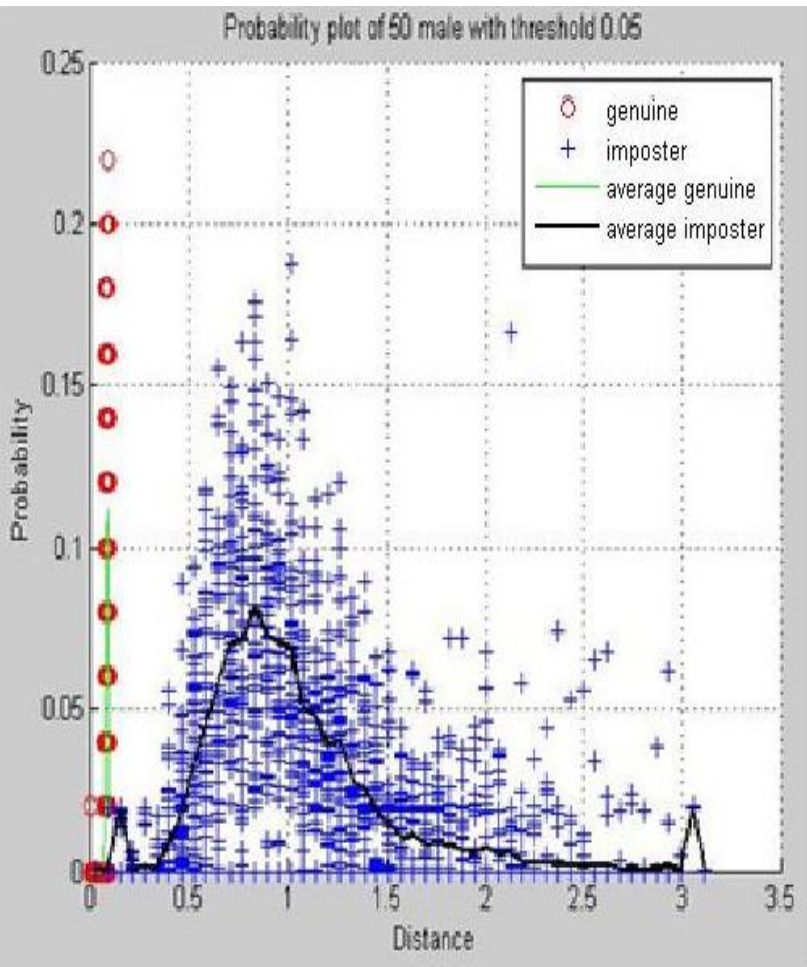

(c) 50 Male with 88 measurements and threshold 0.05

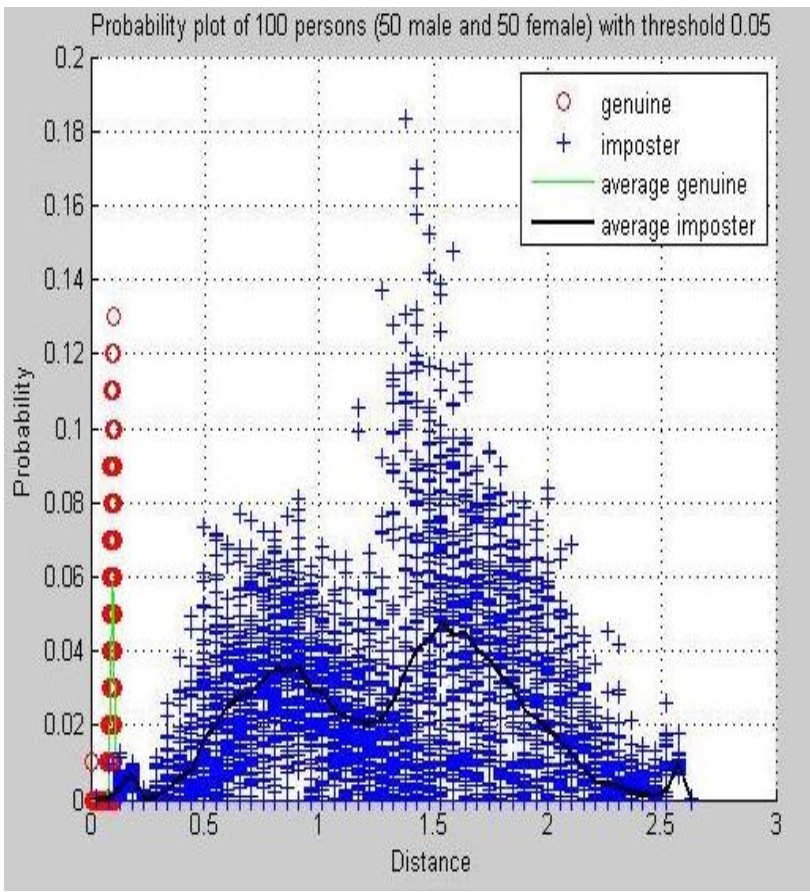

(e) 50 Male and 50 Female with 88 measurements and threshold 0.05

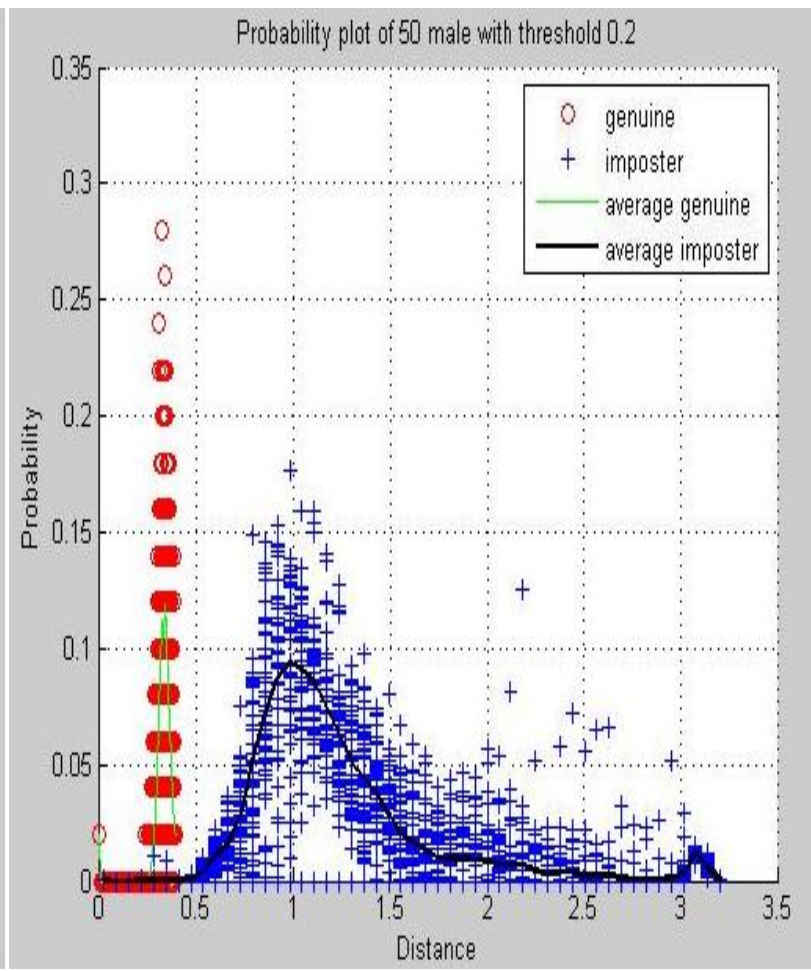

(d) 50 Male with 88 measurements and threshold 0.2

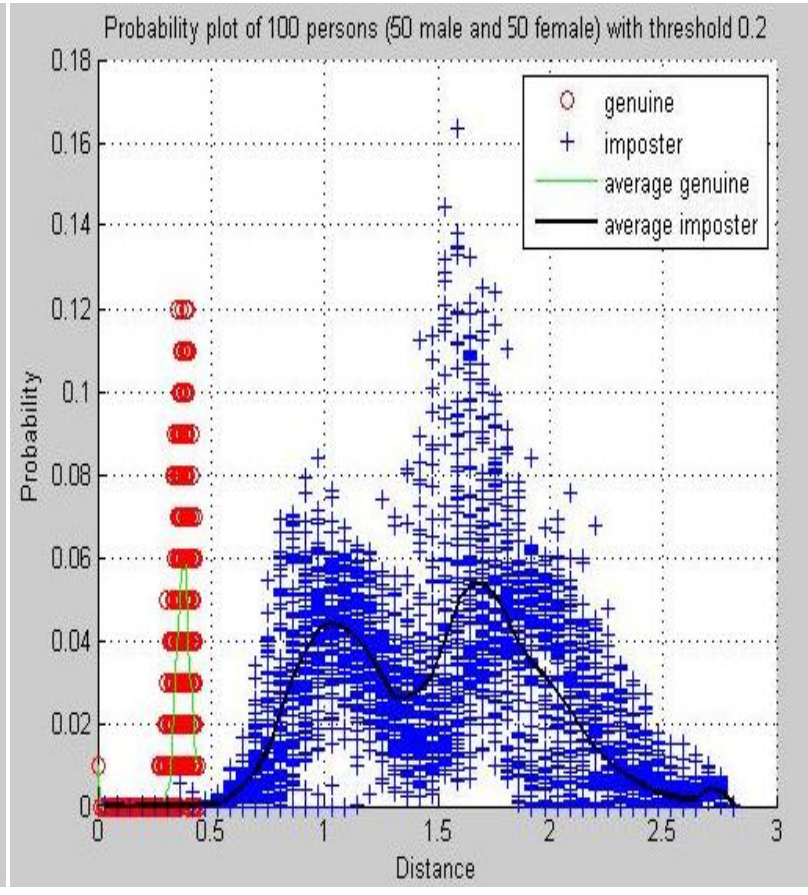

(f) 50 Male and 50 Female with 88 measurements and threshold 0.2

Figure 16: Probability of occurrence of Genuine and Imposters considering 88 measurements 
The above results are very helpful in classifying a subject as genuine or imposter person. For an unknown person or for a variation of known person, we can classify him/her as an imposter or genuine person based on where their distance falls from other people in the dataset. Also given the dataset and threshold, we can set the cutoff point which marks off genuine and imposters to the best level.

The distribution of data in the above curves is not normal. This can be observed in Figure 17, which shows the hypothesis test for normality. The normal probability plot is created with "normplot" function in MATLAB. It combines normal probability plots with hypothesis tests for normality. The plus signs plot the empirical probability versus the data value for each point in the data. The $y$-axis values are probabilities from zero to one, but the scale is not linear. In a normal probability plot, if all the data points fall near the line, an assumption of normality is reasonable. Otherwise, the points will curve away from the line, as shown in this figure.

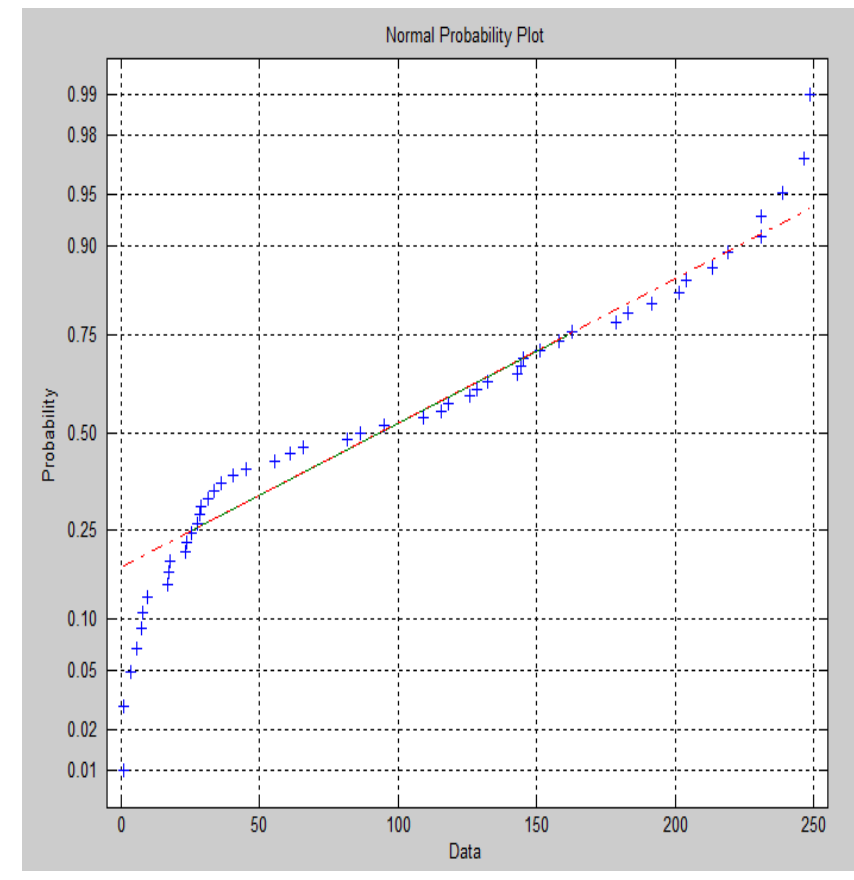

Figure 17: Normal probability plot of 50 male, 50 female and threshold 0.2

\subsubsection{Factor of age and gender}

We also plot the frequency-distribution plots related to age and gender as discussed in Section 5.5.2.1. The different age groups are: (i) Age group 1 - (18 yrs to $25 \mathrm{yrs}$ ), (ii) Age group 2 - (26 yrs to $40 \mathrm{yrs}$ ), (iii) Age group 3 - (41 yrs to $59 \mathrm{yrs}$ ), and (iv) Age group 4 (above $60 \mathrm{yrs}$ ). We also consider male and female genders in each age group. The results are shown in Figure 18 which shows the genuine-imposter probability curves. 

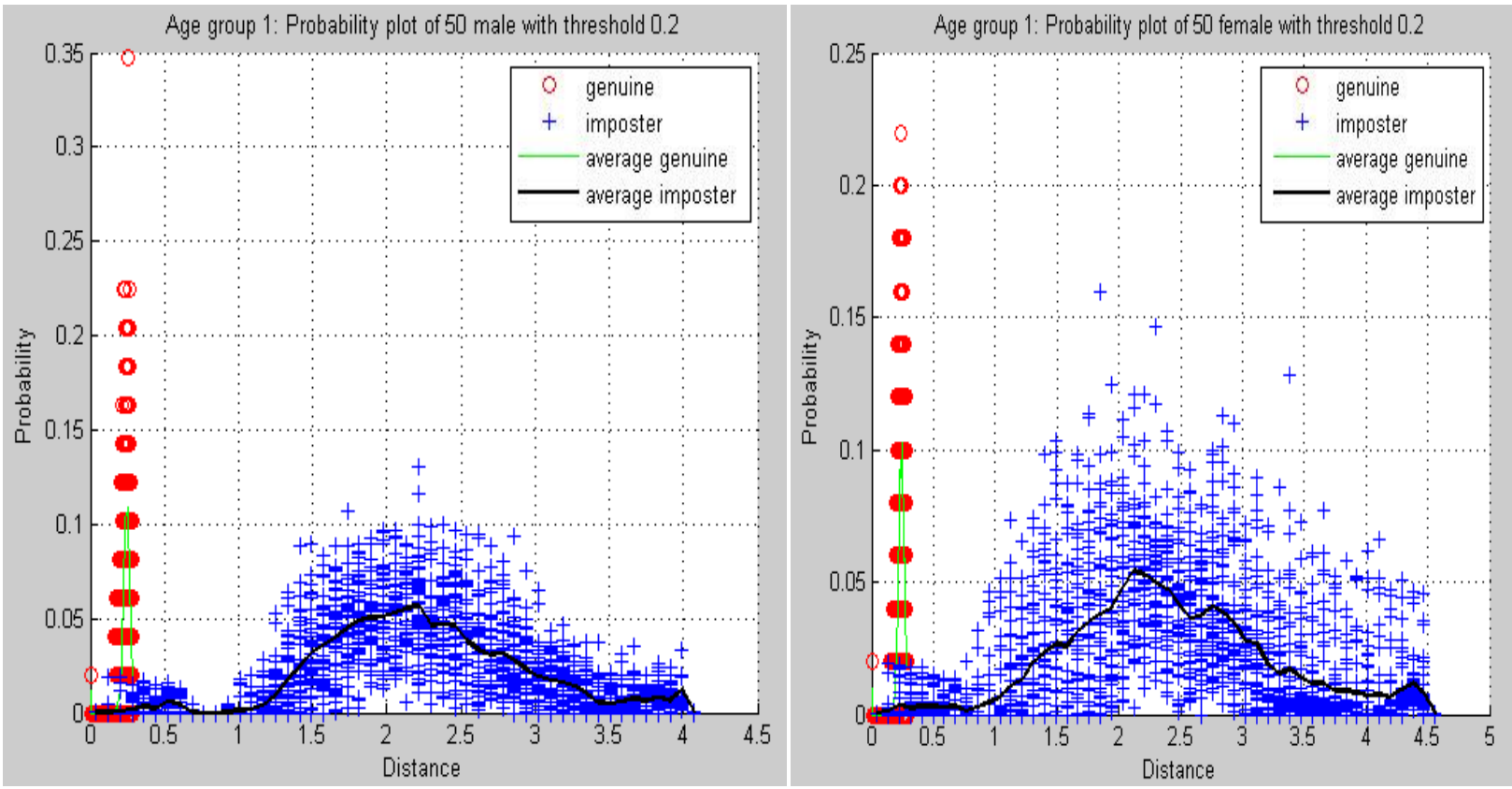

(a) Age group (i) - (18 yrs to $25 \mathrm{yrs}$ )
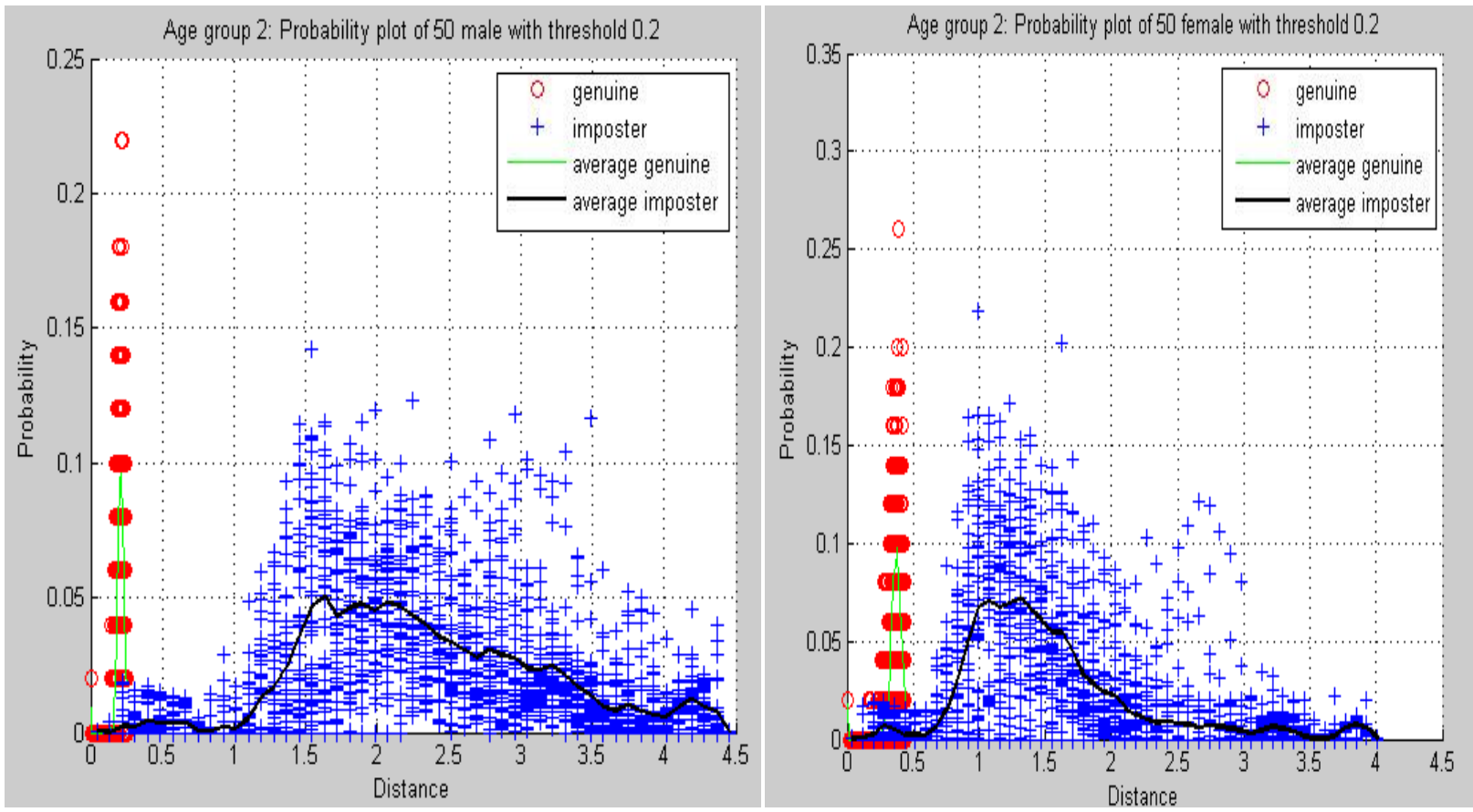

(b) Age group (ii) - (26 yrs to 40 yrs) 

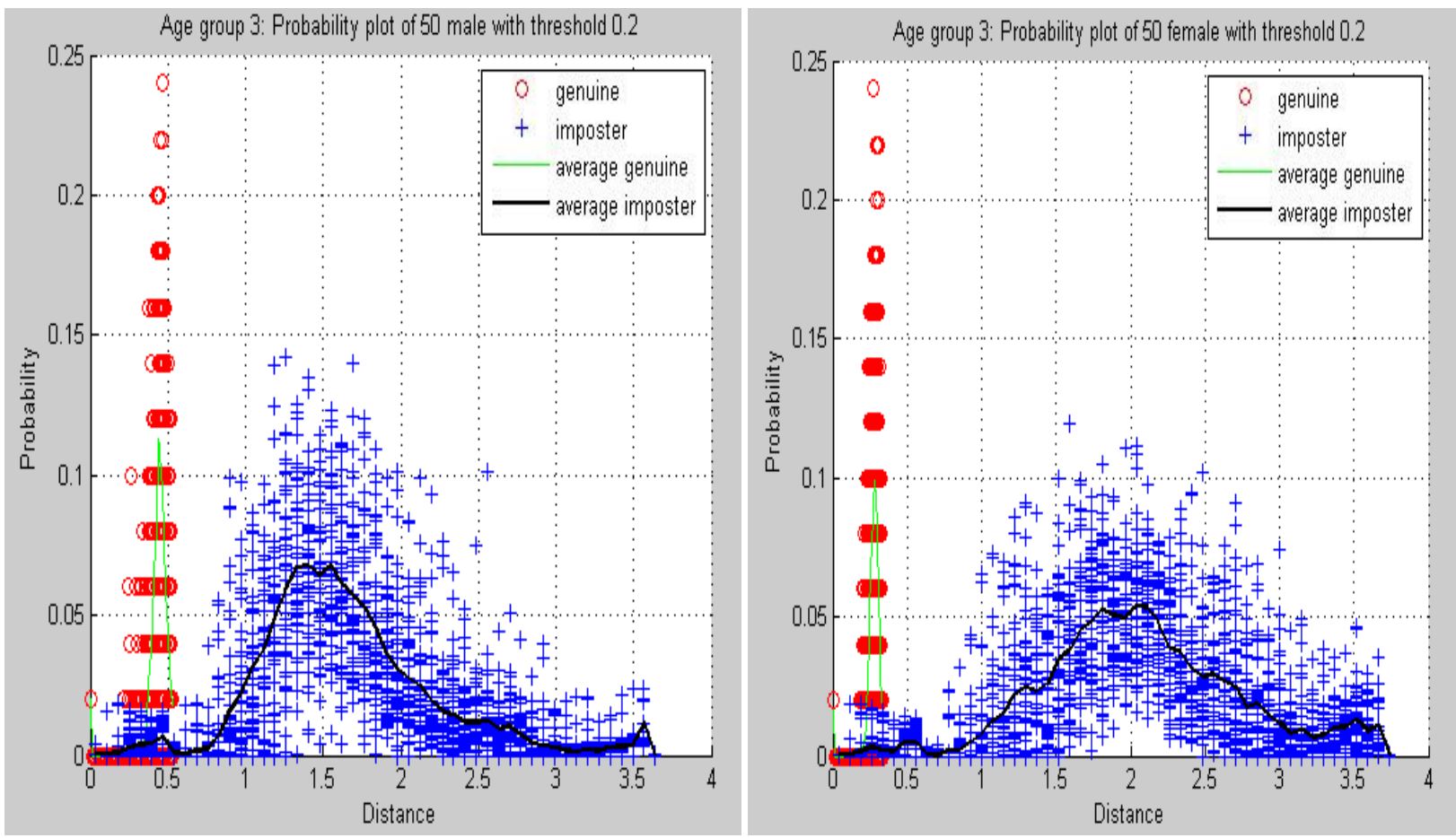

(c) Age group (iii) - (41 yrs to $59 \mathrm{yrs}$ )
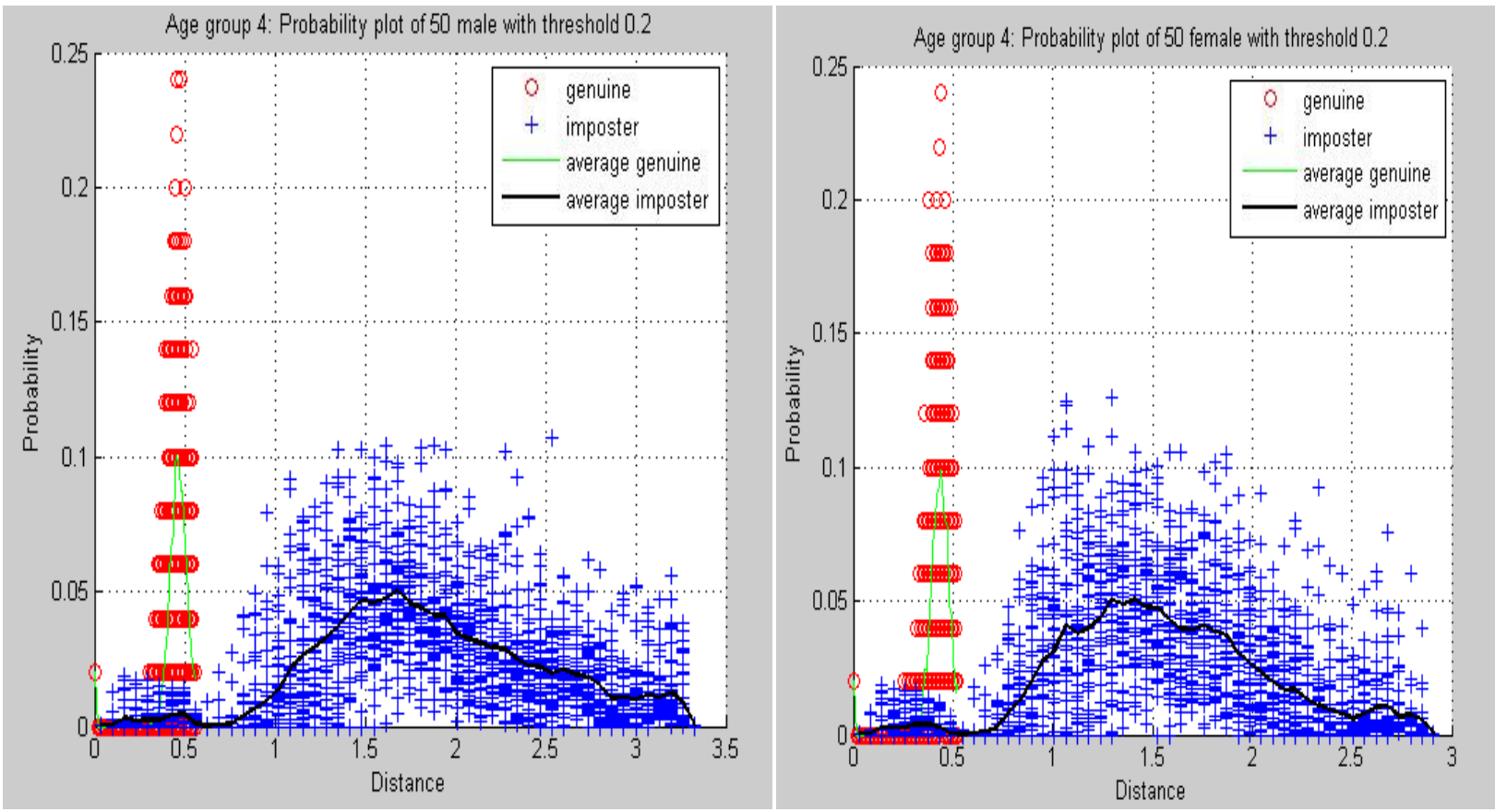

(d) Age group (iv) - (above 60 yrs)

Figure 18: Probability of occurrence of Genuine and Imposters in each age group considering male and female genders 
From these plots, we can decide the threshold at which a genuine person occurs in a group of imposters in each age group. Probability of occurrence of an imposter is more in female gender compared to male gender. Also genuine and imposter frequency is more in combined genders when compared to genders as a single group.

In Figure 16 (e) and (f), we get a bimodal graph. This can be reasoned to having both male and female genders as single set in computing the classification rates, rather than as individual groups.

\subsubsection{Clustering}

Using $K$-means and Agglomerative clustering, we get the clusters for 44 and 88 measurements for a training set of 100 people. They are shown in Figure 19.

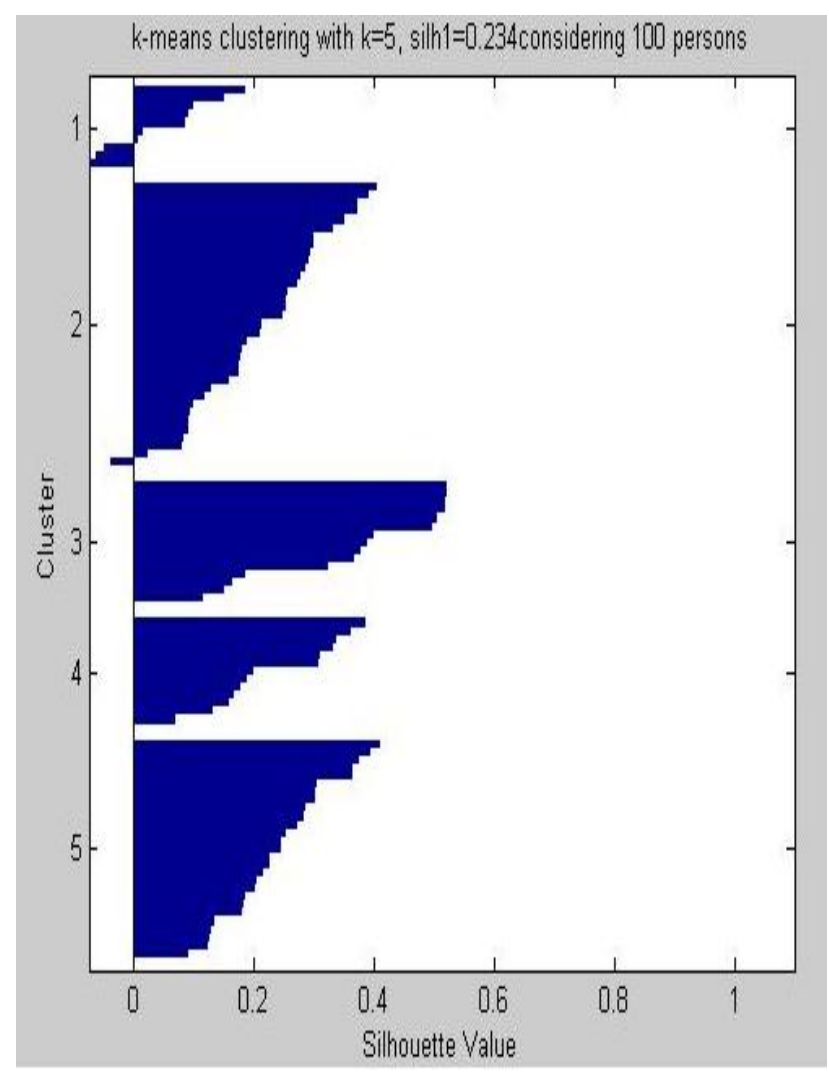

(a) $\mathrm{K}$-means clustering of 100 people considering 88 measurements

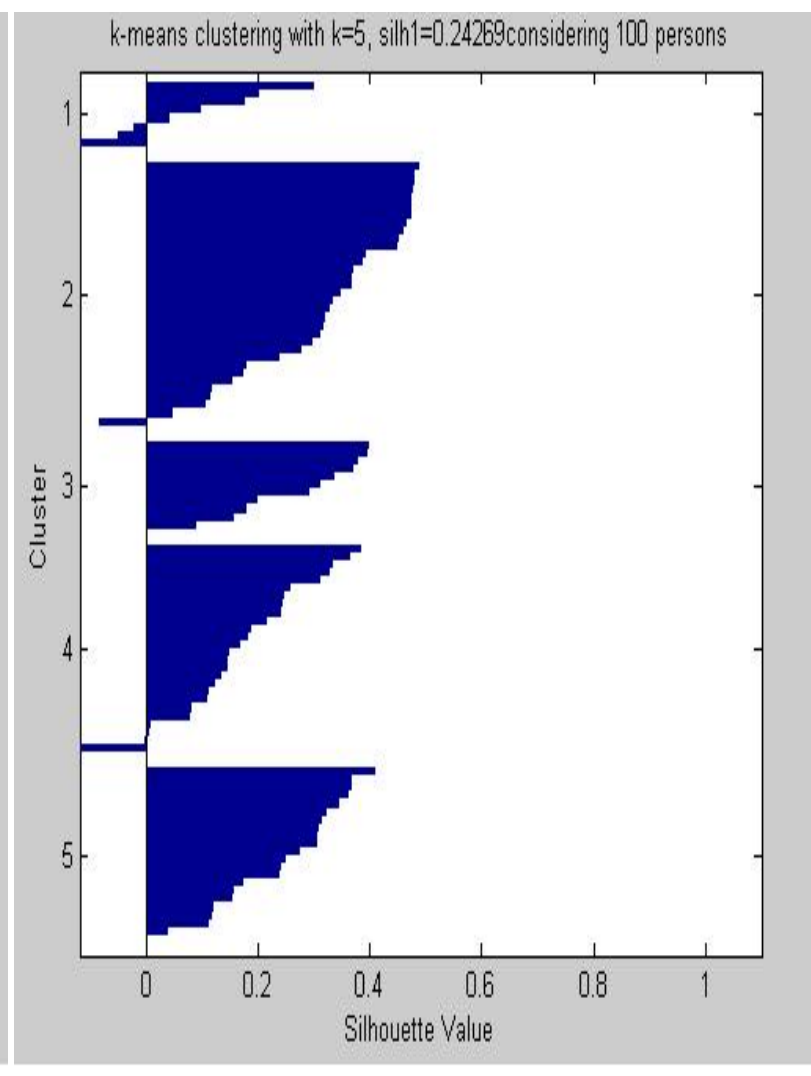

(b) $\mathrm{K}$-means clustering of 100 people considering 44 measurements 


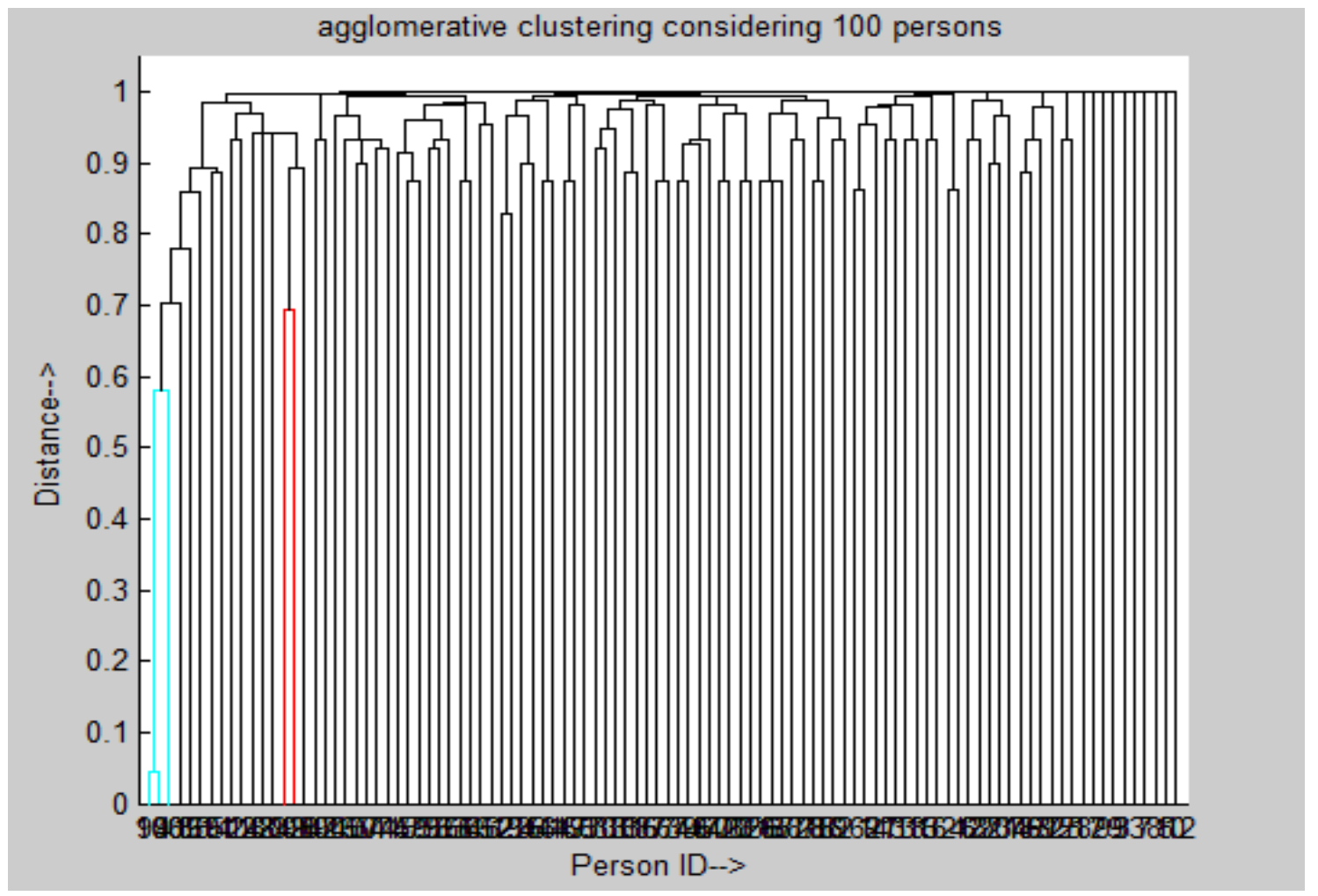

(c) Agglomerative clustering of 100 people considering 88 measurements

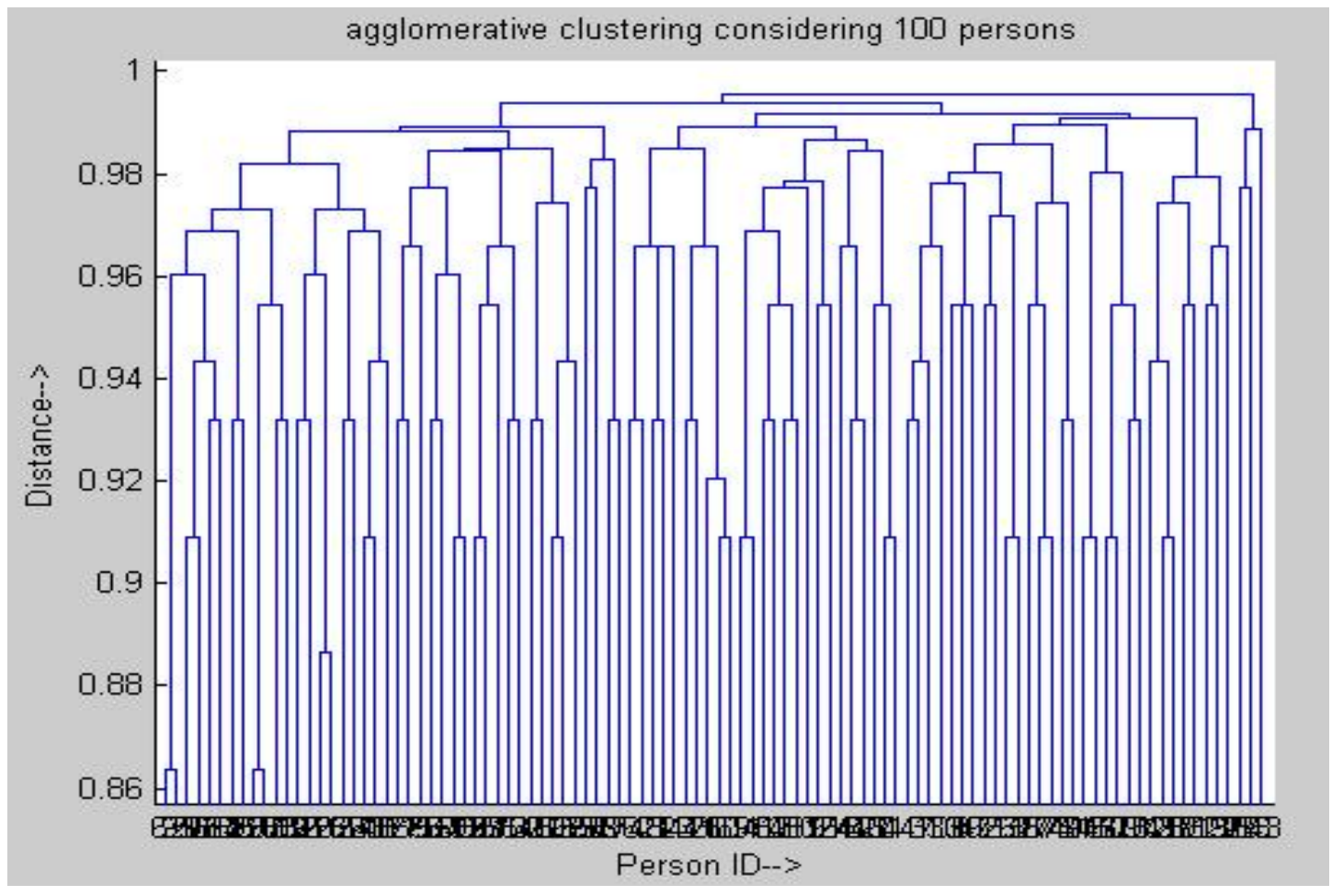

(d) Agglomerative clustering of 100 people considering 44 measurements

Figure 19: Clustering of a training set of 100 people considering 44 and 88 measurements 
From the above results, we can observe that Agglomerative clustering is more informative compared to K-means clustering. In Figure 19(c) and (d), if we take some threshold distance of, say, 0.98 , we can know how many persons are clustered above that threshold by marking a cutoff line. All persons above that line are clustered at that threshold.

\subsection{Discussion}

From the above results, we get to know the performance of CAESAR dataset. We initially selected 11 measurements (SET-11), and later did feature extraction on these measurements as mentioned in Section 4.5. We later consider 44 measurements (SET-44), and impart 50 variations on each of the original persons in both these sets while varying a threshold.

On each of these features, we carry out performance measures resulting in characteristic plots. From the initial figure of scatter plot, we get an idea about the relation between selected measurements. Later we generate the histogram over the complete dataset to know the distribution of these features over the entire population.

We later compute distance plots for both set of measurements. It can be observed that as the number of features considered increase from 11 to 44, the person-to-person distance increases. From Table 9 it can be detected that the minimum distance threshold increases from 0.5251 to 0.9116 for the complete set when the features increase from 11 to 44 respectively. Hence the persons with 44 features are better separated than the persons considered with just 11 features. Moving further to the classification rate plots, we defined classification rule as number of dissimilar persons whose Euclidean distance is greater than some threshold (Equation (5)). Based on this, we plotted the classification plots for 44 features and 88 features. The plots of classification rate of eigen Person, median Person, and average Person for both male and female groups are shown in each of the varying cases 1,2 and 3. At any given threshold, the classification rate is better for women than for men. From the results, we can observe that euclidean distance yields the higher classification rate compared to other distances. Also considering 44 features gives a better classification rate compared to 11 measurements and its extracted features. This can be attributed to the fact that the 11 features selected initially are not reliable in identifying people. Also we can say that metrology can distinguish people, by taking some threshold and below which if it finds just one person.

Hence distance plots and classification rate plots are helpful in distinguishing between persons. 
From the precision-recall plots, we note that practically the system is able to retrieve correctly the variations of original persons. We get to know about the accuracy of measurement in identifying persons. It can be observed from the results that considering 44 measurements performs better than 88 features.

The genuine acceptance rate and false acceptance rate is a ROC curve showing the performance of the system in retrieving genuine persons even with variations. We can observe that ROC curve is good enough in detecting a varied person in the dataset. For the 11 measurements and its variations, we see that features extracted through PCA perform better compared to other extracted features or the 11 original features.

We later plotted the frequency plots for genuine and imposters which is practically important. From these plots, we can decide on the threshold that can be used in marking off between imposters and genuine persons. We can know the Type-I and Type-II errors which are critical in performance of a biometric system. At some distance, we come across some imposters acting as genuine persons. But we can identify them because they are within some distance threshold.

Next the cluster plots are shown, using $K$-means and agglomerative clustering. Agglomerative clustering shows the distance threshold at which a particular number of persons have been clustered. If we select a threshold at some distance, the portion above this line will convey useful information for future study. 


\section{Chapter 6: Conclusion}

There are different recognition methods for human recognition. All the methods have their advantages and disadvantages. The major disadvantage of these recognition schemes is the human under study should be within the proximity of measuring instrument. But in practice, this may not be the case always. In such a situation we can make use of video metrology.

The main objective of this work has been to evaluate the performance of human metrology in distinguishing between persons using the CAESAR dataset. We can conclude that metrology can be used in distinguishing one person from other persons at a particular threshold. This can be observed from the distance plots, classification rate, precision-recall plots, ROC curves, and clustering methods.

Also feature extraction methods are used as described in this thesis. It can be said that PCA based features produce better results compared to other feature extraction methods or original measurements. This is observed from genuine acceptance rate and false acceptance rate plots.

Human metrology is fair enough in retrieving an original person from a pool of other original people and their variations. We can identify a genuine person from imposters.

Based on the results generated for training set and the complete set, we can say that human metrology is good in discriminating between persons, but care has to be taken to ensure that the parameters (like variation threshold, number of variations, or the distance threshold) are within some bounds.

From this thesis, we can say that Human metrology can be used in Biometrics to distinguish, identify and verify humans.

\section{Future Directions}

Here we discuss some future directions which can be carried out related to this work.

The performance measurement procedure discussed in this thesis can be used on real video to distinguish between humans. Also a larger training set can be employed and performance can be achieved.

This work can be extended to "allometric scaling", where the objective is to generate synthetic variations of human models or animals based on their shapes and sizes [14]. 


\section{References:}

[1] Feng Guo and Rama Chellappa, "Video Metrology Using a Single Camera," IEEE Transactions on pattern analysis and Machine Intelligence, Vol. 32, No. 7, July 20101329.

[2] http://store.sae.org/caesar/

[3] Jain, Anil K.; Flynn, Patrick; Ross, Arun A, Handbook of Biometrics, Springer 2006

[4] Electronic Privacy Information Center. "Face Recognition." January 19, 2006.

[5] Anil K. Jain, Arun Ross, and Salil Prabhakar, "An Introduction to Biometric Recognition."

[6] Akita, K. 1984, "Image sequence analysis of real world human motion", Pattern Recognition, 17(1):73-83, 1984

[7] Josef Bigün, Gérard Chollet, Gunilla Borgefors, "Book Audio-and video-based biometric person authentication," First International conference, AVBPA '97.

[8] Brett Allen, Brian Curless, Zoran Popovic, "Exploring the space of human body shapes: Data-driven synthesis under anthropometric control," Proceedings of the SAE Digital Human Modeling for Design and Engineering Conference, Rochester, MI. June 15-17, 2004.

[9] Brett Allen, Brian Curless, Zoran Popovic, "The Space of human body shapes: reconstruction and parameterization from range scans," ACM Transactions on Graphics (ACM SIGGRAPH 2003), 22, 3, 587-594.2003.

[10] Afzal Godil, Patrick Grother, Sandy Ressler "Human Identification from Body Shape," pp.386, Fourth International Conference on 3-D Digital Imaging and Modeling (3DIM '03), 2003.

[11] United Stated Air Force Research Laboratory, Civilian American and European Surface Anthropometry Resource (CAESAR), Final Report Volume II: Demographic and Measurement Descriptions, 2002. 
[12] R. Lymann Ott, Michael Longnecker, An Introduction to Statistical Methods and Data Analysis, $6^{\text {th }}$ Ed., Cengage Learning, 2010.

[13] Don Adjeroh, Deng Cao, Marco Piccirilli, and Arun Ross, "Predictability and Correlation in Human Metrology".

[14] T. McGraw, T. Kawai, J. Richards, "Allometric Scaling for Character Design", Computer Graphics Forum, 2010.

[15] Afzal Godil, Sandy Ressler "Retrieval and clustering from a 3D Human Database based on Body and Head Shape," in proceedings of 2006 Digital Human Modeling for Designand Engineering, July 2006, Lyon France.

[16] Christopher M. Bishop, Pattern Recognition and Machine Learning, Springer 2006.

[17] P. Jonathon Phillips, Patrick Grother, and Ross Micheals "Evaluation Methods in Face Recognition," Handbook of face recognition, Pages 328-348, 2005.

[18] Chorkin Chan and Pak-bong Wong, "A Branch and Bound Decision Tree Bayes Classifier for Robust Multi-font Printed Chinese Character Recognition," 11th - 13th November, 1992 Melbourne, Australia.

[19] C. Madden, M. Piccardi, "Height Measurement as a session-based Biometric for people matching across disjoint camera views," Image and vision computing New Zealand, pp. 282-286, 2005.

[20] D. Decarlo, D. Metaxas, M. Stone "An Anthropometric face model using variation techniques," Proceedings of ACM SIGGRAPH 98, ACM Press, Computer Graphics Proceedings, Annual Conference Series, 67-74. 1998.

[21] Hye-won Seo and Nadia M. Thalmann, "An automatic modeling of human bodies from sizing parameters" Proceedings of the 2003 Symposium on Interactive 3D Graphics, ACM Press, 19-26. 2003. 
[22] S. I. R. Ellison, T. Fearn, "Characterizing the performance of qualitative analytical methods: Statistics and terminology," TrAC, Trends in analytical chemistry vol. 24, pp. 468-476, 2005.

[23] Peter Somol, Pavel Pudil, Josef Kittler, "Fast Branch \& Bound Algorithms for Optimal Feature Selection," IEEE Transactions on Pattern Analysis and Machine Intelligence, vol. 26, no. 7, pp. 900-912, July 2004.

[24] Richard O. Duda, Peter E. Hart, David G. Stork, Pattern Classification, $2^{\text {nd }}$ Ed., Wiley Interscience, 2000. 